\title{
ESCR Abstracts 2021
}

Published online: 15 October 2021

(c) Springer Nature B.V. 2021

\section{1}

\section{Cardiovascular magnetic resonance imaging features and prognostic value in immune checkpoint inhibitor-induced myocarditis}

Farah Cadour, Jennifer Cautela, Stanislas Rapacchi, Arthur Varoquaux, Paul Habert, ARNAUD, Alexis Jacquier, Alexandra Meilhac, Franck Paganelli, Ugo Scemama, Franck Thuny

Keywords myocarditis, cardio-oncology, cardiac magnetic resonance, immune checkpoint inhibitor

Topic Area of Interest

Purpose/Objectives ICI-M is an emerging and severe complication of cancer treatment, for which diagnosis and risk stratification are challenging. We sought to analyze the cardiovascular magnetic resonance imaging (CMR) findings of immune checkpoint inhibitor (ICI)-induced myocarditis (ICI-M) and to explore their prognostic value.

Methods and Materials In this case-control multicenter study, the clinical, biological, and CMR data (including T1/ T2 map values) of adults with ICI-M $(\mathrm{n}=33)$ were compared with those of two other groups, including cancer patients without myocarditis scheduled to receive ICIs (w/o-M, $\mathrm{n}=21$ ) and patients with non-ICI-induced myocarditis (NI$\mathrm{M}, \mathrm{n}=85$ ). As secondary objective, we performed an exploratory analysis of the potential CMR predictors of major adverse cardiovascular events (MACE) in ICI-M patients. Results Compared with w/o-M, the global T2, native T1, and extracellular volume (ECV) z-scores were significantly higher in ICI-M (respectively $\mathrm{p}=0.004, \mathrm{p}<0.001$, and $\mathrm{p}=0.03$ for ECV). Late gadolinium enhancement (LGE) was more frequently observed in ICI-M than in w/o-M (82\% vs. $9 \%, \mathrm{p}<0.001$ ). The sensitivity of the main 2018-Lake Louise criteria was only $61 \%$ and the specificity was $100 \%$ in the diagnosis of ICI-M. No significant difference was found between ICI-M and NI-M in terms of the global native T1, global ECV, and global T2 z-scores. LGE was less frequent in ICI-M ( $82 \%$ vs. $100 \%, \mathrm{p}<0.001)$ but was more likely to involve the midwall layer $(\mathrm{p}<0.001)$ and septal segments $(\mathrm{p}<0.001)$ than in NI-M. For ICI-M patients, septal LGE was the only CMR predictor of MACE after adjustment for age, sex, risk factors, left ventricular ejection fraction $<50 \%$, and magnitude of increase in peak troponin I or $\mathrm{T}$ (adjusted HR, 3.57 [1.26-10.18]; $p=0.02$ ).

Conclusion ICI-M demonstrates specific features on CMR, and septal LGE may be a predictor of poor prognosis.

\section{References}

Mahmood SS., Fradley MG., Cohen JV., et al. Myocarditis in Patients Treated With Immune Checkpoint Inhibitors. J Am Coll Cardiol 2018;71:1755-64.

Zhang L., Zlotoff DA., Awadalla M., et al. Major Adverse Cardiovascular Events and the Timing and Dose of Corticosteroids in Immune Checkpoint Inhibitor-Associated Myocarditis. Circulation 2020;141:2031-4.

Ferreira VM., Schulz-Menger J., Holmvang G., et al. Cardiovascular Magnetic Resonance in Nonischemic Myocardial Inflammation: Expert Recommendations. J Am Coll Cardiol 2018;72:3158-76.

Zhang L., Awadalla M., Mahmood SS., et al. Cardiovascular magnetic resonance in immune checkpoint inhibitor-associated myocarditis. Eur Heart J 2020;41:1733-43.

Thavendiranathan P., Zhang L., Zafar A., et al. Myocardial T1 and T2 Mapping by Magnetic Resonance in Patients With Immune Checkpoint Inhibitor-Associated Myocarditis. J Am Coll Cardiol 2021;77:1503-16.

Bonaca MP., Olenchock BA., Salem J-E., et al. Myocarditis in the Setting of Cancer Therapeutics: Proposed Case Definitions for Emerging Clinical Syndromes in Cardio-Oncology. Circulation 2019;140:80-91.

Presenter's first name and last name

Farah Cadour

Presenter's city and country

Marseille, FRANCE 
Presenter's email address

farah.cadour@hotmail.fr

0002

\section{Transforming a pre-existing MRI environment into an interventional cardiac MRI suite}

\section{Geertruida P. Bijvoet, Robert J. Holtackers, Jouke Smink, Tom Lloyd, Cristy L.M. van den Hombergh, Luuk J.B.M. Debie, Joachim Wildberger, Kevin Vernooy, Casper Mihl, Sevasti-Maria Chaldoupi}

Keywords Interventional CMR, CMR-guided ablation, CMR-guided electrophysiological procedure, iCMR

\section{Topic Procedure}

Purpose/Objectives To illustrate the practical and technical challenges along with the safety aspects when performing MRI-guided electrophysiological procedures in a pre-existing diagnostic MRI environment.

Methods and Materials A dedicated, well-trained multidisciplinary interventional cardiac MRI team (iCMR team), consisting of electrophysiologists, imaging cardiologists, radiologists, anaesthesiologists, MRI physicists, electrophysiological (EP) and MRI technicians, biomedical engineers, and medical instrumentation technologists is a prerequisite for a safe and feasible implementation of CMR-guided electrophysiological procedures (iCMR) in a pre-existing MRI environment. A formal dry run "mock-up" to address the entire spectrum of technical, logistic, and safety issues was performed prior to obtaining final approval of the Board of Directors.

Results With this process we showed feasibility of our workflow, safety protocol, and bailout procedures during iCMR outside the conventional EP lab. The practical aspects of performing iCMR procedures in a pre-existing MRI environment were addressed and solidified. Finally, the influence on neighbouring MRI scanners was evaluated, showing no interference.

Conclusion Transforming a pre-existing diagnostic MRI environment into an iCMR suite is feasible and safe. However, performing iCMR procedures outside the conventional fluoroscopic lab, poses challenges with technical, practical, and safety aspects that need to be addressed by a dedicated multi-disciplinary iCMR team.

\section{Image 1}

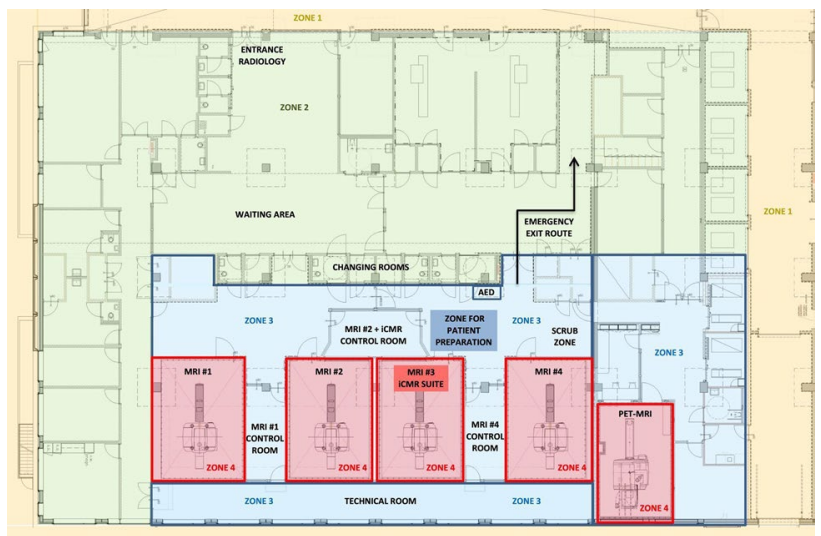

Image 2

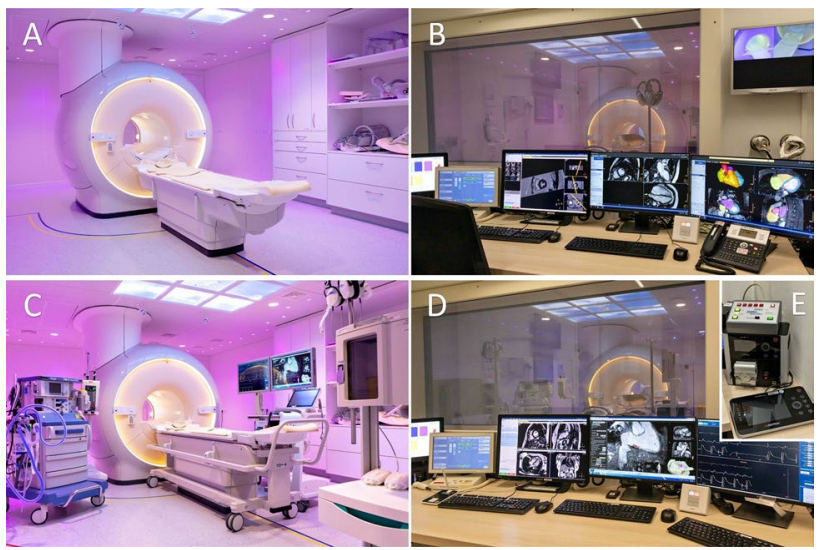

Presenter's first name and last name Geertruida P. Bijvoet

Presenter's city and country Maastricht, The Netherlands

Presenter's email address miranda.bijvoet@mumc.nl 


\section{3}

\section{Relationship between epicardial adipose tissue and diastolic function on routine cardiac magnetic resonance}

\section{Farah Cadour, Pierre Daudé, Patricia Ancel, Lauriane PINI, Franck Thuny, Anne Dutour, Monique Bernard, Alexis Jacquier, gaborit, Stanislas Rapacchi}

Keywords epicardial adipose tissue, cardiac magnetic resonance, diastolic function

\section{Topic Area of Interest}

Purpose/Objectives Purpose: Diastolic dysfunction (DD) is an often undetected condition in its early stages, although DD participates in heart failure, particularly in metabolic syndrome patients. In these patients, epicardial adipose tissue (EAT) overload has been associated to DD. Identifying concurrent biomarkers from routine clinics, including cardiac magnetic resonance (CMR), that could characterize patients with DD would help tailor care and prevent further cardiac degradation.

Objective This study sought to analyze if subjects with DD on CMR are dissociable based on routine CMR and clinical parameters.

Methods and Materials 141 adults with CMR were enrolled in this retrospective single-center study. Principal components analysis, hierarchical clustering and correlations on a vast array of demographical, biological, clinical variables, and CMR parameters (including DVR80 - proportion of diastole required to recover $80 \%$ stroke volume, and EAT surface) were conducted after stratification to adjust for age $(<50 / \geq 50$ years old $)$.

Results On the group $<50$ years old, unsupervised clustering distinguished three clusters of equivalent size. Cluster 3 had a "pathological phenotype" with obese (body mass index, BMI $40.31 \pm 7.77$ kg.m-2) and diabetes mellitus subjects presenting altered DVR80 and EAT overload. Cluster 2 and 1 differed only on sex predominance and diabetes mellitus distribution. EAT overload, BMI, and diabetes mellitus were significantly correlated to DVR80 (respectively $\mathrm{r}=0.28, \mathrm{p}=0.02 ; \mathrm{r}=0.40, \mathrm{p}<0.001 ; \mathrm{r}=0.38, \mathrm{p}=0.001)$. Neither significant association with DVR80 nor distinctive clusters were found in the group $\geq 50$ years old, suggesting a more complex multifactorial phenomenon.
Conclusion Diabetic and overweighted subjects under 50 years old undergoing CMR should be screened with particular attention for EAT overload and DVR80 alteration from routine cine-sequence to orient patient for additional investigations for DD diagnosis, even if asymptomatic.

\section{References}

Jeong E-M, Dudley SC. Diastolic Dysfunction. Circ J Off J Jpn Circ Soc. 2015;79(3):470-477. https://doi.org/10. 1253/circj.CJ-15-0064

Nakanishi K, Fukuda S, Tanaka A, Otsuka K, Taguchi H, Shimada K. Relationships Between Periventricular Epicardial Adipose Tissue Accumulation, Coronary Microcirculation, and Left Ventricular Diastolic Dysfunction. Can J Cardiol. 2017;33(11):1489-1497. https://doi.org/10. 1016/j.cjca.2017.08.001

Westenberg JJM. CMR for Assessment of Diastolic Function. Curr Cardiovasc Imaging Rep. 2011;4(2):149-158. https://doi.org/10.1007/s12410-011-9070-z

Kawaji K, Codella NCF, Prince MR, et al. Automated segmentation of routine clinical cardiac magnetic resonance imaging for assessment of left ventricular diastolic dysfunction. Circ Cardiovasc Imaging. 2009;2(6):476-484. https://doi.org/10.1161/CIRCIMAGING.109.879304 Mendoza DD, Codella NC, Wang Y, et al. Impact of diastolic dysfunction severity on global left ventricular volumetric filling — assessment by automated segmentation of routine cine cardiovascular magnetic resonance. J Cardiovasc Magn Reson. 2010;12(1):46. https://doi.org/10.1186/ 1532-429X-12-46

de Wit-Verheggen VHW, Altintas S, Spee RJM, et al. Pericardial fat and its influence on cardiac diastolic function. Cardiovasc Diabetol. 2020;19. https://doi.org/10.1186/ s12933-020-01097-2

Presenter's first name and last name

Farah Cadour

Presenter's city and country

Marseille, FRANCE

Presenter's email address

farah.cadour@hotmail.fr 


\section{4}

\section{Myocardial T2 mapping and correlations with clinical and CMR parameters in patients with systemic sclerosis}

\section{Alessia Pepe, Luna Gargani, Cosimo Bruni, Camilla Cavallaro, Marco Gobbo, Gennaro D'Angelo, Nicola Martini, Francesco Grigioni, Gianfranco Sinagra, Sophie Mavrogeni, Marco Matucci-Cerinic, Antonella Meloni}

Keywords Myocardium, MR/MR-Angiography, Tissue characterisation

\section{Topic Special Focus}

Purpose/Objectives Chronic myocardial inflammation plays a crucial role in the pathogenesis of cardiac involvement in systemic sclerosis (SSc) patients. T2 mapping cardiovascular magnetic resonance can noninvasively detect subtle forms of myocardial inflammation with high sensitivity and specificity. Our aim was to evaluate the prevalence of cardiac involvement by $\mathrm{T} 2$ mapping and its correlation with clinical and CMR parameters in SSc patients.

Methods and Materials Fifty-one consecutive SSc patients (mean age 51.8 \pm 13.7 years, 42 females) and 51 healthy subjects matched for age and gender underwent clinical, bio-humoral assessment, and CMR at 1.5 T (Signa Artist, GE Healthcare). The imaging protocol included: cine, $\mathrm{T} 2$ and $\mathrm{T} 1$ mapping by multi-echo fast-spin-echo and MOLLI sequences, respectively, late gadolinium enhancement (LGE), and STIR T2-weighted sequences. Native T2 and $\mathrm{T} 1$ values were assessed in all 16 myocardial segments and the global value was the mean.

Results Global myocardial T2 values were significantly higher in SSc patients than in healthy subjects (Fig. 1). In patients, global myocardial $\mathrm{T} 2$ values were significantly lower in males than in females $(55.24 \pm 4.78$ vs $58.19 \pm 3.63$; $\mathrm{P}=0.042)$ and not associated with age. Thirty-one $(60.8 \%)$ patients had an elevated global myocardial T2 value (ET2), according to our local cut-offs. Only 5 patients $(9.8 \%)$ showed myocardial edema by $\mathrm{T} 2$-weighted images (4 patients had also an ET2). T2 values were comparable between patients without and with edema by STIR. All 6 patients with diffuse cutaneous systemic sclerosis (dcSSc) showed an increased $\mathrm{T} 2$ value, making the frequency of this form significantly more frequent among patients with elevated versus normal $\mathrm{T} 2$ value $(19.4 \%$ vs $0.0 \% ; \mathrm{P}=0.036)$. Positive $\mathrm{STIR}$ images were comparable in pts with dcSSc and lcSSc. All other clinical/CMR parameters were comparable between patients with normal and elevated T2. Native T1 values were significantly higher in patients with ET2 (Fig. 2).

Conclusion More than half of patients with SSc showed an increased myocardial $\mathrm{T} 2$ value, associated with the presence of the diffuse cutaneous form and of diffuse myocardial fibrosis. CMR T2 mapping seems to be a more sensitive parameter for detecting edema and it should be considered for an early assessment of subclinical myocardial inflammation.

\section{Image 1}

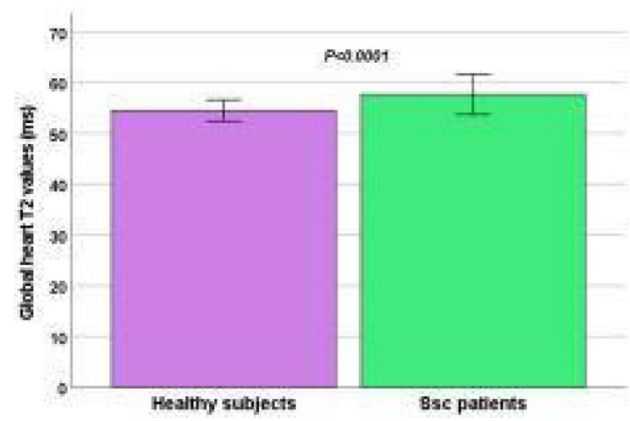

Image 2

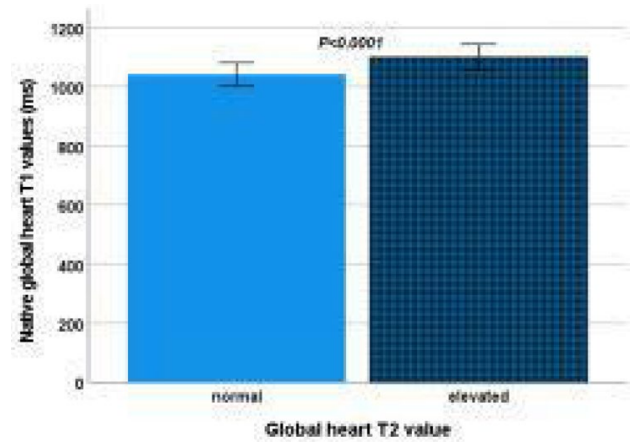

Presenter's first name and last name

Alessia Pepe

Presenter's city and country

Pisa, Italy

Presenter's email address alessia.pepe@ ftgm.it 
0005

\section{Impact of the COVID-19 pandemic in the MR availability for iron overloaded patients}

\section{Alessia Pepe, Laura Pistoia, Massimiliano Missere, Riccardo Righi, Antonino Vallone, Priscilla Fina, Stefania Renne, Ada Riva, Angelantonio Vitucci, Francesco Massei, F. Mehtap Pasin, Antonella Meloni}

Keywords Myocardium, MR/MR-Angiography, Tissue characterisation

\section{Topic Special Focus}

Purpose/Objectives The $\mathrm{T} 2 *$ magnetic resonance imaging (MRI) technique for the noninvasive quantification of iron overload has significantly improved the survival of patients with hemoglobinopathies by tailoring the chelation therapy. In Italy, the E-MIOT (Extension-Myocardial Iron Overload in Thalassemia) Network, a collaborative project among MRI and hematological centers, has assured high-quality quantification of iron in vital organs such as heart, liver, and pancreas. The COVID-19 pandemic has disrupted healthcare services around the world, also leading to postpone or delete deferable diagnostic evaluations. We evaluated the impact of the COVID-19 pandemic on MRI services for iron overload quantification in Italy.

Methods and Materials The activity of the MRI centers of the E-MIOT Network in the year 2020 was compared to the activity in the same months of 2019. A specific survey was filled out by the MRI operators to evaluate if the availability of MRI slots for patients with hemoglobinothies was reduced and the reasons.

Results In comparison with the 2019, in 2020 there was a significant reduction in the number of T2* MRIs performed(350 vs 656; $\mathrm{P}<0.0001)$. Specifically, there was a marked decline $(86.9 \%)$ in the four-month period MarchJune 2020, a reduction in the gap between the two years in the three-month period July-September, and a new decline (41.4\%) in the three-month period October-December (Fig. 1). Figure 2 shows the \%decline for each center (vertical axis $=0$ in absence of $\%$ decline). In the trimester July-September only Pisa and Taranto dropped the number of T2* MRIs, due to the rescheduling of the MRIs deleted during the lockdown. In the trimester October-December the reduction of the T2* MRIs at Ferrara and Lamezia was due to general reduction in the number of MRIs/day for the sanitation procedures. In the other centers the availability for T2* MRI scans was unchanged in comparison to 2019 , but the patients refused the MRI for fear of COVID.
Conclusion The COVID-19 pandemic is having a strong negative impact on the quantification of iron overload by MRI, which may seriously worsen the prognosis of patients with hemoglobinopathies. Strategies to ensure proved lifesaving MRI exam and to reassure patients about the health safety of the hospitals are recommended.

\section{Image 1}

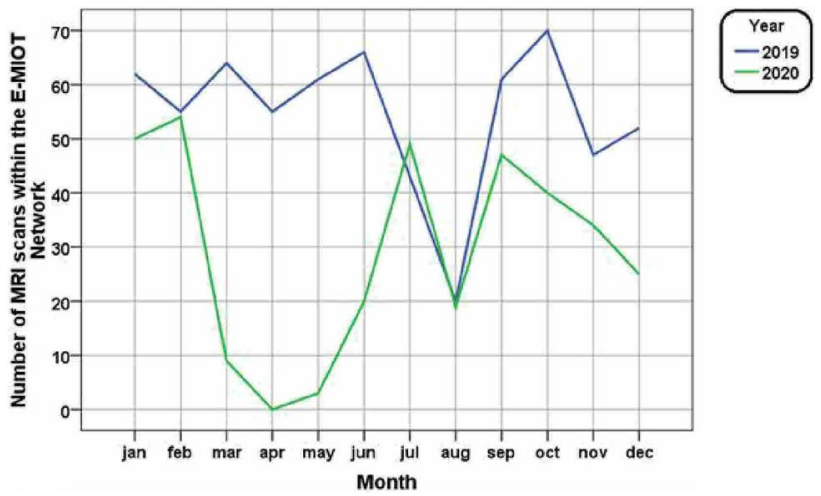

Image 2

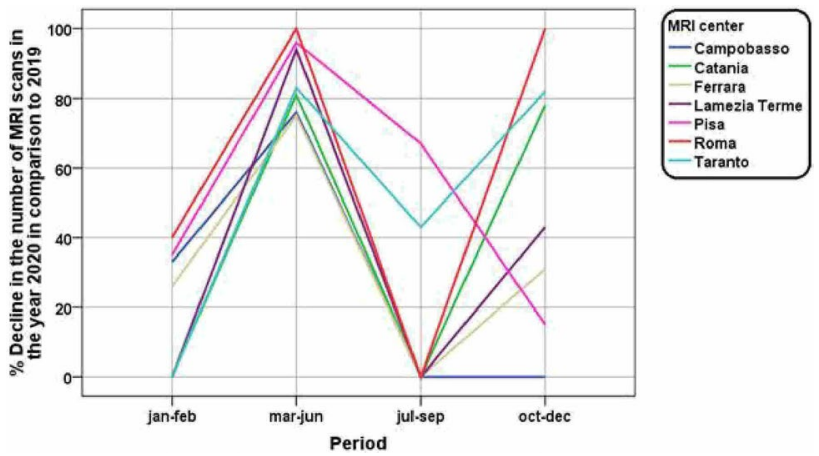

Presenter's first name and last name Alessia Pepe

Presenter's city and country Pisa, Italy

Presenter's email address alessia.pepe@ftgm.it 


\section{6}

\section{Multiparametric Cardiac Magnetic Resonance assessment in sickle $\beta$-thalassemia}

Alessia Pepe, Antonella Meloni, Giacomo Agliata, Gennaro Restaino, Giuseppe Peritore, Luigi Barbuto, Saveria Campisi, Sabrina Bagnato, Letizia Tedesco, Valentina Carrai, Vincenzo Positano, Laura Pistoia.

Keywords Myocardium, MR/MR-Angiography, Outcomes analysis, Tissue characterisation.

\section{Topic Special Focus.}

Purpose/Objectives Sickle $\beta$-thalassemia ( $\mathrm{S} \beta$-thal) is a hereditary hemoglobinopathy resulting from the combined heterozygosity for sickle cell and $\beta$-thalassemia genes. Cardiac involvement in $\mathrm{S} \beta$-thal patients has been poorly investigated. We aimed to evaluate myocardial iron overload and cardiac function by cardiovascular magnetic resonance (CMR) in patients with $S \beta$-thal.

Methods and Materials $111 \mathrm{~S} \beta$-thal patients consecutively enrolled in the Myocardial Iron Overload in Thalassemia (MIOT) network were studied and compared with 46 sickle cell disease (SCD) patients. Moreover, biatrial and biventricular function CMR parameters of $S \beta$-thal patients were compared with those of 111 healthy volunteers, matched by gender and age. Myocardial iron overload (MIO) was assessed by $\mathrm{T} 2 *$ technique. Cine images were acquired to quantify biventricular function. Macroscopic myocardial fibrosis was evaluated by late gadolinium enhancement (LGE) technique.

Results In S $\beta$-thal and SCD patients morphological and functional MR parameters were no significantly different, except for left atrial area and SVI $(p=0.023$ and $p=0.048$, respectively) that were significantly higher in SCD patients compared to $\mathrm{S} \beta$-thal. No significant differences between the two gropus were found in terms of myocardial iron overload and macroscopic myocardial fibrosis. When compared to healthy subjects, $S \beta$-thal patients showed significantly higher biatrial and biventricular parameters except for LVEF that was significantly lower (Fig. 1).

Conclusion The CMR analysis confirmed that $S \beta$-thal and SCD patients are phenotypically similar. Since $S \beta$-thal patients showed markedly different morphological and functional indices from healthy subjects, it would be useful to identify $S \beta$-thal/SCD-specific biatrial and biventricular reference values.
Image 1

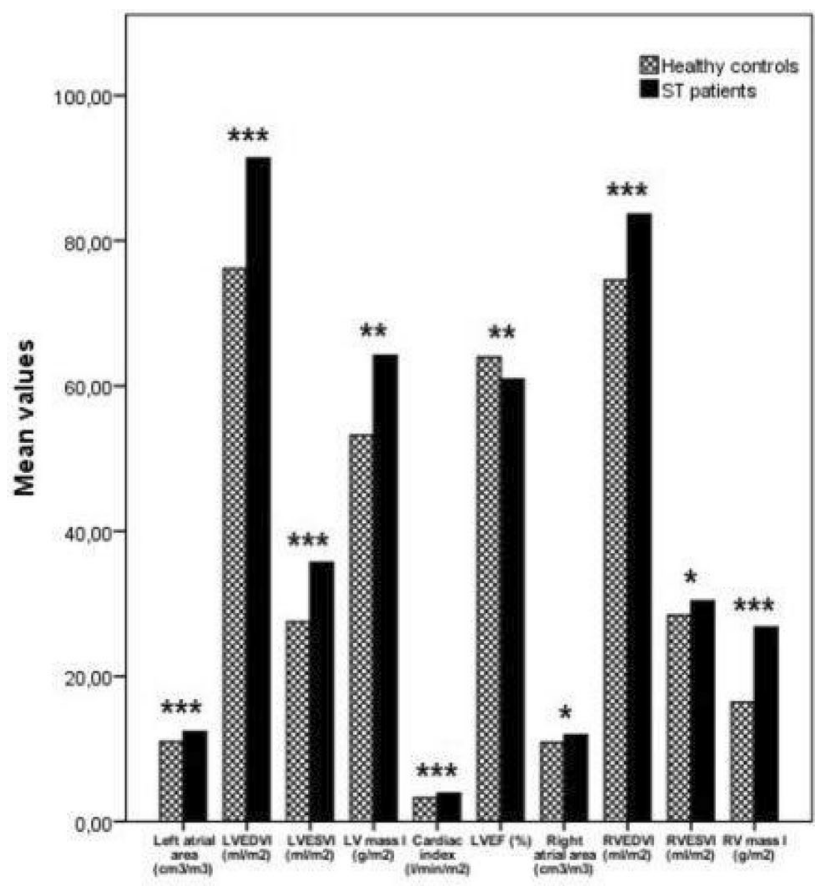

Presenter's first name and last name

Alessia Pepe

Presenter's city and country

Pisa, Italy

Presenter's email address

alessia.pepe@ftgm.it

\section{7}

\section{Myocardial T2 values by a segmental approach with healthy ageing and gender}

Alessia Pepe, Nicola Martini, Vincenzo Positano, Gennaro D'Angelo, Andrea Barison, Giancarlo Todiere, Chrysanthos Grigoratos, Petra Keilberg, Laura Pistoia, Luna Gargani, Andrea Ripoli, Antonella Meloni

Keywords Myocardium, MR/MR-Angiography, Tissue characterisation

Topic Special Focus 
Purpose/Objectives No data are available in literature about normal ranges for $\mathrm{T} 2$ in human myocardium using GE scanners.Our aims were to obtain myocardial regional and global T2 values as a reference for normality for the first time using a GE scanner and to assess their association with physiological variables.

Methods and Materials A stratified approach was adopted for healthy volunteers recruitment, ensuring the presence of 10 participants for both genders in each age decile: $20-30,30-40,40-50,50-60,60-70$ years. Basal, medium, and apical short-axis slices of the left ventricle were acquired by a multi-echo fast-spin-echo (MEFSE) sequence. Image analysis was performed with a commercially available software package. $\mathrm{T} 2$ value was assessed in all 16 myocardial segments and global value was the mean. Results The global T2 value averaged across all subjects was $52.2 \pm 2.5 \mathrm{~ms}$. Inter-study, intra-observer, and inter-observer reproducibility was good (coefficient of variation $<5 \%$ ). The $3.6 \%$ of segments was excluded because of artifacts and/or partial-volume effects. Segmental $\mathrm{T} 2$ values differed significantly $(\mathrm{P}<0.0001)$, with the lowest value in the basal anterolateral segment $(50.0 \pm 3.5 \mathrm{~ms})$ and the highest in the apical lateral segment $(54.9 \pm 5.1 \mathrm{~ms})$. Mean T2 was significantly lower in the basal slice compared to both medium $(51.0 \pm 2.4 \mathrm{vs}$ $51.8 \pm 2.6 \mathrm{~ms} ; \mathrm{P}<0.0001)$ and apical slices $(51.0 \pm 2.4 \mathrm{vs}$ $54.2 \pm 3.7 \mathrm{~ms} ; \mathrm{P}<0.0001)$, and in the medium slice than in the apical slice $(51.8 \pm 2.6$ vs $54.2 \pm 3.7 \mathrm{~ms} ; \mathrm{P}<0.0001)$. Aging was associated with increased segmental and global T2 values. Females showed higher T2 values than males. Mean T2 values, standard deviation, and lower/upper limits of normal for all 16 myocardial segments are shown in Fig. 1 for males and in Fig. 2 for females, considering separately each age group.

Conclusion The optimized MEFSE sequence allows for robust, reliable, and reproducible quantification of segmental T2 values. T2 values differ among myocardial slices and are influenced by age and gender, making mandatory to define gender- and age-specific segmental reference values for distinguishing between healthy and diseased myocardium. The normal ranges defined in this study on a large cohort of healthy subjects could be used as reference by other sites using the same sequence, allowing them to recruit a smaller population and accelerating the spread of myocardial T2 mapping in the clinical arena.

\section{Image 1}
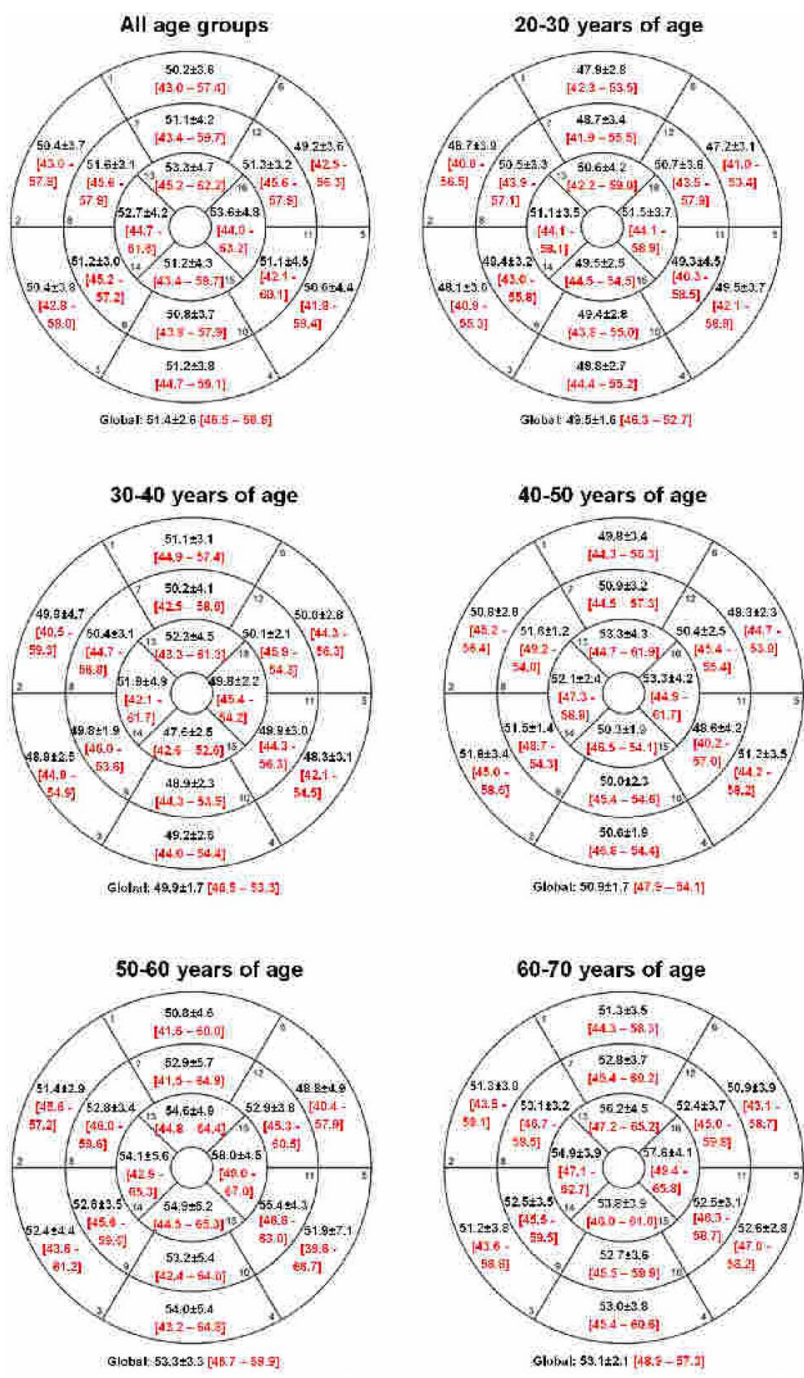


\section{Image 2}
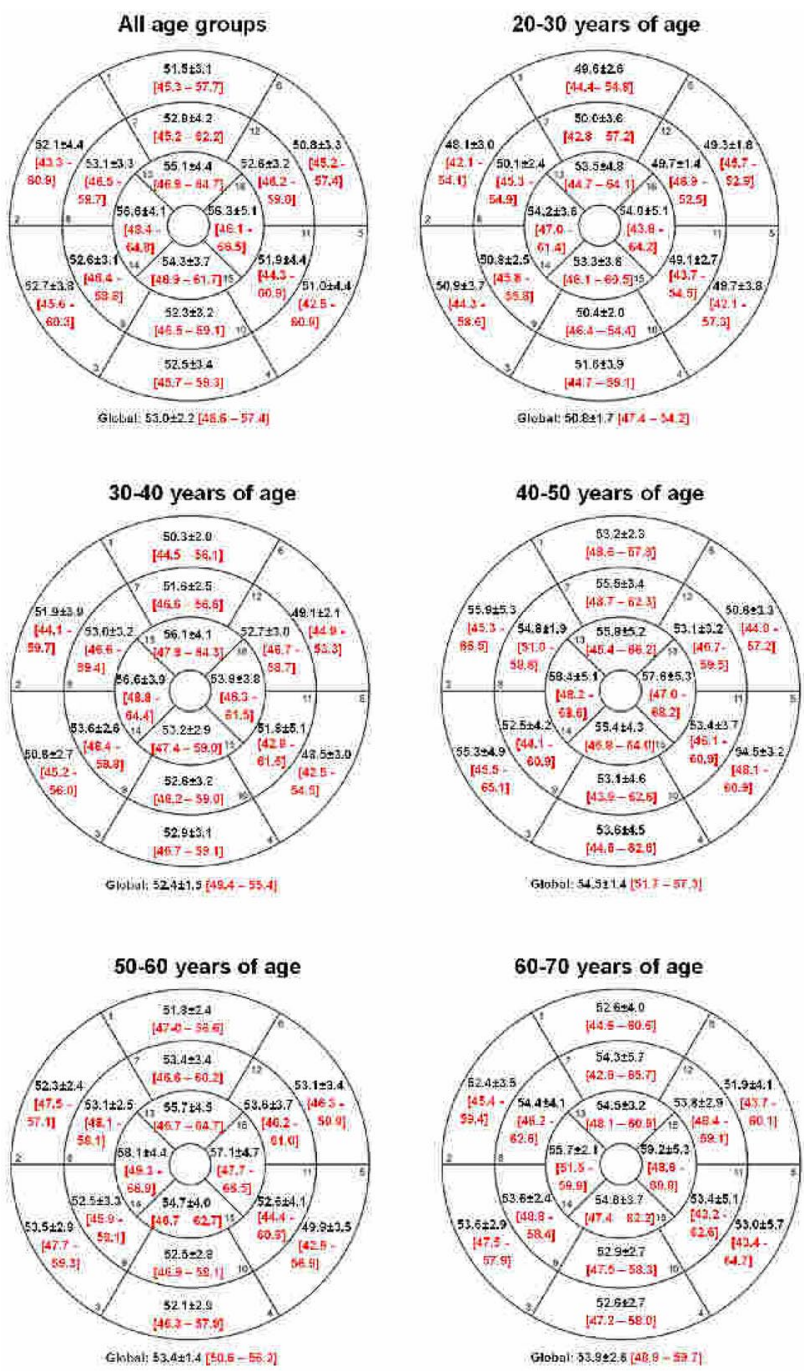

Presenter's first name and last name Alessia Pepe

Presenter's city and country Pisa, Italy

Presenter's email address alessia.pepe@ftgm.it

\section{8}

\section{Changes in CMR parameters and prediction of cardiac complications in thalassemia major: fibrosis tells us more than iron}

\section{Alessia Pepe, Pietro Giuliano, Laura Pistoia, Michela Zerbini, Valentina Vinci, Emanuele Grassedonio, Nicola Giunta, Piera Giovangrossi, Sara Gentili, Alessandra Quota, VINCENZO POSITANO, Antonella Meloni}

Keywords Myocardium, MR/MR-Angiography, Outcomes analysis, Tissue characterisation

\section{Topic Special Focus}

Purpose/Objectives Cardiovascular magnetic Resonance (CMR) has dramatically changed the clinical practice and improved the prognosis in thalassemia major (TM). This is the first study evaluating the predictive value of changes in CMR parameters (myocardial iron, function, and fibrosis) for cardiac complications in TM.

Methods and Materials We followed prospectively 709 TM patients consecutively enrolled in the Myocardial Iron Overload in Thalassemia Network who performed a baseline and a 1st follow up CMR scan after 18 months. Myocardial iron overload (MIO) was measured by multislice multiecho $\mathrm{T} 2 *$ technique and atrial dimensions and biventricular function by cine images. Macroscopic myocardial fibrosis was detected by late gadolinium enhancement technique. Risk classes were defined based on the 4 patterns of MIO from worst to normal.

Results Mean age at baseline CMR was $29.77 \pm 8.53$ years and 374 patients were females. During a mean follow-up of $89.4 \pm 33.3$ months, cardiac events were recorded in 50 (7.1\%) patients: 24 (48\%) episodes of heart failure, 24 (48\%) arrhythmias (23 supraventricular and 1 hypokinetic), and 2 (4.0\%) pulmonary hypertension. Mean time from the 1st follow up CMR to the development of a cardiac complication was $75.31 \pm 35.35$ months. In the univariate Cox regression analysis, cardiac iron cleareance and myocardial fibrosis were identified as univariate prognosticators (Table 1). In the multivariate analysis only myocardial fibrosis remained an independent predictor factor.

Conclusion The presence of myocardial fibrosis at the baseline CMR or developed within 18 months emerges as the strongest long-term predictor for cardiac complications in TM. Our data demonstrate the importance in using the contrast medium for CMR scans in thalassemia patients. Figure 1 shows the classification for the changes detected between baseline and 1st control MRI scans 


\section{Image 1}

Myocardial iron overload

Risk classes based on the 4 patterns of MIO from worst to normal.

For patients with baseline MIO $\rightarrow$ improvement $=$ transition to a better risk class, stabilization $=$ no change in risk class, worsening $=$ transition to a worse risk class.

For patients without baseline $\mathrm{MIO} \rightarrow$ worsening $=$ transition to a worse risk class.

Biventricular ejection fractions

$\rightarrow$ improvement $=\%$ change $>10 \%$, stabilization $=\%$ change between $-10 \%$ and $10 \%$, worsening $=\%$ change $<-10 \%$.

Biventricular volumes, LV mass index, and atrial areas

$\rightarrow$ improvement $=\%$ change $<-10 \%$, stabilization $=\%$ change between $-10 \%$ and $10 \%$, worsening $=\%$ change $>10 \%$

Myocardial fibrosis

$\rightarrow$ considered absent if not detected in any of the two CMRs and present if detected in at least one examination.

Image 2

\begin{tabular}{|c|c|c|}
\hline & \multicolumn{2}{|c|}{ Univariate analysis } \\
\hline & $\operatorname{HR}(95 \% \mathrm{CI})$ & $P$ \\
\hline \multicolumn{3}{|l|}{ Pattern of MO } \\
\hline improvement & Reference & \\
\hline stabilization & $2.07(0.95-4.48)$ & 0.067 \\
\hline worsening & $3.09(1.22-7.86)$ & 0.018 \\
\hline \multicolumn{3}{|l|}{ LVEDVI } \\
\hline improvement & Reference & \\
\hline stabilization & $1.87(0.80-4.33)$ & 0.147 \\
\hline worsening & $2.30(0.91-5.85)$ & 0.080 \\
\hline \multicolumn{3}{|l|}{ LV mass index } \\
\hline improvement & Reference & \\
\hline stabilization & $0.63(0.29-1.35)$ & 0.235 \\
\hline worsening & $1.05(0.51-2.17)$ & 0.890 \\
\hline \multicolumn{3}{|l|}{ LVEF } \\
\hline improvement & Reference & \\
\hline stabilization & $0.99(0.46-2.17)$ & 0.992 \\
\hline worsening & $0.72(0.22-2.38)$ & 0.586 \\
\hline \multicolumn{3}{|l|}{ RVEDVI } \\
\hline improvement & Reference & \\
\hline stabilization & $0.97(0.48-1.98)$ & 0.931 \\
\hline worsening & $1.43(0.65-3.15)$ & 0.373 \\
\hline \multicolumn{3}{|l|}{ RV EF } \\
\hline improvement & Reference & \\
\hline stabilization & $1.64(0.76-3.57)$ & 0.210 \\
\hline worsening & $0.64(0.17-2.40)$ & 0.506 \\
\hline \multicolumn{3}{|c|}{ Left atrial area index } \\
\hline improvement & Reference & \\
\hline stabilization & $1.22(0.53-2.79)$ & 0.640 \\
\hline worsening & $1.13(0.44-2.95)$ & 0.800 \\
\hline \multicolumn{3}{|c|}{ Right atrial area index } \\
\hline improvement & Reference & \\
\hline stabilization & $1.36(0.57-3.29)$ & 0.490 \\
\hline worsening & $1.69(0.61-4.69)$ & 0.312 \\
\hline \multicolumn{3}{|c|}{ Myocardial fibrosis } \\
\hline present & Reference & \\
\hline absent & $1.89(1.09-3.49)$ & 0.041 \\
\hline
\end{tabular}

Presenter's first name and last name

Alessia Pepe

Presenter's city and country

Pisa, Italy

Presenter's email address

alessia.pepe@ ftgm.it

\section{9}

\section{Value of Cardiac Magnetic Resonance Imaging to Differentiate Biopsy-Proven Myocarditis and Dilated Cardiomyopathy}

\section{Jan Michael Brendel, Karin Klingel, Jens Kübler, Sebastian Gassenmaier, Konstantin Nikolaou, Meinrad Gawaz, Simon Greulich, Susann-Cathrin Olthof, Florian Hagen, Patrick Krumm}

Keywords Myocarditis, Dilated Kardiomyopathy, Magnetic Resonance Imaging, Endomyocardial Biopsy, Function, Late Gadolinium Enhancement

Topic Area of Interest

Purpose/Objectives To identify cardiac magnetic resonance (CMR) characteristics to discriminate biopsy-proven: (1) myocarditis from dilated cardiomyopathy (DCM), and (2) different stages of myocarditis (acute vs. chronic vs. healed myocarditis).

Methods and Materials 208 patients (age $51 \pm 15$ years; $24 \%$ female) with endomyocardial biopsies (EMB) and CMR in the years 2010-2020 were evaluated and divided in groups of acute, chronic, healed lymphocytic myocarditis vs DCM. CMR protocol comprised functional and late gadolinium enhancement imaging (LGE).

Results Right ventricular EMB revealed: 1) acute myocarditis $(\mathrm{n}=7,3 \%), 2)$ chronic myocarditis $(\mathrm{n}=117,57 \%)$, $3)$ healed myocarditis $(n=36,17 \%)$, and 4) DCM $(n=48$, 23\%). Left-ventricular ejection fraction (LV-EF) was higher, and LV end-diastolic volume index (LV-EDVI) was lower in myocarditis patients (acute: $41 \%, 83 \mathrm{ml} / \mathrm{m}^{2}$; chronic: $41 \%$, $101 \mathrm{ml} / \mathrm{m}^{2}$; healed: $44 \%, 95 \mathrm{ml} / \mathrm{m}^{2}$ ) compared to DCM patients $\left(29 \%, 131 \mathrm{ml} / \mathrm{m}^{2}\right), \mathrm{p}<0.0001$. Myocarditis patients demonstrated a higher LGE prevalence $(71 \%$ acute; $66 \%$ chronic; $72 \%$ healed) than DCM patients $(40 \%), p=0.006$. Myocarditis patients defined by their histological stage (acute/chronic/healed) showed no significant differences in cine or LGE-CMR characteristics. 
Conclusion CMR can differentiate biopsy-proven lymphocytic myocarditis from DCM by the presence of lower left-ventricular volumes and both higher LV-EF and LGE prevalence. However, cine and LGE CMR alone seem not to be sufficient to discriminate non-invasively different stages of myocarditis (acute/chronic/healed).

\section{References}

Ferreira VM, Schulz-Menger J, Holmvang G, et al. Cardiovascular Magnetic Resonance in Nonischemic Myocardial Inflammation: Expert Recommendations. J Am Coll Cardiol 2018;72:3158-3176.

Grün S, Schumm J, Greulich S, et al. Long-term followup of biopsy-proven viral myocarditis: Predictors of mortality and incomplete recovery. J Am Coll Cardiol 2012;59:1604-1615.

Greulich S, Ferreira VM, Dall'Armellina E, et al. Myocardial Inflammation-Are We There Yet? Curr Cardiovasc Imaging Rep 2015;8:6.

\section{Image 1}

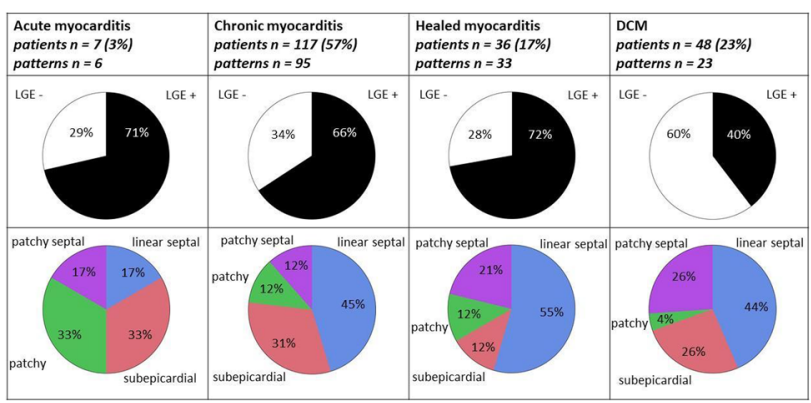

\section{Image 2}

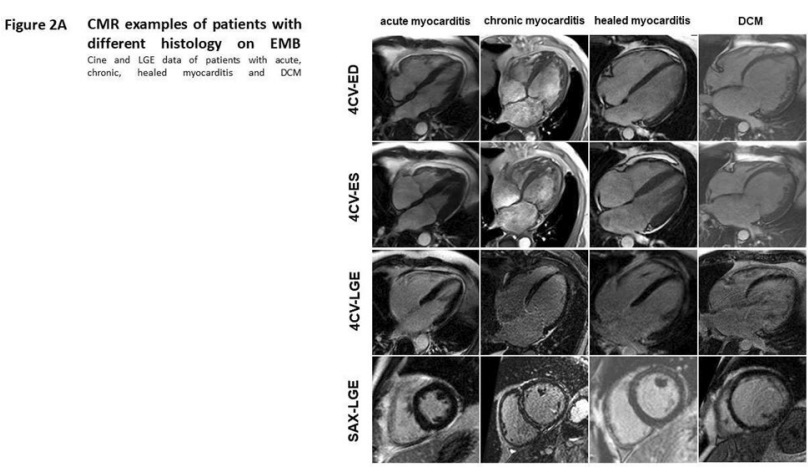

\section{Image 3}

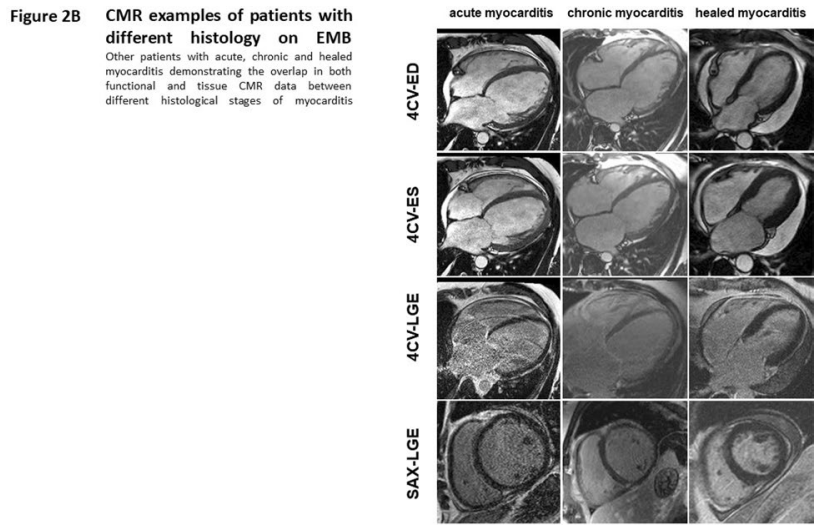

Presenter's first name and last name

Jan Michael Brendel

Presenter's city and country

Tübingen/Germany

Presenter's email address

jan.brendel@brendelhost.de

\section{0}

\section{Deep learning-based image reconstruction for CT angiography of the aorta}

\section{Andra Heinrich, Felix Streckenbach, Ebba Beller, Marc-André Weber, Felix G. Meinel}

Keywords CT/CT-Angiography, Aorta, Deep Learning

Topic Imaging Technique

Purpose/Objectives To evaluate the impact of a novel deep learning-based image reconstruction algorithm on the image quality in CT angiography of the aorta.

Methods and Materials We retrospectively analyzed 51 consecutive patients (median age 69 years, 19 women) who underwent ECG-gated chest CT angiography and non-gated acquisition for the abdomen on a 256-dectector-row CT. Images were reconstructed with adaptive statistical iterative reconstruction (ASIR-V) and deep learning-based image reconstruction (DLIR). Intravascular image noise, signal-to-noise ratio (SNR) and contrast-to-noise ratio (CNR) were quantified 
for the ascending and descending thoracic aorta, the abdominal aorta and the iliac arteries. Two readers scored subjective image quality on a 5 -point scale $(5=$ excellent, $4=$ good, $3=$ sufficient, $2=$ poor, $1=$ non-diagnostic).

Results Compared to ASIR-V, DLIR reduced median image noise by $51-54 \%$ for the ascending ( 22 vs. 48 Hounsfield units (HU)) and the descending thoracic aorta ( 23 vs. $47 \mathrm{HU}$, both $\mathrm{p}<0.0001)$. Correspondingly, median CNR roughly doubled for the ascending aorta $(15 \mathrm{vs}$. $7 \mathrm{HU})$ and descending thoracic aorta (14 vs. $7 \mathrm{HU}$, both $\mathrm{p}<0.0001)$. There was a $38 \%$ reduction in image noise for the abdominal aorta (18 vs. 29HU) and the iliac arteries (13 vs. $21 \mathrm{HU}$, both $\mathrm{p}<0.0001$ ), with a corresponding improvement in CNR (18 vs. 11 and 23 vs. $14 \mathrm{HU}$ respectively, both $\mathrm{p}<0.0001)$. Median subjective image quality improved from good to excellent at all anatomical levels (all $\mathrm{p}<0.0001$ ).

Conclusion In CT angiography of the aorta, DLIR substantially improved objective and subjective image quality beyond what can be achieved by state-of-the-art iterative reconstruction. This can pave the way for further radiation or contrast dose reductions.

\section{Image 1}
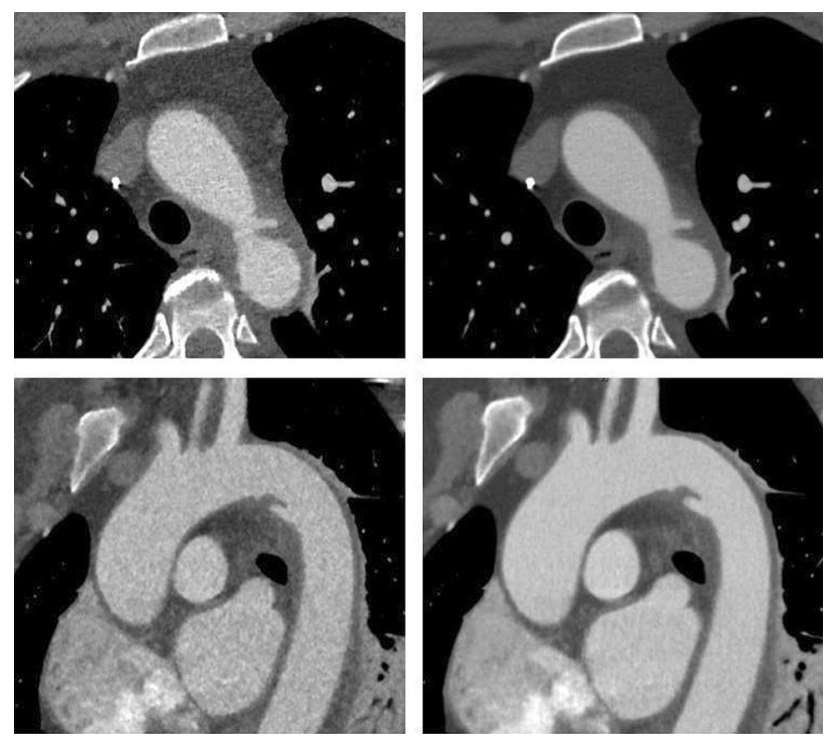

Image 2
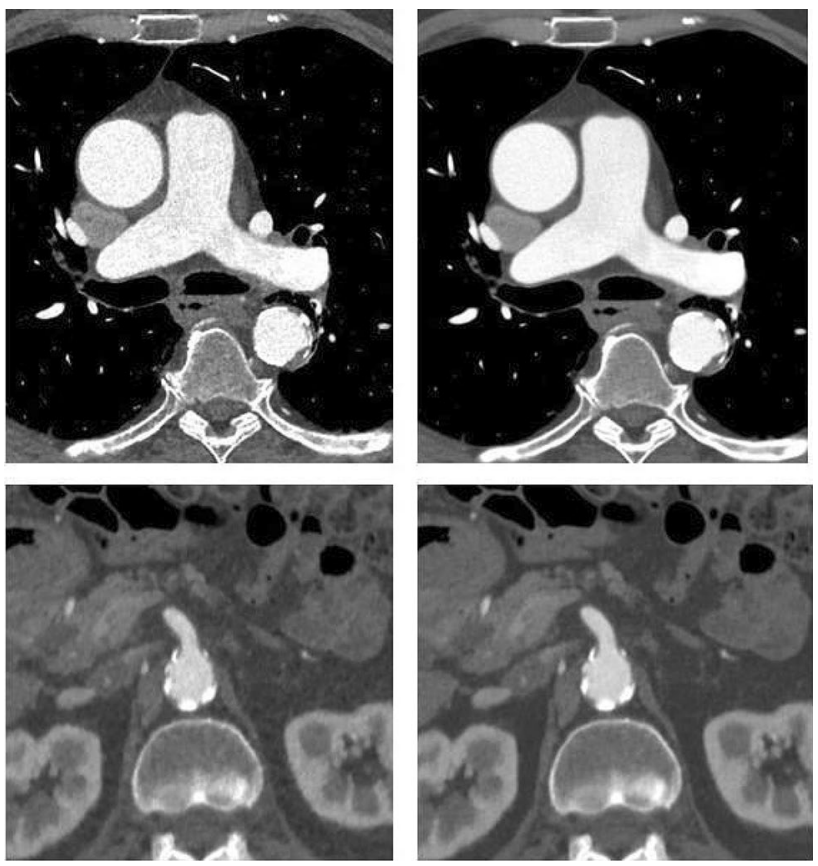

Presenter's first name and last name

Andra Heinrich

Presenter's city and country

Rostock, Germany

Presenter's email address

andra.heinrich@med.uni-rostock.de

\section{1}

Global and regional test-retest reproducibility of native $\mathrm{T} 1$ and $\mathrm{T} 2$ mapping in cardiac magnetic resonance imaging

\section{Benjamin Böttcher, Roberto Lorbeer, Sophia Stöcklein, Ebba Beller, Cajetan I. Lang, Marc-André Weber, Felix G. Meinel}

Keywords Cardiac Magnetic Resonance Imaging, Reproducibility, Mapping, T1, T2 
Topic Imaging Technique

Purpose/Objectives MRI is an invaluable tool for diagnosis and risk stratification in many cardiac diseases [1]. Conventional MR imaging techniques are increasingly supplemented by mapping techniques which permit quantitative assessment of myocardial tissue with measurement of absolute $\mathrm{T} 1, \mathrm{~T} 2$ and $\mathrm{T} 2 *$ relaxation times [2]. The clinical use of mapping techniques requires sufficient reproducibility of measurement results. Mapping derived values will only be useful clinically if the reproducibility is higher (i.e., the measurement error smaller) than the differences between healthy subject and patients with a specific pathology (or between different pathologies that should be differentiated). There is limited data on the test-retest reproducibility of $\mathrm{T} 1$ and $\mathrm{T} 2$ mapping results. Therefore, the purpose of our study was to investigate the global and regional reproducibility of native $\mathrm{T} 1$ and $\mathrm{T} 2$ mapping results and to analyseanalyze the influence of demographic factors, physiological parameters, slice position and myocardial regions.

Methods and Materials In this prospective cohort-study we included 50 healthy volunteers (29 female, 21 male) with a mean age of $39.4 \pm 13.7$ years. Each volunteer underwent two cardiac MRI investigations at $1.5 \mathrm{~T}$ on the same day including T1 mapping using the modified look-locker inversion-recovery (MOLLI) scheme and steady state free precession (SSFP) based T2 mapping. Global T1 and T2 values were quantified for the entire left ventricle. Regression models were used to analyse the influence of demographic and physiological parameters on the reproducibility of global T1 or T2 measurements. Regional T1 and T2 values were measured for each myocardial segment. Additionally, we grouped myocardial segments by slice position (basal, midventricular, apical) and anatomical region (septum, anterior, lateral, inferior wall). Test-retest reproducibility was assessed using intra-class correlation coefficient (ICC) and Bland-Altman statistics. Results Reproducibility was good for global $\mathrm{T} 1$ values (ICC 0.88) and excellent for global T2 values (ICC 0.91). Regression models showed no significant influence of demographic and physiological parameters on reproducibility. Reproducibility of T1 values was excellent (ICC 0.91 ) for the midventricular slice and good for the apical (ICC 0.86) and basal slice (ICC 0.81). Basal slice T1 reproducibility was significantly lower than that of the midventricular slice. Reproducibility of T1 mapping values was highest in the septum (ICC 0.90) compared to the anterior $(0.81)$, lateral (0.86) and inferior (0.86) wall. Anterior wall $\mathrm{T} 1$ reproducibility was significantly lower than that in the septum. For T2 mapping, reproducibility was good for all slice positions (ICC 0.86 for midventricular, 0.83 for basal and 0.80 for apical slice).
Rreproducibility of T2 mapping was significantly lower for the inferior wall (ICC 0.58) than for septum (0.89), anterior (0.85) and lateral (0.87) wall.

Conclusion Native T1 and T2 mapping showed good to excellent reproducibility with significant regional differences. Based on our data, we recommend global quantitative analysis with suitable commercially available software tools. If these are not available, we recommend averaging regions of interest in the left ventricular septum over three short-axis slices.

\section{References}

Doherty JU, Kort S, Mehran R, et al. ACC/AATS/AHA/ ASE/ASNC/HRS/SCAI/SCCT/SCMR/STS 2019 appropriate use criteria for multimodality imaging in the assessment of cardiac structure and function in nonvalvular heart disease: A report of the ACC Appropriate Use Criteria Task Force, AATS, AHA, ASE, ASNC, HRS, SCAI, SCCT, SCMR and the STS. The Journal of thoracic and cardiovascular surgery 2019:157:e153-e182.

Messroghli DR, Moon JC, Ferreira VM, et al. Clinical recommendations for cardiovascular magnetic resonance mapping of T1, T2, T2* and extracellular volume: A consensus statement by the Society for Cardiovascular Magnetic Resonance (SCMR) endorsed by the European Association for Cardiovascular Imaging (EACVI). Journal of cardiovascular magnetic resonance: official journal of the Society for Cardiovascular Magnetic Resonance 2017:19:75.

\section{Image 1}

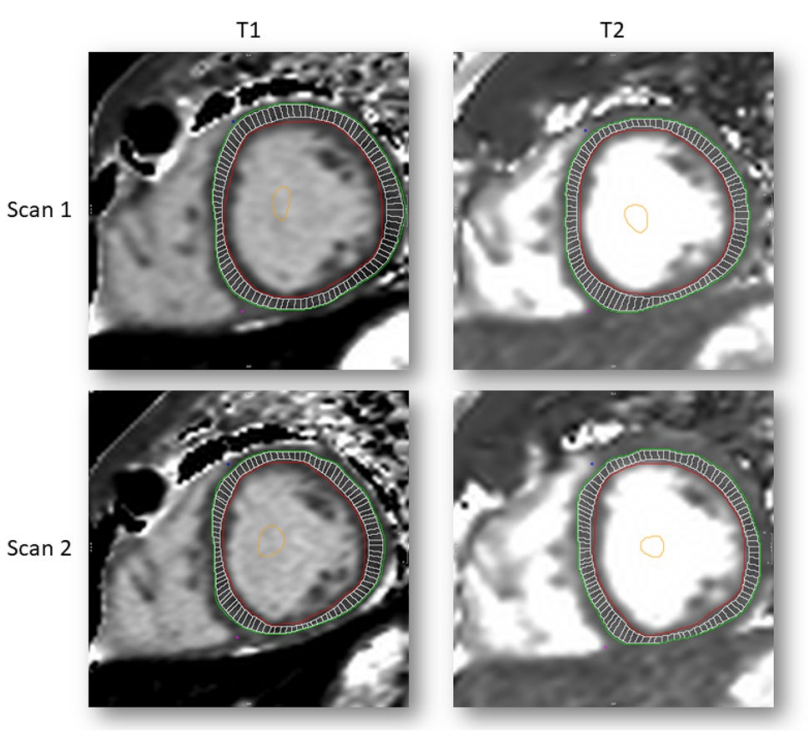


Image 2

T1

T2

A. Reproducibility by myocardial segments
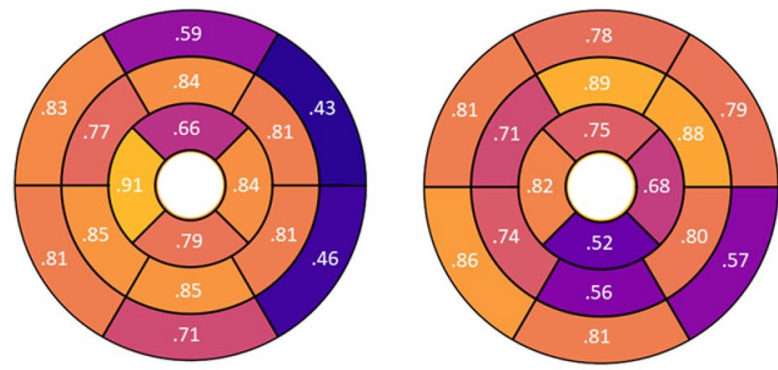

B. Reproducibility by slice position
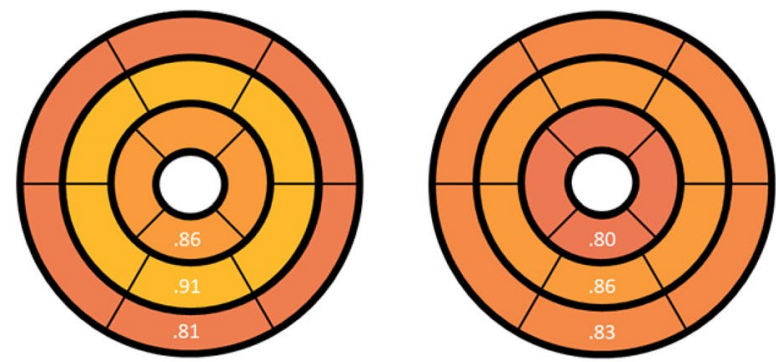

C. Reproducibility by anatomical region

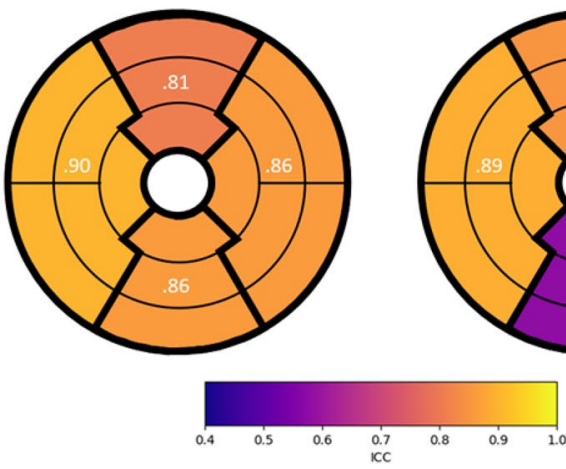

Presenter's first name and last name Benjamin Böttcher

Presenter's city and country

Rostock, Germany

Presenter's email address

benjamin.boettcher@uni-rostock.de
0012

\section{Comparison of two sequences for myocardial T1 mapping and calculation of extracellular volume with MRI}

Alexander Brendel, Jens Kübler, Sebastian Gassenmaier, Florian Hagen, Jan Michael Brendel, Konstantin Nikolaou, Susann-Cathrin Olthof, Petros Martirosian, Simon Greulich, Patrick Krumm

Keywords MR, T1 Mapping, MOLLI, CMR, Extracellular Volume

Topic Imaging Technique

Purpose/Objectives To systematically compare two modified Look-Locker inversion recovery (MOLLI) T1 mapping sequences and their impact on myocardial $\mathrm{T} 1$ values native, post contrast agent application (pCA) and on calculation of extracellular volume (ECV).

Methods and Materials 163 patients (age $55 \pm 17$ years; $38 \%$ female) underwent CMR at $1.5 \mathrm{~T}$ for ischemic, nonischemic cardiomyopathy and myocarditis. Two T1 mapping MOLLI acquisition schemes 5(3)3; and 4(1)3(1)2 were sequentially acquired in mid-ventricular short-axis slice native and 15-20 min pCA. ECV was calculated offline with blood sample hematocrit. Both acquisition schemes were compared in vitro with an inversion recovery fast spin echo (IR-FSE) sequence with a sample series of $\mathrm{MnCl} 2$ phantom. Results Myocardial T1 native for 5(3)3 was $1017 \pm 42 \mathrm{~ms}$; vs. $956 \pm 40 \mathrm{~ms}$ for 4(1)3(1)2; mean intraindividual difference $-61 \mathrm{~ms} ; \mathrm{p}<0.0001$. Myocardial T1 pCA for 5(3)3 was $494 \pm 48 \mathrm{~ms}$, vs. $490 \pm 45 \mathrm{~ms}$ for 4(1)3(1)2; mean difference $-4 \mathrm{~ms} ; \mathrm{p}=0.0006$. Intraindividual decrease from native to pCA T1 for $5(3) 3$ was $523 \pm 62 \mathrm{~ms}$, vs. $466 \pm 59 \mathrm{~ms}$ with 4(1)3(1)2; mean difference $-57 \mathrm{~ms} ; \mathrm{p}<0.0001$. Myocardial ECV for 5(3)3 was $27.6 \pm 4 \%$, vs. $27 \pm 4 \%$ for $4(1) 3(1) 2$; mean difference -0.6 percentage points; $p<0.0001$. Ex vivo T1-values for $\mathrm{MnCl} 20.05 \mathrm{mM}: 1467 \mathrm{~ms}$ IR-FSE; $1439 \mathrm{~ms}$ 5(3)3; $1354 \mathrm{~ms} 4(1) 3(1) 2$. T1-values for $\mathrm{MnCl} 20.3 \mathrm{mM}$ : $431 \mathrm{~ms}$ IR-FSE; $406 \mathrm{~ms}$ 5(3)3; $408 \mathrm{~ms}$ 4(1)3(1)2.

Conclusion T1 MOLLI 5(3)3 acquisition scheme provides reliable estimation of $\mathrm{T} 1$ for long and short $\mathrm{T} 1$ values in vitro as well as in vivo. T1 MOLLI 4(1)3(1)2 scheme underestimates systematically and relevantly higher native myocardial $\mathrm{T} 1$ values in vitro and in vivo. 
This underestimation has only a minor but statistically significant impact on ECV calculation.

\section{Image 1}

Clinical cardiac T1 \& ECV maps
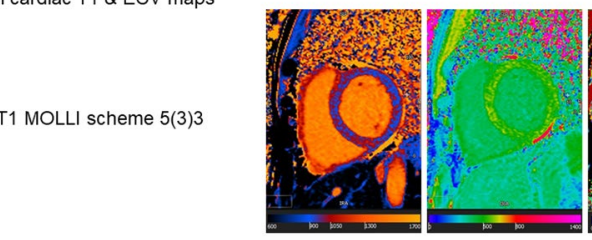

post contrast

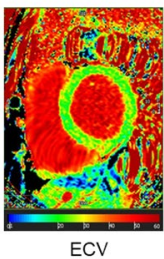

native
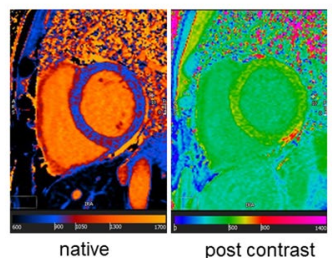

post contrast

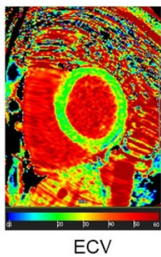

Image 2

Phantom study T1 maps
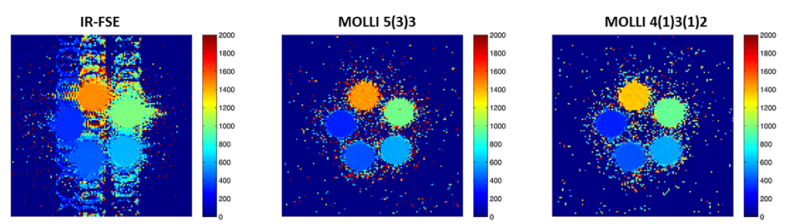

Presenter's first name and last name

Alexander Brendel

Presenter's city and country

Tübingen, Germany

Presenter's email address

alexander.brendel@student.uni-tuebingen.de

\section{5}

\section{Psoas Cross-sectional Measurements Using Manual CT Segmentation Before and After Endovascular Aortic Repair (EVAR)}

\section{Francesco Secchi, Caterina Beatrice Monti, Gabriele Maria Nicolino, Luca Sconfienza, Francesco Sardanelli, Giovanni Nano, Giuseppe Banfi, Mattia Glauber, Massimiliano Marrocco-Trischitta}

Keywords Aorta, CT/CT-Angiography, Aneurysm

Topic Area of Interest

Purpose/Objectives To quantify sarcopenia with psoas muscles manual cross sectional segmentation in CT before and after EVAR and to evaluate the inter and intraobserver agreement between two readers.

Methods and Materials Among 50 patients who underwent CT before and after EVAR, total psoas area (TPA) and psoas density (PMD) were manually measured at level of inferior endplate of 4th lumbar vertebra (L4). Only in pre-procedure $\mathrm{CT}$ intra and inter-reader reliability of psoas segmentation was determined through independent outline of the same abdominal CT images by two radiologist residents (R1, R2) who were blinded to each other's measurement. One reader repeated psoas measurements after two weeks (TPA-PMD 1 and 2). Data are expressed as median \pm interquartile range. Wilcoxon test was used to compare measurements before and after ECVAR. Reproducibility was evaluated using Bland-Altman method.

Results 42 male (84\%) and 8 female patients (16\%) with average age of 42 (range 66-78 years) were studied. Comorbidities were: hypertension (80\%), diabetes (14\%), CAD (28\%), COPD (30\%), kidney failure (12\%). R1 measured at L4: TPA $1=2535.5 \mathrm{~mm} 2(2067.2-2818 \mathrm{~mm} 2)$ and PMD1 $=34.5$ HU $(26.6-37.8 \mathrm{HU}) ;$ TPA2 $=2631.5 \mathrm{~mm} 2$ (2050.2-2877.7 $\mathrm{mm} 2$ ) and PMD2 $=32 \mathrm{HU}$ (25.1-37.5 HU). Inter-observer reproducibility of TPA showed a bias of 64.0 and Cor of 359.2; reproducibility of PMD showed a bias of -2.43 and Cor of 6.19. Intra-observer reproducibility of TPA showed a bias of -81.1 and Cor of 394.6; reproducibility of PMD showed a bias of 1.41 and Cor of 
6.36. TPA before and after EVAR changes from $1006 \mathrm{~mm} 2$ (1243-1445 mm2) to $937 \mathrm{~mm} 2(1102-1331 \mathrm{~mm} 2)$ with a significant difference $(\mathrm{p}<0.001)$. PMD before and after EVAR changes from $26.5 \mathrm{HU}(33-38.7 \mathrm{HU})$ to $26 \mathrm{HU}$ (32-37 HU) without a significant difference $(\mathrm{p}=0.630)$.

Conclusion A significant reduction of TPA after EVAR was found. A good intra and inter reader reproducibility were observed in the measurement of both TPA and PMD.

Presenter's first name and last name

Francesco Secchi

Presenter's city and country

Milan, Italy

Presenter's email address

francesco.secchi@unimi.it

\section{6}

\section{Histological validation of dark-blood late gadolinium enhancement MRI without additional magnetization preparation}

\section{Robert J. Holtackers, Suzanne Gommers, Luuk I.B. Heckman, Caroline M. Van De Heyning, Amedeo Chiribiri, Frits W. Prinzen}

Keywords magnetic resonance imaging, late gadolinium enhancement, myocardial infarction

\section{Topic Imaging Technique}

Purpose/Objectives For already two decades, late gadolinium enhancement (LGE) MRI has been considered the reference standard for the non-invasive assessment of myocardial viability. A major drawback of conventional bright-blood LGE, however, is the poor contrast between scar tissue and the blood pool. Since both tissues may have similar highintensity signal, determining the scar-blood barrier is often challenging. In cases with subendocardial scar or papillary muscle scar, the apparent scar volume can be substantially underestimated or even remain completely undetected [1]. Recently, a dark-blood LGE method was developed which increases scar-to-blood contrast without using additional magnetisation preparation, making it widely and readily available for clinical routine use [2]. In a cohort of 300 patients, this novel LGE method demonstrated superior sensitivity for ischaemic scar tissue compared to conventional LGE [3]. In this study, we aim to histologically validate this novel dark-blood LGE method in a porcine animal model with induced myocardial infarction.
Methods and Materials In 13 Yorkshire pigs, myocardial infarction was experimentally induced by transient coronary artery occlusion. At 7-weeks post-infarction, the animals underwent MRI examination under general anesthesia using a 1.5 T MR system (Ingenia, Philips Healthcare, NL). Approximately ten minutes after intravenous injection of $0.2 \mathrm{mmol} / \mathrm{kg}$ gadobutrol (Gadovist, Bayer Pharmaceuticals, DE), conventional bright-blood LGE and dark-blood LGE images were acquired. A 1:1 randomisation scheme was used to decide the order of acquisition of the two LGE methods. Following MRI examination, the animals were sacrificed and histopathology was obtained. Matching LGE slices and histopathology samples were selected based on anatomical landmarks. Independent observers, while blinded to other data, manually delineated the endocardial, epicardial, and infarct borders on either LGE images or histopathology samples. Infarct size as measured by conventional bright-blood LGE and novel dark-blood LGE were compared with histopathology as reference standard on a per-slice basis. Contrast-to-noise ratios were calculated for both LGE methods. Results A combined total of 24 matched LGE and histopathology slices were available for histopathological validation. Examples of in-vivo LGE MRI and corresponding histopathology samples are shown in Fig. 1. Infarct size by dark-blood LGE and conventional bright-blood LGE correlated strongly with infarct size by histopathology $(r=0.998$ and $\mathrm{r}=0.892$, respectively, Fig. 2A). Although Bland-Altman analyses demonstrated high levels of agreement for both comparisons with histopathology (Fig. 2B), conventional LGE showed a significant bias of $-1.57 \%(\mathrm{p}=0.03)$. In contrast, dark-blood LGE showed no significant bias when compared against histopathology $(-0.03 \%, \mathrm{p}=0.75)$. The 95\% limits of agreement were smaller for dark-blood LGE (-0.90\%, 0.84\%) compared to conventional brightblood LGE (-8.02\%, 4.88\%). Imaging examples of conventional bright-blood LGE and dark-blood LGE imaging are shown in Fig. 3. CNR analysis demonstrated a significant increase of $106 \%$ in scar-to-blood contrast for dark-blood LGE compared to conventional bright-blood LGE (8.14 vs 3.96, p $<0.001$, Fig. 4).

Conclusion Dark-blood LGE without additional magnetization preparation provides superior visualization and quantification of ischemic scar patterns compared to the current in-vivo reference standard. Infarct size as assessed by darkblood LGE demonstrated superior correlation and agreement compared to conventional bright-blood LGE, using histopathology as reference standard. Since this dark-blood LGE method does not require any additional magnetization preparation, it guarantees an easy and swift implementation in every clinical routine setting as a potential replacement method for conventional bright-blood LGE. 


\section{References}

Kellman P. Dark-blood late-enhancement imaging improves detection of myocardial infarction. JACC Cardiovasc Imaging 2018;11(12):1770-2. https://doi.org/10.1016/j.jcmg. 2017.10.014.

Holtackers RJ, Chiribiri A, Schneider T, Higgins DM, Botnar RM. Dark-blood late gadolinium enhancement without additional magnetization preparation. J Cardiovasc Magn Reson 2017;19(1):64. https://doi.org/10.1186/ s12968-017-0372-4.

Holtackers RJ, Van De Heyning CM, Nazir MS, Rashid I, Ntalas I, Rahman H, Botnar RM, Chiribiri A. Clinical value of dark-blood late gadolinium enhancement cardiovascular magnetic resonance without additional magnetization preparation. J Cardiovasc Magn Reson 2019;21(1):44. https://doi.org/10.1186/s12968-019-0556-1.

\section{Image 1}

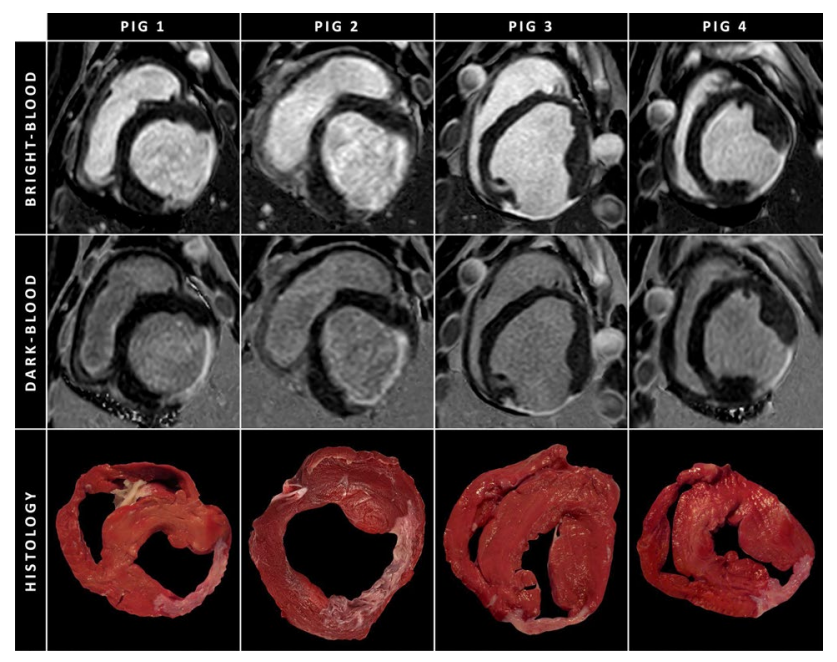

\section{Image 2}
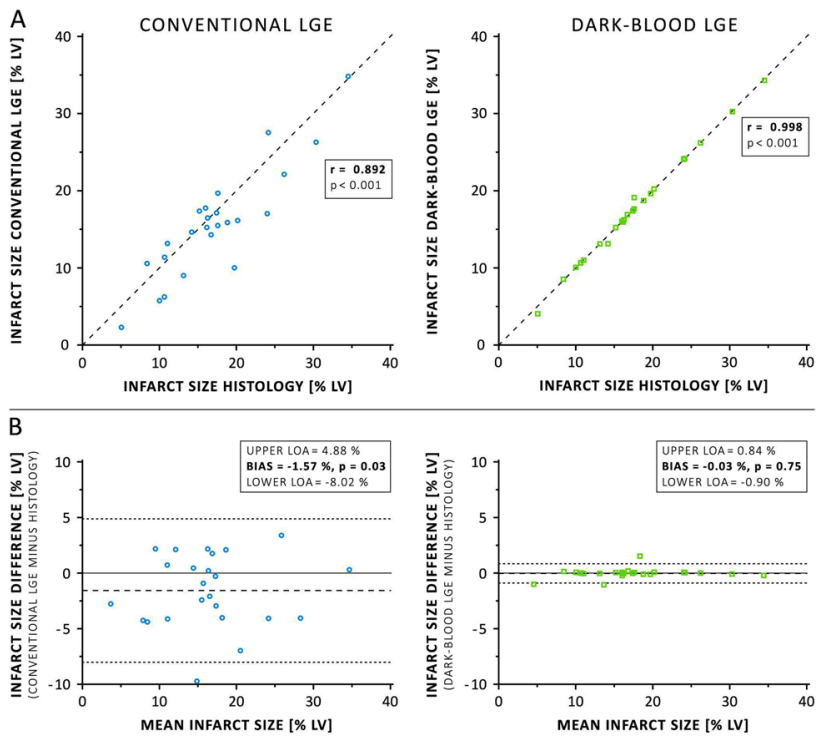

\section{Image 3}

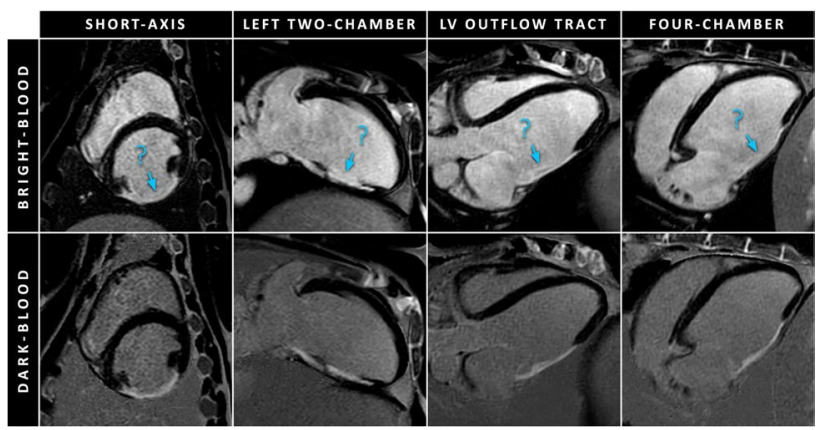




\section{Image 4}

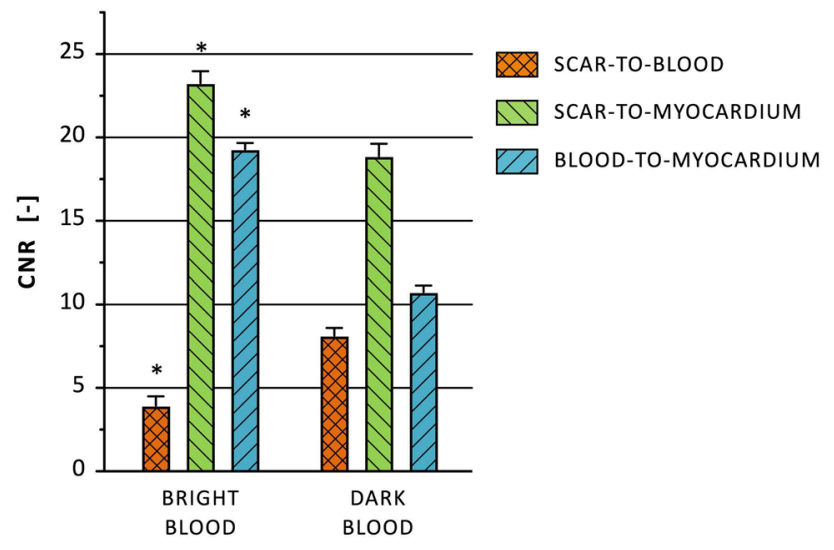

Presenter's first name and last name

Robert J. Holtackers

Presenter's city and country

Maastricht, the Netherlands

Presenter's email address

rob.holtackers@mumc.nl

\section{8}

\section{Differentiating Left Ventricular hypertrophic phenotype. CMR lead the way. A single center experience}

\section{Niki Lama, Vasileios Patris, Efstratios Karavasilis, Mihalis Argiriou, E. Alexopoulou, STAVROS SPILIOPOULPOS, Nikolaos L. Kelekis}

Keywords Myocardium, MR/MR-Angiography, Diagnostic procedure, Outcomes analysis, Cardiomyopathies

\section{Topic Special Focus}

Purpose/Objectives Left ventricular hypertrophy (LVH) is related with various underlying pathological conditions. Common causes of LVH are high blood pressure (hypertension), aorticvalve disease, infiltrative cardiomyopathies (amyloidosis, Fabry disease, sarcoidosis),hypertrohic cardiomyopathy (HCM) and athlete's heart (a condition correlated to physical workout). It is not unusual echocardiographic appearance and clinical characteristics of LV hypertrophy to resemble. CMR has high spatial resolution and ability to deliver a comprehensive knowledge about cardiac anatomic structures, function, and tissue characterization. The present study was undertaken, attempting to highlight the value of CMR contribution on investigation of underlying causes of LVH.

Methods and Materials 121 patients with LVH were retrospectively included to our research, as well as a small group of 22 healthy individuals as controls. All patients underwent a CMR examination using a 3.0 T MRI Philips (Achieva TX) manufactured scanner equipped with a 16-channel XL-Torso 2 anterior and posterior receiver coils. A standard volumetric study was performed followed by a native T1 mapping 4-chamber long-axis image and 3 short-axis slices using a modified look-locker inversion recovery sequence after regional shimming. After a bolus of contrast $(0.1 \mathrm{mmol} / \mathrm{kg}$ gadoterate meglumine, Dotarem, Guerbet S.A., France) and standard late gadolinium enhancement imaging, T1 mapping was repeated $15 \mathrm{~min}$ post-contrast using the same slice locations with the modified look-locker inversion recovery sequence, to produce automated inline ECV mapping reconstruction T1-mapping protocols used $5 \mathrm{~s}(3 \mathrm{~s}) 3 \mathrm{~s}$ and $4 \mathrm{~s}(1 \mathrm{~s}) 3 \mathrm{~s}(1 \mathrm{~s}) 2 \mathrm{~s}$ sampling, pre- and post-contrast, respectively. Image postprocessing was performed using commercially available clinical CMR software CVI 42 (Circle Cardiovascular Imaging, Calgary, Canada).

Results We analyzed all CMR findings of each patient, as heart function, cavities dimensions, thickness of the myocardium, and nevertheless tissue characterization. Although morphological appearance (restrictive or dilated), and hypertrophic myocardial pattern may give some clue, we concluded that the most valuable features were late gadolinium enhancement (LGE) images and novel approaches (myocardial mapping). We were able to detect, in concordance always with patient's clinical history, 49 cases of aortic valve stenosis (AS), 39 cases of Amyloidosis, 14 cases of HCM, 9 cases of Sarcoidosis, 4 cases of Hypertensive cardiomyopathy, 2 cases of athlete's heart, and 1 case of Fabry disease, as well as 3 cases that we could not direct diagnosis.

Conclusion As we expected, we concluded that tissue characterisation was the most important tool in differentiation process. Although LGE images were extremely valuable, when we combined them with mapping techniques (T1 native and ECV), completely upgraded our diagnostic confidence. So, we would like to focus attention on integration of multiparametric mapping on diagnostic workflow, especially when cases with LVH are studied. 


\section{Image 1}

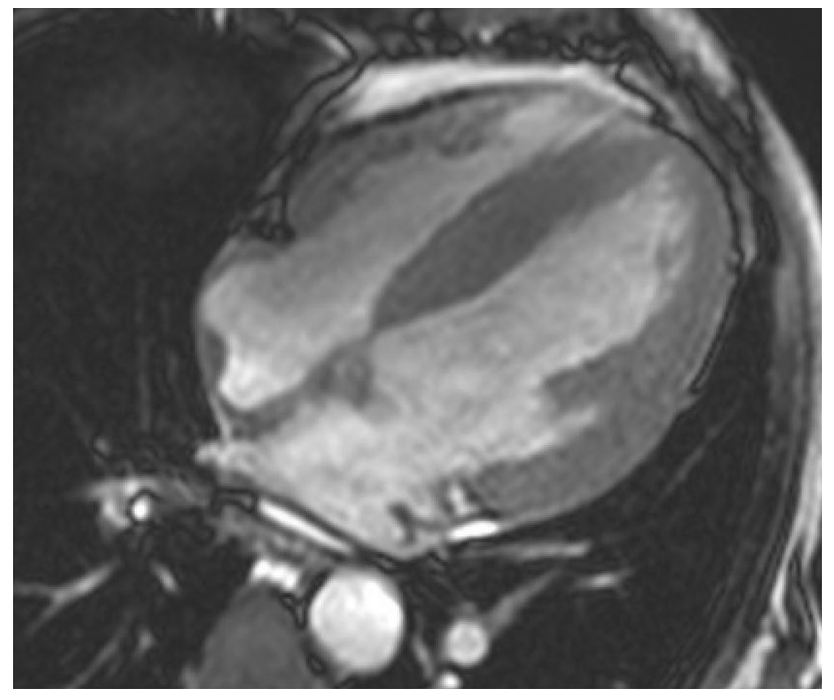

\section{Image 2}

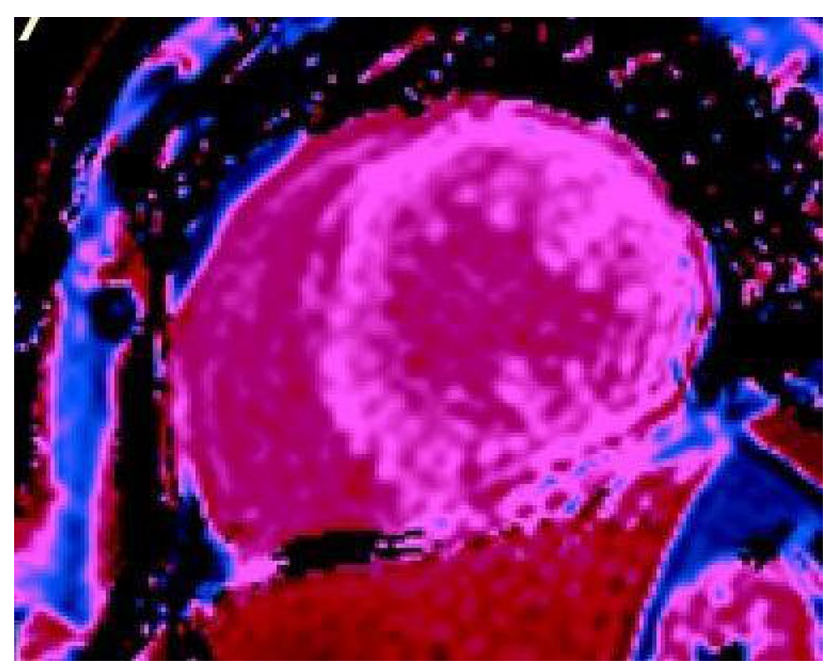

\section{Image 3}

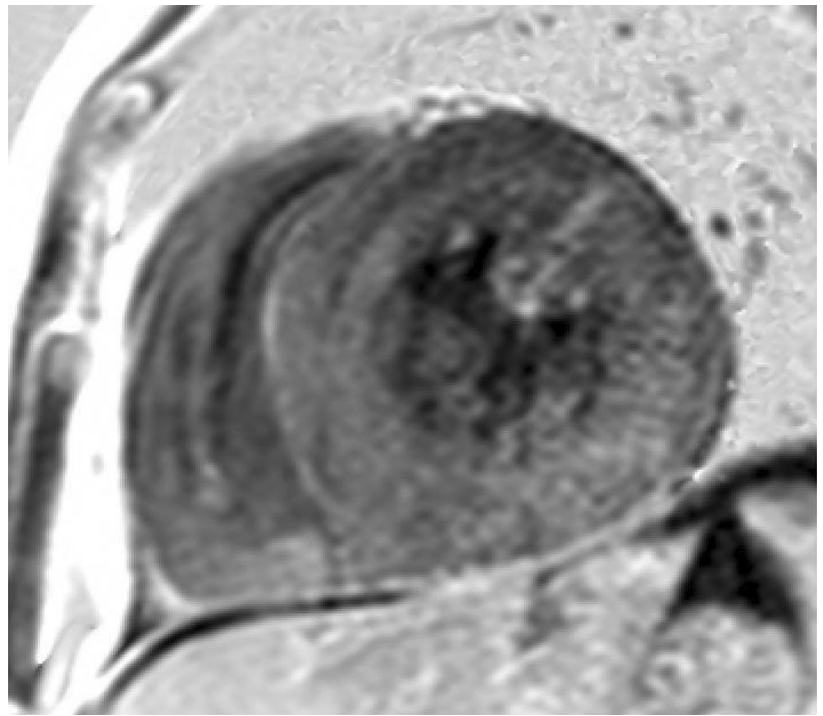

\section{Image 4}

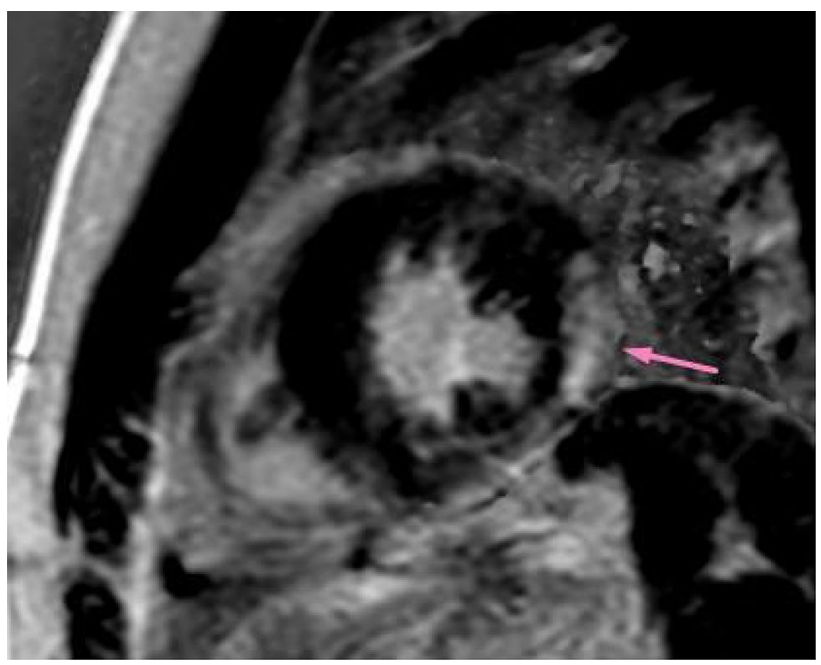


Presenter's first name and last name

Niki Lama

Presenter's city and country

Athens, Greece

Presenter's email address

niklampatr@gmail.com

\section{9}

\section{Splenic Sarcoidosis revealed during CMR. The unexpected!}

\section{Niki Lama, Elias Gialafos, Efstratios Karavasilis, Varvara Pantoleon, Maria Mademli, Nikolaos L. Kelekis}

Keywords MR/MR-Angiography, Diagnostic procedure, Education, Outcomes analysis

\section{Topic Special Focus}

Purpose/Objectives Sarcoidosis is a multi-systematic inflammatory disease, affecting not only the lungs. During CMR studies splenic lesions can be detected incidentally. The aim of our study is to highlight the features of splenic sarcoidosis, revealed to us during Cardiac Magnetic Resonance studies (CMR), providing a differentiation assistance for feature use.

Methods and Materials We reviewed 120 patients scanned in our MRI Unit during the last two year, as referrals for CMR scans, in the presence of known lung sarcoidosis to determine the possibility of cardiac involvement. We focus our research only in those with splenic lesions, and we were able to detect 6 patients with distinct splenic features. All patients underwent a CMR examination using a $3 \mathrm{~T}$ MRI scanner Achieva (Philips Medical Systems, Best, The Netherlands). Protocol included cine imaging; T2 mapping (T2-GraSE); T1 maps, native and late gadolinium enhanced (15 min post Gd), using modified Look-Locker Inversion recovery (MOLLI) sequence; and an ECG triggered phase sensitive inversion recovery (PSIR) (IR value selected after Look-Locker acquisition) $10 \mathrm{~min}$ after the gadolinium injection. Image analysis was performed using commercially available clinical CMR software CVI 42 (Circle Cardiovascular Imaging, Calgary, Canada).

Results Described splenic involvement in sarcoidosis, usually presents as either splenomegaly or rarer as multiple focal splenic lesions. Patients can be asymptomatic, or they can present with systemic symptoms. The distinctive pattern that we acknowledge, was the presence of multiple nodular lesions with low signal intensity in T2W and STIR images. Hyperenhancement was noticed in post contrast delayed enhancement images (PSIR), either with ring-like, or with complete enhancement of the nodule. Time were higher on nodules compering to the rest splenic parenchyma on $\mathrm{T} 1$ native map. ECV map highlighted normal values on splenic parenchyma, with increased values only on nodules. Their size ranged from few $\mathrm{mm}$ to $2 \mathrm{~cm}$. There was no correlation between dimensions of spleen and the presence of nodules. In one case we were also able to track down, the scan after one year of treatment, which highlighted the decreased size of nodules as well as their reduced number.

Conclusion We would like to present the poorly described, until now, characteristics on splenic sarcoidosis. This can be used as a differentiation tool, in future studies acquired during assessment of multiple focal lesions in splenic parenchyma.

Presenter's first name and last name Niki Lama

Presenter's city and country Athens, Greece

Presenter's email address niklamapatr@gmail.com

\section{1}

\section{Acute Aortic Root Injury from Coronary Interventions - Early and Follow-up CT Imaging Findings}

\section{Luca Cappellini, Matthias Eberhard, Christian Templin, Paul Robert Vogt, Robert Manka, Hatem Alkadhi}

Keywords Intramural hematoma, Computed tomography, Catheterization procedure, Coronary artery, Aorta

Topic Imaging Technique

Purpose/Objectives Aortic injury represents a rare but potentially fatal complication of invasive coronary angiography. This monocentric, retrospective, observational study evaluated early and follow-up CT imaging findings of patients with aortic root injury caused by interventional coronary procedures.

Methods and Materials We included patients who underwent early and follow-up CT after aortic injury from invasive coronary intervention between January 2019 and January 2021. All patients received non-contrast and contrast-enhanced electrocardiography-gated CT within 
$2 \mathrm{~h}$ of the intervention; follow-up CT was performed after 2-8 days.

Results Five patients were included (median age 70 years, 3 female). The most common injury after intervention was intramural hematoma (IMH) $(4 / 5,80 \%)$, followed by classic aortic dissection $(1 / 5,20 \%)$. In all patients with IMH, there was accumulation of hyperdense, undiluted contrast media in the wall of the aortic root from invasive angiography. In the patient with classic aortic dissection layering of undiluted contrast media from invasive angiography in the false lumen was present. Aortic root injury was classified as Dunning type I in $20 \%$, type II in $20 \%$, type III in $60 \%$, and Sueyoshi category III in $20 \%$ of patients. Follow-up CT showed complete resolution of contrast media accumulation in all four patients under conservative management. The patient with aortic dissection underwent emergency replacement of the ascending aorta.

Conclusion CT of iatrogenic aortic root injury from invasive coronary intervention showed in the majority of patients a variant IMH with accumulation of undiluted, hyperdense contrast media in the wall of the aortic root, showing favorable short-term outcome.

\section{References}

Dunning DW, Kahn JK, Hawkins ET, O'Neill WW. Iatrogenic coronary artery dissections extending into and involving the aortic root. Catheter Cardiovasc Interv 2000;51(4):387-393. https://doi.org/10.1002/1522726x(200012)51:4<387::aid-ccd3>3.0.co;2-b

Doll JA, Hira RS, Kearney KE, Kandzari DE, Riley RF, Marso SP, Grantham JA, Thompson CA, McCabe JM, Karmpaliotis D, Kirtane AJ, Lombardi W. Management of Percutaneous Coronary Intervention Complications: Algorithms From the 2018 and 2019 Seattle Percutaneous Coronary Intervention Complications Conference. Circ Cardiovasc Interv 2020;13(6):e008962. https://doi.org/10.1161/ CIRCINTERVENTIONS.120.008962

Murillo H, Molvin L, Chin AS, Fleischmann D. Aortic Dissection and Other Acute Aortic Syndromes: Diagnostic Imaging Findings from Acute to Chronic Longitudinal Progression. Radiographics 2021;41(2):425-446. https://doi. org/10.1148/rg.2021200138

Ueda T, Chin A, Petrovitch I, Fleischmann D. A pictorial review of acute aortic syndrome: discriminating and overlapping features as revealed by ECG-gated multidetectorrow CT angiography. Insights Imaging 2012;3(6):561-571. https://doi.org/10.1007/s13244-012-0195-7

\section{Image 1}

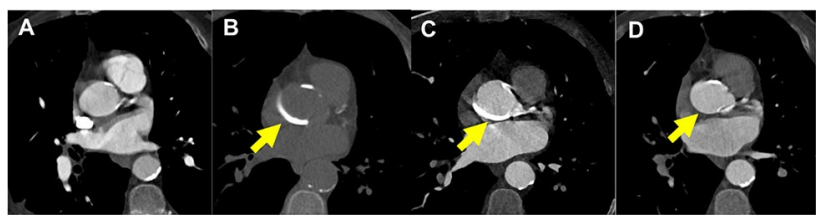

Presenter's first name and last name Luca Cappellini

Presenter's city and country

Milan, Italy

Presenter's email address

lucaalessandro.cappellini@gmail.com

\section{2}

\section{A novel approach to determine aortic valve area with phase contrast cardiovascular magnetic resonance imaging}

\section{Felix Troger, Ivan Lechner, Martin Reindl, Christina Tiller, Magdalena Holzknecht, Mathias Pamminger, Christian Kremser, Sebastian Reinstadler, Axel Bauer, Bernhard Metzler, Agnes Mayr, Gert Klug}

Keywords Cardiac Magnetic Resonance Imaging, PhaseContrast CMR, Aortic Valve Stenosis, Valvular Heart Disease

\section{Topic Area of Interest}

Purpose/Objectives Transthoracic echocardiography (TTE) has become the diagnostic standard for evaluating aortic stenosis (AS) severity, mainly because of its advantages in comparison to the gold standard of cardiac catheterization. However, its inaccuracies in determining stroke volume (SV) and consequentially computing aortic valve area (AVA) call for a more precise and dependable method. Phase contrast cardiovascular magnetic resonance imaging (PC-CMR) is an aspiring tool to push these boundaries. The aim of this study was to validate a novel and simple approach based on PC-CMR against the invasive and echocardiographic determination of SV and AVA in patients with moderate and severe AS.

Methods and Materials A total of 50 patients with moderate or severe AS underwent TTE, cardiac catheterization 
and CMR; AVA by PC-CMR was determined via plotting momentary flow across the valve against momentary flow velocity. SV via CMR was measured directly via PC-CMR and volumetrically using cine images. Invasive SV and AVA were determined via Fick principle and Gorlin formula, respectively. TTE yielded SV and AVA using the continuity equation. Finally, gradients were calculated via the modified Bernoulli equation.

Results SV by PC-CMR showed a strong correlation with cine-CMR with no significant bias (r: 0.730, $<<0.001$; SV by PC-CMR: $85 \pm 31 \mathrm{ml}$; SV by cine-CMR: $85 \pm 19 \mathrm{ml}$, $\mathrm{p}=0.829)$. Peak gradients determined by PC-CMR were $65 \pm 29 \mathrm{mmHg}$ and correlated inversely with AVA by PC-CMR ( $\mathrm{r}:-0.371 ; \mathrm{p}=0.008$ ). Mean AVA during the whole systolic phase showed a moderate correlation (r: $0.544, p<0.001$ ) to invasive AVA with a small bias (AVA by CMR: $0.78 \pm 0.25 \mathrm{~cm}^{2}$ versus invasive AVA: $0.70 \pm 0.23 \mathrm{~cm}^{2}$, bias: $0.08 \mathrm{~cm}^{2}, p=0.017$ ). Inter-methodical correlation and bias of AVA as measured by TTE and invasive AVA (AVA by TTE: $0.81 \pm 0.23 \mathrm{~cm}^{2}, \mathrm{r}: 0.580$, $\mathrm{p}<0.001$, bias $0.11 \mathrm{~cm}^{2}, \mathrm{p}<0.001$ ) were similar to AVA by PC-CMR and invasive AVA. Intra- and interobserver reproducibility were excellent for AVA (intraclass-correlation-coefficients of 0.939 and 0.827 , respectively).

Conclusion PC-CMR provides a great option to yield reliable and solid SV values in patients with moderate and severe AS. Furthermore, continuous determination of flow volumes and flow velocities is able to determine AVA in these patients in an easy and reproducible manner. Our novel approach shines a light on the diagnostic potential of PCCMR for non-invasive AS grading, especially in cases where echocardiography reaches its limits and where clinical findings appear inconclusive.
Image 1

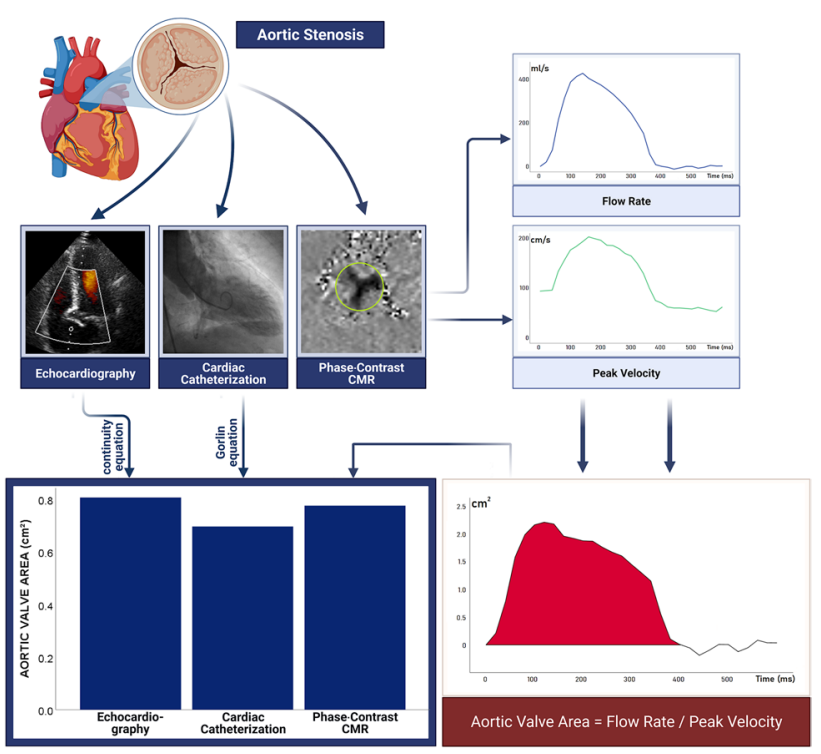

Image 2

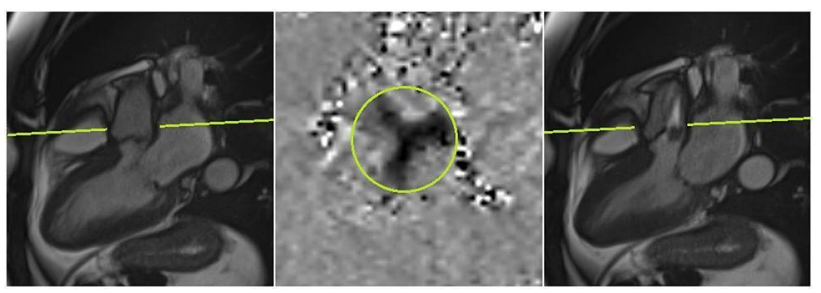




\section{Image 3}
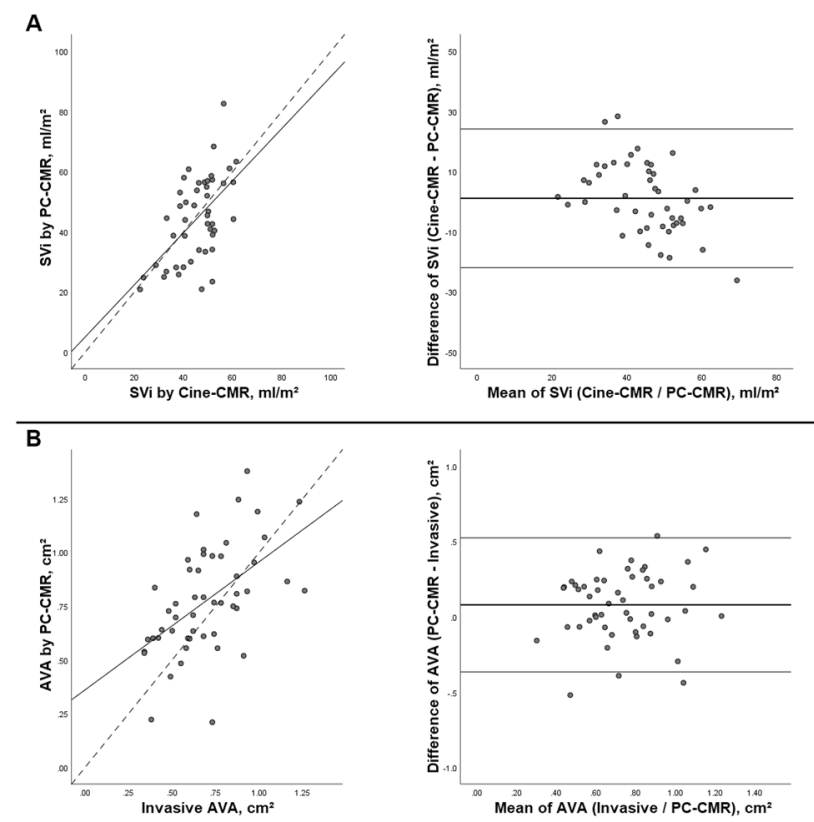

Presenter's first name and last name

Felix Troger

Presenter's city and country

Innsbruck, Tyrol, Austria

Presenter's email address

felix.troger@i-med.ac.at

\section{3}

\section{Long-term Evolution of Myocardial Tissue Injury after ST-Elevation Myocardial Infarction}

Felix Troger, Agnes Mayr, Gert Klug, Martin Reindl, Ivan Lechner, Christina Tiller, Magdalena Holzknecht, Mathias Pamminger, Felix Troger, Axel Bauer, Sebastian Reinstadler, Bernhard Metzler

Keywords Myocardial Infarction, STEMI, Cardiovascular Magnetic Resonance Imaging, Myocardial Tissue Injury

Topic Area of Interest

Purpose/Objectives In patients with first ST-elevation myocardial infarction (STEMI), the evolution of myocardial tissue injury parameters over a decade as assessed by cardiac magnetic resonance (CMR) has not yet been described. This study examined long-term myocardial tissue injury dynamics in STEMI patients treated with primary percutaneous coronary intervention (PCI), as well as its association with patient characteristics.

Methods and Materials Sequential CMR studies (after 3 days [interquartile ranges (IQR) 2-4], 4 months (m) [IQR 4-5] and 9 years (y) [IQR 8-10]) were conducted in a total of 104 STEMI patients to assess left ventricular (LV) dimensions and function, infarct size and microvascular obstruction (MVO). T2* mapping was added at $9 \mathrm{y}$ scan to assess the presence of iron within the infarct core. Results Infarct size decreased progressively from 13.3\% of LV myocardial mass [IQR $6.5-20.5$ ] to $10.2 \%$ [IQR 5.2-16.1] to 7.7\% [IQR 2.4-12.2] (all $\mathrm{p}<0.001$ ), with an average reduction rate of $6.3 \%$ per year [IQR 3.7-9] and a relative reduction of $43 \%$ [IQR 18-66] over a decade. MVO was present in 60\% (60/104) of patients at baseline, but in none of the follow-up examinations. Sixteen patients $(16 / 76,21 \%)$ had persistent iron within the infarct core at 9y CMR. Persistent iron was associated to a younger patient age $(\mathrm{p}=0.022)$, greater infarct size on any occasion (all $\mathrm{p}<0.01$ ) as well as presence of MVO ( $<<0.002)$. Patients with persistent iron showed a lower relative regression of infarct size (34\% [IQR 24-49] versus 53\% [IQR 22-70], $\mathrm{p}=0.037$ ) over a decade and greater endsystolic volumes on any occasion (all $\mathrm{p}<0.035)$.

Conclusion The involution of infarct size is a dynamic process that extends well beyond the first few months after STEMI. While MVO vanishes in the first few weeks, persistence of infarct iron occurs up to a decade after reperfused STEMI and is associated with initial infarct severity and worse infarct healing.

\section{Image 1}

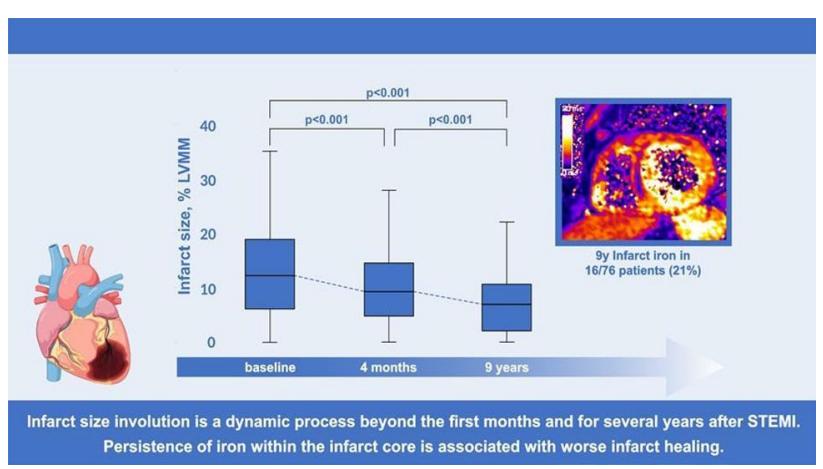




\section{Image 2}

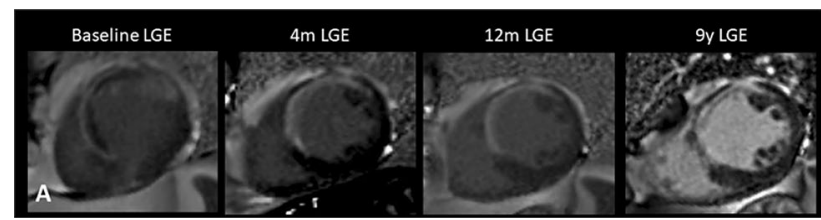

Presenter's first name and last name

Agnes Mayr

Presenter's city and country

Innsbruck, Tyrol, Austria

Presenter's email address

a.mayr@i-med.ac.at

\section{4}

\section{Pulmonary Transit Time by Cardiac Magnetic Resonance Imaging for Optimized Prognosis Assessment in ST-elevation Myocardial Infarction}

\section{Felix Troger, Martin Reindl, Mathias Pamminger, Christina Tiller, Magdalena Holzknecht, Ivan Lechner, Sebastian Reinstadler, Axel Bauer, Bernhard Metzler, Gert Klug, Agnes Mayr}

Keywords Myocardial Infarction, Transit-Time, Cardiovascular Magnetic Resonance Imaging, Infarct Severity

Topic Area of Interest

Purpose/Objectives Pulmonary transit time (pTT) may serve as surrogate parameter for cardiopulmonary haemodynamics and has been linked to heart failure. However, cardiac magnetic resonance (CMR) data on pTT and its associates with clinical outcome after ST-elevation myocardial infarction (STEMI) are lacking so far. We aimed to investigate the prognostic value of pTT determined by CMR in patients suffering from acute STEMI.

Methods and Materials Comprehensive CMR examinations were performed in 207 patients 3 (interquartile range (IQR) 2-4) days and 4 (IQR 4-5) months after reperfused STEMI for determination of functional parameters and infarct characteristics. PTT was measured as time between the peaks of time-intensity curves of gadolinium contrast to pass from the right ventricle (RV) to the left ventricle (LV) in first pass perfusion imaging. Primary endpoint was the occurrence of major adverse cardiac events (MACE) defined as composite of death, re-infarction and congestive heart failure.

Results PTT decreased significantly between baseline and 4-month CMR scan (8.6 (IQR 7.5-9.6) seconds (sec) to 7.8 (IQR 7-8.7) sec, $\mathrm{p}<0.001)$. According to Cox-regression ("functional model"), baseline pTT (hazard ratio [HR] 1.58, 95\% confidence interval [CI] 1.12-2.22; $p=0.009$ ) remained significantly associated to the occurrence of MACE after adjustment for LV ejection fraction (LVEF) and cardiac index. According to Cox-regression ("tissue model"), baseline pTT (HR 1.46, 95\% CI 1.02-2.10, p=0.039) as well as extent of microvascular obstruction (HR 1.20, 95\% CI $1.09-1.32, p=0.001)$ remained significantly associated to MACE after adjustment for infarct size. In Kaplan-Meier analysis, pTT $\geq 9.6 \mathrm{~s}$ (best cut-off) was associated with MACE $(\mathrm{p}<0.001)$. Addition of pTT to LVEF resulted in a categorical net reclassification improvement of $0.73(95 \%$ CI $0.27-1.20, \mathrm{p}=0.002$ ) and integrated discrimination improvement of 0.07 (95\% CI 0.02-0.13, $\mathrm{p}=0.007$ ).

Conclusion Following reperfused STEMI, CMR derived pTT predicts hard clinical events independent of infarct size and LV systolic function. Moreover, pTT provides additional prognostic value over LVEF alone.

\section{Image 1}

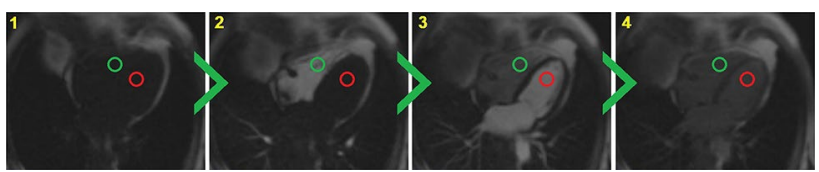

Image 2

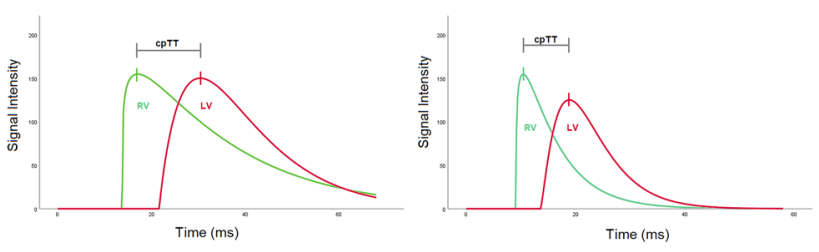

Presenter's first name and last name Felix Troger

Presenter's city and country Innsbruck, Tyrol, Austria 
Presenter's email address

felix.troger@i-med.ac.at

\section{5}

\section{Advanced cardiac magnetic resonance tools in characterizing Takotsubo syndrome: a pilot study}

\section{Riccardo Cau, Pierpaolo Bassareo, Jasjit Suri, Luca Saba}

Keywords Takotsubo, T1 mapping, T2 mapping, Myocardial Strain, Atrial strain

\section{Topic Area of Interest}

Purpose/Objectives The aims of our pilot study were to evaluate the application of atrial, ventricular myocardial strain and tissue mapping in characterizing Takotsubo syndrome (TS).

Methods and Materials Two groups were retrospectively enrolled: patients with Takotsubo syndrome $(n=19)$ and healthy controls $(n=11)$. We assessed global and regional left ventricular function, including longitudinal (LS), circumferential (CS), and radial strain (RS) analysis. Reservoir, conduit, and booster bi-atrial functions were analyzed by CMR-FT. Tissue characterization by T1, T2 mapping, and LGE was performed as well to detect the possible presence of myocardial injuries.

Results LS was reduced in patients with TS compared to healthy controls. LS dysfunction was detected mainly at mid- and apical cavity ( $\mathrm{p}=0.001$ for both). Again, basal RS was higher in TS patients compared to the control group. Regarding atrial strain, LA Reservoir strain ( $\varepsilon s)$, LA Reservoir strain rate (SRs), LA and RA Conduit strain(ee), LA and RA conduit strain rate (SRe) were impaired in comparison with controls $(\mathrm{P}<0.001$ for all of them). No other statistically significant differences in myocardial strain were detected. TS patients had higher global parametric mapping values, with greater involvement of apical segments $(\mathrm{p}=0,001)$.

Conclusion Our study suggests that left ventricular, bi-atrial myocardial strain impairment and parametric mappings could help in refining the evaluation of TS patients.

Presenter's first name and last name

Riccardo Cau

Presenter's city and country

Cagliari, Italy

Presenter's email address riccardocau00@gmail.com

\section{6}

\section{Advanced cardiac magnetic resonance tools in characterizing Myocarditis: a pilot study}

\section{Riccardo Cau, Valeria Cherchi, Michele Porcu, Jasjit Suri, Luca Saba}

Keywords Myocarditis, T1 mapping, T2 mapping, Myocardial strain, Atrial strain

Topic Area of Interest

Purpose/Objectives The aims of our pilot study were to evaluate the application of atrial, ventricular myocardial strain and tissue mapping in characterizing acute myocarditis (AM).

Methods and Materials Two groups were retrospectively enrolled: patients with acute myocarditis $(n=13)$ and healthy controls $(n=10)$. We assessed global and regional left ventricular function, including longitudinal (LS), circumferential (CS), and radial strain (RS) analysis. Regarding atrial function, reservoir, conduit, and booster bi-atrial functions were analyzed by CMR-FT. Tissue characterization by T1, T2 mapping, and LGE was performed as well to detect the possible presence of myocardial injuries.

Results All global ventricular strain parameters were reduced in patients with AM compared to healthy subjects. LS dysfunction was detected from basal to apical cavity $(\mathrm{p}=0,0001, \mathrm{p}=0,0001$ and $\mathrm{p}=0,03$, respectively). Again, apical RS and CS was lower in AM patients compared to the control group ( $p=0,018$ and $p=0,005$, respectively). No other statistically significant differences in myocardial strain were detected. Moreover, AM patients showed an impaired LA Reservoir strain ( $\varepsilon s)$, and RA Reservoir strain ( $\varepsilon s)$, in comparison with controls $(\mathrm{p}=0,04$ and $\mathrm{p}=0,02)$, while no differences were found as to others bi-atrial strain parameters. AM patients had higher global T2 values $(p=0,03)$, with higher regional values involving all left ventricle ( $p=0,01, p=0,04$ and $p=0,03$ from basal to apical segments). Global LS and apical CS proved to have an excellent performance in differentiating AM patients from controls (AUCs of 0.96 and 0.82 , respectively).

Conclusion Our study suggests that left ventricular, bi-atrial myocardial strain impairment and parametric mappings could help in refining the evaluation of AM patients.

Presenter's first name and last name

Riccardo Cau 
Presenter's city and country

Cagliari, Italy

Presenter's email address

riccardocau00@gmail.com

\section{7}

\section{Pulmonary transit time as a marker of diastolic dysfunction in Takotsubo}

\section{Riccardo Cau, Michele Porcu, Carlotta Onnis, Jasjit Suri, Luca Saba}

Keywords Pulmonary transit time, CMR, Takotsubo

Topic Area of Interest

Purpose/Objectives The exact pathophysiology of Takotsubo syndrome (TS) is not completely known, and the diastolic function is still an open issue. Pulmonary transit time (PTT) has been proposed as a useful marker for the evaluation of diastolic impairment. The aims of our pilot study were to evaluate the pulmonary transit time (PTT) by cardiac magnetic resonance (CRM) as a marker of diastolic dysfunction in Takotsubo syndrome (TS).

Methods and Materials Two groups were retrospectively enrolled: patients with TS $(\mathrm{n}=22$, age $68,95 \pm 9,2$, females $90 \%$ ) and healthy controls $(n=12$, age $47,33 \pm 20,2$, females $70 \%$ ). We assessed PTT by cardiac magnetic resonance (CMR). PTT has been calculated as the number of cardiac cycles, required for a bolus of contrast agents to move from right ventricle (RV) to left ventricle (LV).

Results In comparison with healthy subjects, TS patients showed significantly higher PPT $(8,7 \mathrm{~s} \pm 2,2$ vs $5,25 \mathrm{~s} \pm 1,8$, $\mathrm{p}=0,001$ ).In a multivariate analysis, there was a statistically significant difference between TS and controls regarding PPT after controlling for gender and age. PPT proved to have an excellent performance in differentiating TS patients from controls (AUCs of 0.90).

Conclusion PPT assessed by first-pass CMR is a potential marker for assessment left ventricle diastolic dysfunction in patients with TS.

Presenter's first name and last name

Riccardo Cau

Presenter's city and country

Cagliari, Italy

Presenter's email address

riccardocau00@gmail.com
0028

\section{Sensitivity and specificity of the measurement of epicardial adipose tissue thickness as a predictor of the severity of coronary artery disease}

\author{
Pawel Gac, Piotr Macek, Malgorzata Poreba, Rafal Poreba
}

Keywords CT/CT-Angiography, epicardial adipose tissue thickness, CAD-RADS

Topic Imaging Technique

Purpose/Objectives The aim of the study was to assess the usefulness of measuring epicardial adipose tissue thickness as a predictor of the severity of coronary artery disease (CAD).

Methods and Materials The study included 71 patients from cardiovascular outpatient clinics, who were referred based on the cardiovascular risk assessment and the diagnostics performed so far for a 128-row computed tomography angiography of the coronary arteries (coroCT) (age: $54.19 \pm 9.74$ years). The epicardial adipose tissue thickness (EATT) and the coronary artery disease severity were determined based on the coroCT images in all the patients. EATT was measured based on a multiplanar reconstruction, in the short axis of the left ventricle, at the level of the mid-ventricular layers, in the middle of the free wall of the right ventricle. The coronary artery disease severity was determined based on the CAD-RADS, where 0-documented absence of CAD, and 5-occlusion of at least one coronary branch. Results Mmean EATT value measured was $8.84 \pm 3.02 \mathrm{~mm}$. Based on CAD-RADS, $45.1 \%$ of the study group had documented absence of CAD (CAD-RADS 0, no stenosis), 25.3\% - minimal CAD (CAD-RADS 1, 1-24\% stenosis), 15.5\%- mild CAD (CAD-RADS 2, 25-49\% stenosis), 7.0\%-moderate CAD (CAD-RADS 3, 50-69\% stenosis), 5.6\%- severe CAD (CAD-RADS 4, stenosis 70-99\%), and $1.4 \%$ - coronary artery occlusion (CAD-RADS $5,100 \%$ stenosis). Subgroup with documented absence of CAD (CADRADS 0) had statistically significantly lower EATT than subgroup with coronary lesions (CAD-RADS 1-5). Correlation analysis showed the statistically significant positive correlation between EATT and CAD-RADS $(r=0.25)$. Based on the ROC curve, the EATT value was determined, which is the optimal cut-off point for the prediction of documented absence of CAD in the study group of patients (CAD-RADS 0 ), amounting to $9.6 \mathrm{~mm}$. The sensitivity, specificity, and accuracy of EATT $<9.6 \mathrm{~mm}$ as a CAD-RADS 0 predictor were $0.514,0.800$, and 0.642 , respectively. 
Conclusion A coroCT epicardial adipose tissue thickness of less than $9.6 \mathrm{~mm}$ may be a good predictor of documented absence of coronary artery disease.

Presenter's first name and last name

Pawel Gac

Presenter's city and country

Wroclaw / Poland

Presenter's email address.

pawelgac@interia.pl

\section{9}

\section{Coronary arteries pseudoaneurysms after drug-eluting stent implantation: role of coronary CT angiography}

\section{Serena Dell'Aversana, Serena Dell'Aversana, Giuseppe Di Costanzo, Luigi Palumbo, Marta Puglia, Ilaria Ferrara, Giovanna Gernone, Fabio Acanfora, Marco Boccalatte, Vito Di Palma, Gerolamo Sibilio, Bianca Cusati.}

Keywords Coronary artery disease, Atherosclerosis, Aneurysm

\section{Topic Special Focus}

Purpose/Objectives Coronary pseudoaneurysm (CA) is an uncommon but potentially life-threatening complication with an incidence of $0.2 \%-2.3 \%$ after drug-eluting stent (DES) implantation. More recently, with the increasing use of drug-eluting stents (DESs), there are growing reports signifying stents causing coronary aneurysms, months or even years after the intervention. Dilatation may be either focal or diffuse, and aneurysms are classified as either fusiform (longitudinal dimension $>$ transverse dimension) or saccular (transverse dimension $>$ longitudinal dimension) in morphology.

Methods and Materials The pathophysiology of CA still remains unclear but is perceived to be identical to that for aneurysms of larger vessels, destruction of arterial media, thinning of the arterial wall, increased wall stress, and progressive dilatation of the coronary artery segment. Several features unique to DES have been proposed to contribute to the formation of CA after implantation. Type I DES-CA occur immediately (within one month) of stent placement. Pathophysiology is similar to that seen in early reports involving BMS with arterial injury from mechanical factors such as stretching, stent fracture, dissection, haematoma. Type II DES-CA occurs sub-acutely (6-9 months) and is secondary to arterial wall response to the DES. Type III DESCA refers to infectious mycotic-aneurysms. Both sirolimus and paclitaxel are potent anti-mitotic agents which inhibit cell-cycle progression and thereby inhibit neointimal hyperplasia. However, it is this same antiproliferative effect which has been proposed as increasing the risk of CA.

Results Coronary angiography remains the gold standard tool as it provides information about the shape, size, location, and coexisting anomalies such as $\mathrm{CAD}$, and is also useful for setting up the strategy of surgical resection. Among noninvasive modalities, coronary $\mathrm{CT}$ is an alternative to invasive coronary angiography that can be suggested as a technique of choice due to the high anatomical definition, plays an important role in the follow-up of patients with CA because of improvements in terms of radiation dose with the current protocols.

Conclusion Discrimination between true aneurysm and pseudoaneurysm and the detection of the aneurysm entry site are important for optimal management and coronary $\mathrm{CT}$ proved to be an accurate non-invasive tool. There is no standard therapy for coronary aneurysms but coronary CT improves the treatment planning.

Presenter's first name and last name

Serena Dell'Aversana

Presenter's city and country

Pozzuoli (Na), Italy

Presenter's email address

dellaversanaserena@gmail.com

\section{0}

An easy recipe for performing 80-kV ECG-gated high-pitch aortic CT angiography with a peristaltic contrast media injector

\section{Hug Cuellar-Calabria, Anton Aubanell Creus, Albert Roque Pérez, Gemma Burcet, Jose L Reyes-Juarez}

Keywords CT/CT-Angiography, Aortic disease, ECG-gated high-pitch CT

Topic Imaging Technique

Purpose/Objectives To design a contrast media (CM) injection protocol for high-pitch CT angiography of the thoracoabdominal aorta on a peristaltic injector system using weight-based CM dilution and a double-level test bolus for calculation of CM volume and scan delay. To analyze 
the overall and intraindividual image quality of the new protocol.

Methods and Materials 50 patients underwent an $80-\mathrm{kV}$ ECG-gated high-pitch CT angiography of the thoracoabdominal aorta ( $70 \pm 11$ years). CM volume, injection duration and scan delay were tailored on a peristaltic injector system according to the results of a double-level test bolus. Iohexol $350 \mathrm{mgI} / \mathrm{ml}$ was diluted for groups $\mathrm{M}(175 \mathrm{mgI} /$ $\mathrm{ml}, 50-75 \mathrm{~kg}, \mathrm{n}=19), \mathrm{L}(233 \mathrm{mgI} / \mathrm{ml}, 76-100 \mathrm{~kg}, \mathrm{n}=24)$ and XL (350 mgI/ml, > $100 \mathrm{~kg}, \mathrm{n}=7)$. Weight, height and dose-length product (DLP) were recorded for each patient. Attenuation, coefficients of variation (CV) and signal-tonoise ratio (SNR) were obtained in the superior vena cava (SVC), main pulmonary artery (MPA), five aortic locations and femoral arteries. ANOVA and t-tests were used to check to compare attenuation among vessels and to analyze homogeneity of attenuation and SNR across aortic locations and among weight groups. Automatically-rendered volumetric images were reviewed and incomplete aortic visualization due to interposition of enhanced vessels was scored on a semiquantitative scale.

Results Mean weight and DLP were $83 \pm 18 \mathrm{~kg}$ and $175 \pm 18 \mathrm{mGy}^{*} \mathrm{~cm}$, respectively. Mean peak circulation time, iodine delivery rate, $\mathrm{CM}$ volume and Iodine amount were $11 \pm 5 \mathrm{~s}, 11 \pm 1.1 \mathrm{mg} \mathrm{I} / \mathrm{kg} / \mathrm{s}, 47 \pm 15 \mathrm{ml}$ and $16.4 \pm 5.2 \mathrm{~g}$, respectively. Overall, mean aortic attenuation was $396 \pm 88 \mathrm{HU}$ (>250 HU in $98 \%$ of patients), higher than in the SVC and MPA ( $184 \pm 98$ and $144 \pm 125$ $\mathrm{HU}$, respectively; $\mathrm{p}<0.001)$. Intrapatient aortic attenuation showed a non-significant $8 \% \mathrm{CV}(\mathrm{p}=0.07)$. Group $\mathrm{L}$ showed higher attenuation than group M $(426 \pm 95 \mathrm{HU}$ versus $345 \pm 55 \mathrm{HU}$, respectively; $\mathrm{p}=0.001$ ) but similar SNR $(26.1 \pm 6.2$ versus $24.4 \pm 5.4$, respectively; $p=0.35)$. Complete aortic visualization in volume-rendered images was achieved in $92 \%$ of patients.

Conclusion A mean aortic attenuation of $396 \pm 88 \mathrm{HU}$ with no significant intrapatient variation across aortic locations was achieved at 80-kV ECG-gated high-pitch aortic CT angiography in a standard European population after an individually tailored CM injection using a peristaltic injector system. Complete aortic visualization without obstructing vessels or organs was achieved in automatically-rendered volumetric images of $92 \%$ of patients. Mean CM volume and DLP were consistently low ( $47 \pm 15 \mathrm{ml}$ and $175 \pm 18 \mathrm{mGy} * \mathrm{~cm}$, respectively).

\section{Image 1}

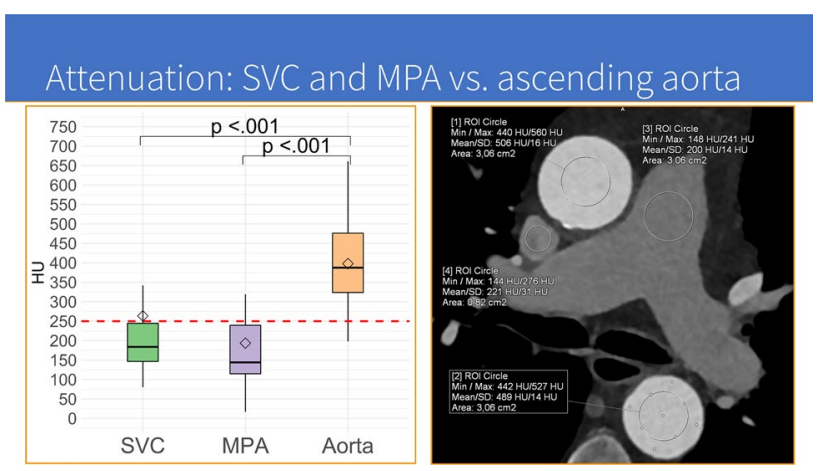

Image 2

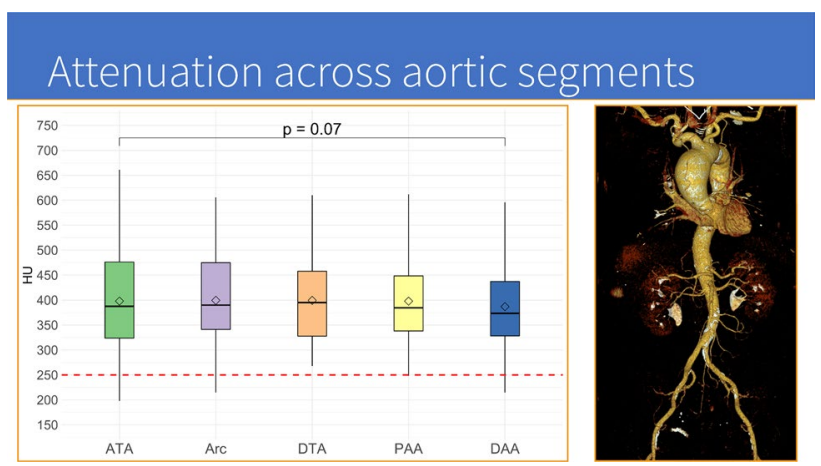

Image 3

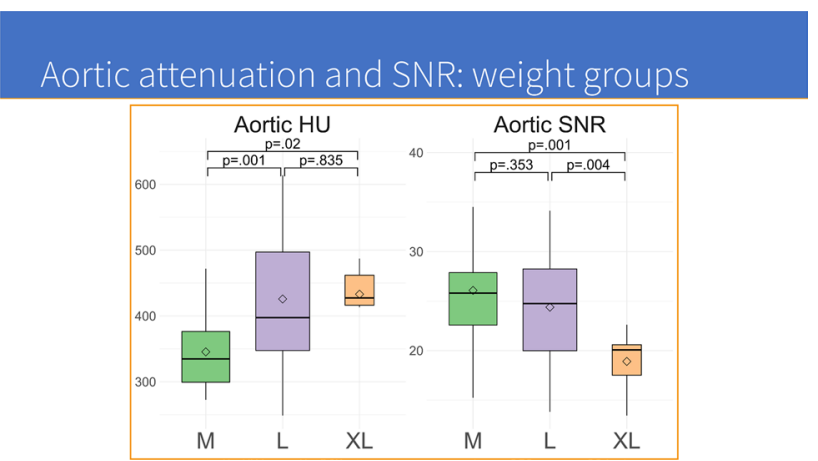


Presenter's first name and last name

Hug Cuellar-Calabria

Presenter's city and country

Barcelona, Spain

Presenter's email address

hug.cuellar.idi@gencat.cat

\section{1}

\section{Radiation exposure for patients with stable chest pain in the SCOT-HEART study}

\section{Justin Chiong, Prasad Guntur, Jonathan Weir-McCall, Alberto Nania, Marc Dweck, Nicholas L Mills, Edwin J van Beek, Giles Roditi, David E Newby, Michelle Williams}

Keywords Computer Tomography, Radiation Dose, SCOT-HEART

\section{Topic Imaging Technique}

Purpose/Objectives Computed tomography coronary angiography (CCTA) is central to many diagnostic strategies for suspected coronary artery disease (1). However, radiation exposure remains an important concern in cardiovascular imaging due to the potential lifetime risks of cancer (2). In Scottish Computed Tomography of the Heart (SCOT-HEART) trial, patients with suspected angina due to coronary artery disease were randomised to undergo either standard care or standard care plus CT. We showed that management based on CCTA altered the use of subsequent investigations and preventative medications, and led to improved patient outcomes $(3,4)$. After five years, the use of invasive coronary angiography (ICA) and revascularisation was similar between CT and standard care groups (4). However, the long-term cumulative radiation exposure in this population has not been assessed. This study aims to assess the five-year cumulative radiation dose from cardiac imaging for patients randomised to CT or standard care groups of the SCOT-HEART trial.

Methods and Materials We included 2894 patients from the SCOT-HEART trial (1466 standard care, 1428 CT group) from 6 recruiting centres where information on radiation doses was available. Radiation doses were assessed from CT (dose length product, DLP), single photon emission tomography (SPECT; megabecquerel, $\mathrm{MBq}$, of injected activity) and invasive coronary angiography (ICA; dose area product, DAP). Effective radiation dose for CT was calculated from DLP using the currently recommended conversion factor $(0.014 \mathrm{mSv} / \mathrm{mGy} . \mathrm{cm})$ and a conversion factor for cardiac
CT (0.026 mSv/mGy.cm) (5). This included follow-up imaging to assess incidental lung nodules picked up on scans, recommended for $7.6 \%$ of trial patients (6). SPECT effective radiation dose was calculated by multiplying by a conversion factor $(0.0073 \mathrm{mSv} / \mathrm{MBq}$ for rest SPECT, $0.0065 \mathrm{mSv} /$ $\mathrm{MBq}$ for stress SPECT) and invasive coronary angiography from DAP using the $0.0024 \mathrm{mSv} / \mathrm{cGy} . \mathrm{cm} 2$ conversion factor. Results Mean age was $57 \pm 10$ years and $57 \%$ were male. Median DLP for calcium score was 116 [IQR 99, 126] mGycm and CCTA was 158 [100, 234] mGycm. Median injected activity was $699[639,765] \mathrm{MBq}$ for rest and 627 [346, 712] MBq for stress SPECT. Median DAP was 1869 [1291, 2801] cCycm2 for diagnostic ICA and 4242 [2940, 6297] cCycm 2 for intervention. After 5 years, cumulative dose was lower in the standard care $(0.0[0.0,6.6]$ $\mathrm{mSv})$ compared to the CT group $(4.5[3.0,8.3] \mathrm{mSv}$ using $0.014 \mathrm{mSv} / \mathrm{mGy} . \mathrm{cm} ; 8.0[5.5,12.7] \mathrm{mSv}$ using $0.026 \mathrm{mSv} /$ mGy.cm; $\mathrm{p}<0.001$ ). No imaging was performed in $9 \%$ in the CT group and $63 \%$ in the standard care group. For patients undergoing imaging, cumulative dose was higher in the standard care $(8.6[5.1,11.6] \mathrm{mSv})$ than the CT group $(4.8$ [3.3 to 9.1] $\mathrm{mSv}$ using $0.014 \mathrm{mSv} / \mathrm{mGy} . \mathrm{cm} ; 8.5$ [6.1, 13.4] $\mathrm{mSv}$ using $0.026 \mathrm{mSv} / \mathrm{mGy} . \mathrm{cm} ; \mathrm{p}<0.001$ ).

Conclusion In this study we assessed cumulative radiation dose triggered by clinic attendance for suspected angina due to coronary heart disease. This included cardiac CT, nuclear perfusion, invasive coronary angiography, and CT for lung nodule follow-up. Overall, the 5-year cumulative radiation dose was low in both groups. When assessing all patients, total radiation dose was higher in the CT group compared to the standard care group, with this difference primarily due to the radiation dose of cardiac CT. However, when considering those who underwent imaging (91\% in the CT group, $37 \%$ in the standard care group), radiation dose was lower in the CT group compared to the standard care group. Nevertheless, it must be remembered that the CT group had significantly improved outcomes, with a $50 \%$ reduction in fatal or non-fatal myocardial infarction. Therefore, a diagnostic strategy based on CT is a valuable option for patients presenting with stable chest pain.

\section{References}

ESC Guidelines for the diagnosis and management of chronic coronary syndromes: The Task Force for the diagnosis and management of chronic coronary syndromes of the European Society of Cardiology. European Heart Journal 2019.

Huda W et al. Radiation-related cancer risks in a clinical patient population undergoing cardiac CT. AJR Am J Roentgenol 2011;196(2):W159-165.

Scot-Heart Investigators. CT coronary angiography in patients with suspected angina due to coronary heart disease 
(SCOT-HEART): an open-label, parallel-group, multicentre trial. Lancet 2015;385(9985):2383-2391.

Scot-Heart Investigators. Coronary CT Angiography and 5-Year Risk of Myocardial Infarction. N Engl J Med 2018;379(10):924-933.

Trattner S et al. Cardiac-Specific Conversion Factors to Estimate Radiation Effective Dose From Dose-Length Product in Computed Tomography. JACC Cardiovasc Imaging 2018;11(1):64-74.

Williams MC et al. Impact of noncardiac findings in patients undergoing CT coronary angiography: a substudy of the SCOT-HEART trial. Eur Radiol 2018;28(6):2639-2646.

\section{Image 1}

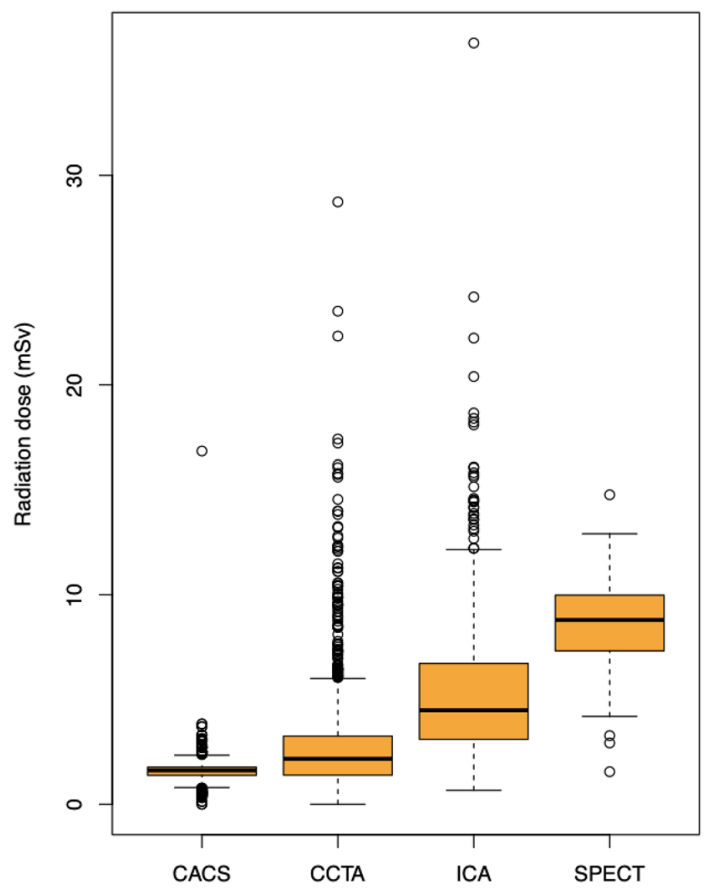

Image 2

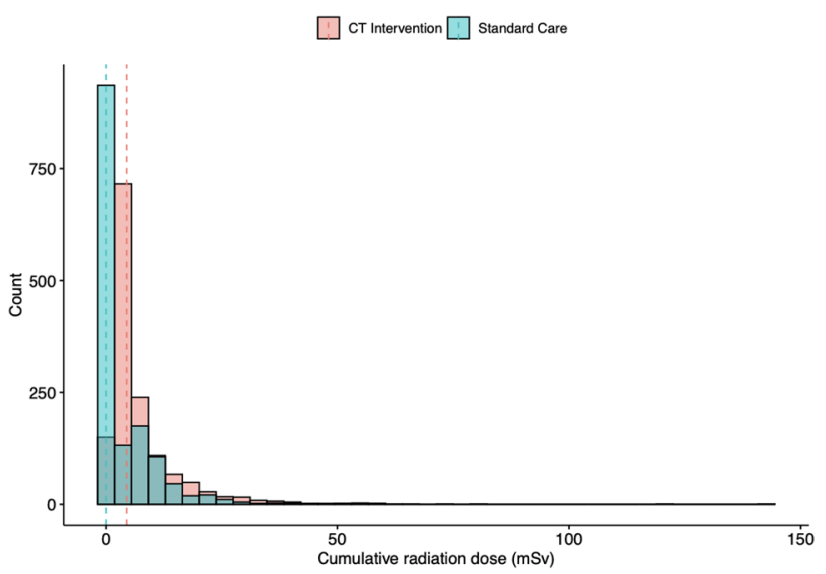

Presenter's first name and last name Justin Chiong

Presenter's city and country

Edinburgh, United Kingdom

Presenter's email address

justin.wei@nhs.scot

\section{2}

Pre-TAVI aortic annulus sizing: Comparison between manual and semi-automated new generation software measurements

Andrea Daniele Annoni, Aurora Ruffino, Roberta Colombo, Giuseppe Muscogiuri, Maria Elisabetta Mancini, Alberto Formenti, Carolina Lanza, Daniele Andreini, Gianluca Pontone

Keywords CT/CT-Angiography, TAVI, CT software 


\section{Topic Imaging Technique}

Purpose/Objectives TAVI Planning requires important information and CT is used to help determine aortic annulus size, to guide selection of appropriate valve, provide dimensions of the entire aorta and give guidance for valve deployment. This requires accurate and time-consuming manual measurements performed by experienced operators. Semi-automatic software can aid the CT reconstructions reducing the time needed for multiple measurements assessment and improving measurements reproducibility. Aim of this study is to compare manual and semi-automatic measurements for aortic annulus assessment in terms of accuracy, agreement and time among different operators with different experience in cardiovascular CT reconstruction.

Methods and Materials 80 patients that underwent successful TAVI with pre procedural CT were retrospectively evaluated from our internal database. These patients related measurements previously manually performed by an experienced reader ( $>12$ years of experience in cardiovascular CT) for aortic annulus dimensions (minimum and maximum diameters, perimeter, area), distance from the annulus to the coronary ostia and the time needed for the whole evaluation, were collected and considered as standard reference because confirmed by the final procedure. The same operator (observer 1) assessed the same measurements in the same patients using a semi-automatic software. The same measurements were performed by two less experienced readers (observer 2 and observer 3 , with $>5$ years and 1 year of experience respectively) blinded to each other's results. Differences between manual and semi-automatic measurements and the reading time were compared. The suggested valves size derived by $\mathrm{CT}$ area were compared with the implanted prosthesis size. Results Very good correlations were found between manual and software aided measurements for aortic annulus area and perimeter for the 3 readers $(\mathrm{k}=0.98,0.9$ and 0.84 respectively). Good correlations were also found for the distance with coronary ostia. Good correlation for Intra observer annulus measurements were found ( $\mathrm{k}$ range 0.68-0.0.92). The same area-derived prosthesis size for manual and semi-automatic measurements was selected in $96 \%$ of cases for observer 1 . Very good correlations were found in area-derived prosthesis also for observer 2 and 3. The mean time needed for CT images reconstruction using manual measurements was recorded as $3.1 \mathrm{~min}$. Using semi-automatic measurements time recorded was significantly lower for observer 1 and 2 (1.5 and $1.7 \mathrm{~min}$ respectively) and slightly lower for observer 3 (2.9 min). Conclusion Pre-TAVI CT using semi-automatic software allows accurate and reproducible measurements, reducing the time needed for reconstructions up to $50 \%$ in comparison with the standard reformat-tool. Furthermore, semi-automatic software is feasible even for operators with varying degrees of experience.

\section{Image 1}

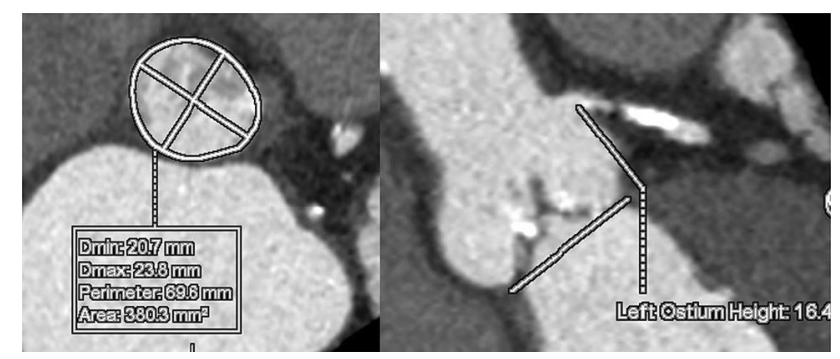

Image 2
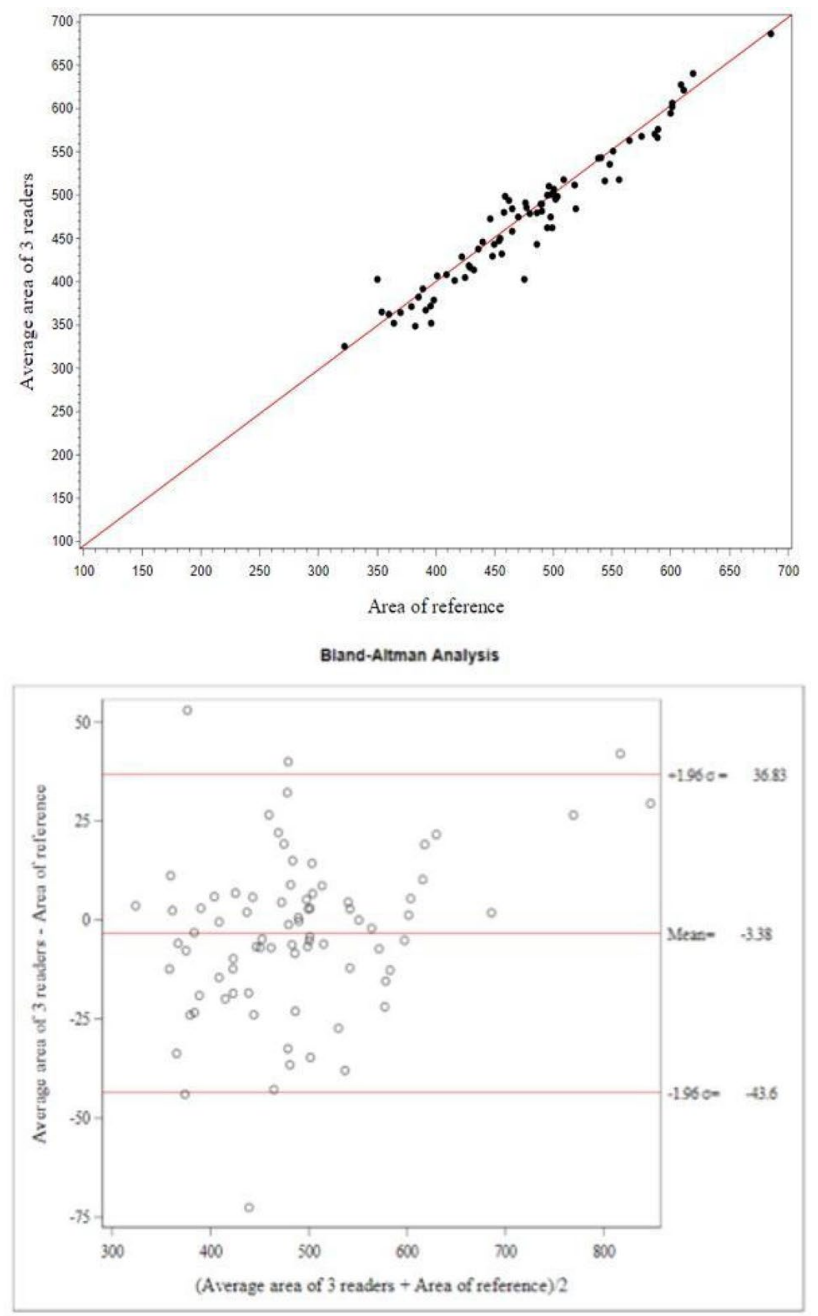
Presenter's first name and last name

Aurora Ruffino

Presenter's city and country

Milan, Italy

Presenter's email address

aurora.ruffino@unimi.it

\section{3}

\section{Al guided decision assistance for detecting cardiomegaly from chest $\mathrm{X}$-ray images in situations of cardiac surgery and intensive care}

\section{Meinrad Beer, Hanna Nieberler, Patrick Thiam, Ludwig Lauser, Christopher Kloth, Hagen Gorki, Andreas Liebold, Hans Kestler}

Keywords Artificial intelligence, Screening, Risk stratification

\section{Topic Procedure}

Purpose/Objectives To develop Deep Neural Networks (DNN) for detecting cardiomegaly in chest $\mathrm{x}$-ray images of patients prior and post cardiac surgery for faster clinical decisions in the setting of intensive care units. DNNs might help to support the diagnosis of heart failure by an integrated analysis of chest X-ray images and other clinical features (e.g. age, echocardiography results).

Methods and Materials This single center retrospective analysis consecutively included 129 patients (29 female and 100 male; mean age $65.5 \pm 11.9$ years, range $27-87$ years), who had chest radiography examinations prior and after cardiac surgery. Two radiologists verified and relabeled a total of 52 "normal" and 77 "cardiomegaly" images. Among the "cardiomegaly" images were 57 male (44.1\%) and 20 female (15.5\%) patients labeled. For "normal" 43 male $(33.3 \%)$ and 9 female $(7.1 \%)$ patients were labeled. "Cardiomegaly" was defined by a cardiothoracic ratio $>0.55$. As gold standard every patient received echocardiography and all patients underwent chest-x ray for follow-up.

Results For analysis two different DNN architectures (VGG16 and VGG19) with three different approaches were used. The best performing model (VGGXX Chest Xray
Model Cardiomegaly Data Augmentation) incorporated additional data (cardiomegaly $\mathrm{y} / \mathrm{n}$ ).

Sensitivity/ Specificity/ were 92.4 and 71.1\% (VGG16). Focussed data augmentation (cardiomegaly yes/no) was superior to mere data augmentation or training restricted to mere own data. F1-score, prediction and recall were significantly improved compared to unfocussed data augmentation or standard data.

Conclusion Deep learning shows a high promise to improved diagnosis of heart failure on the basis of chest $\mathrm{X}$-ray images in the setting of ICU imaging. Data augmentation seems to be promising approaches. Inclusion of additional data (echocardiography) might further improve detection rates.

\section{References}

Xiaosong Wang; Yifan Peng; Le Lu; Zhiyong Lu; Mohammadhadi Bagheri; Ronald M. Summers. ChestX-Ray8: Hospital-Scale Chest X-Ray Database and Benchmarks on Weakly-Supervised Classification and Localization of Common Thorax Diseases. 2017 IEEE Conference on Computer Vision and Pattern Recognition (CVPR). https:// doi.org/10.1109/CVPR.2017.369

Image 1

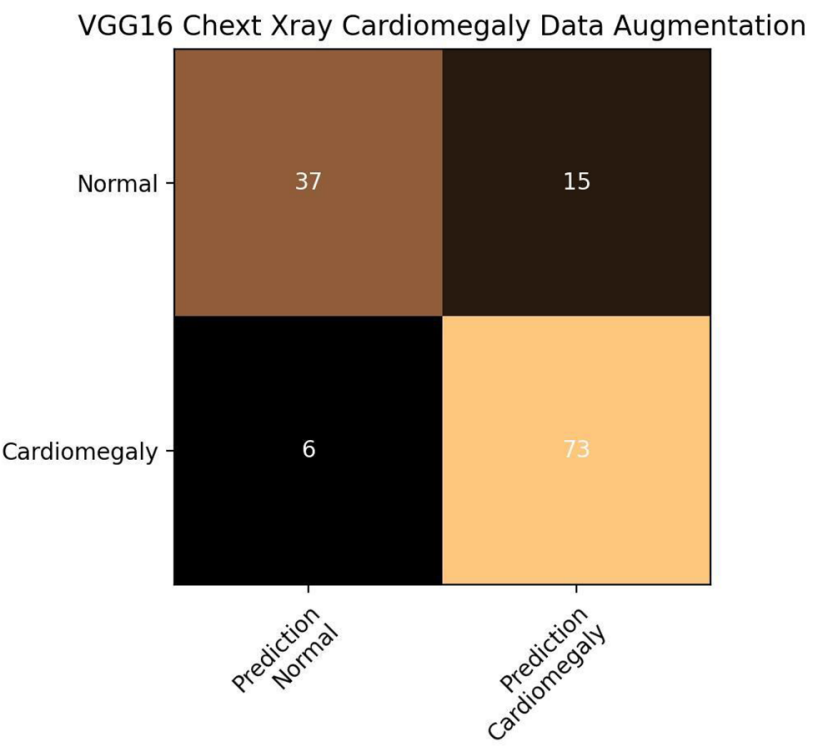




\section{Image 2}

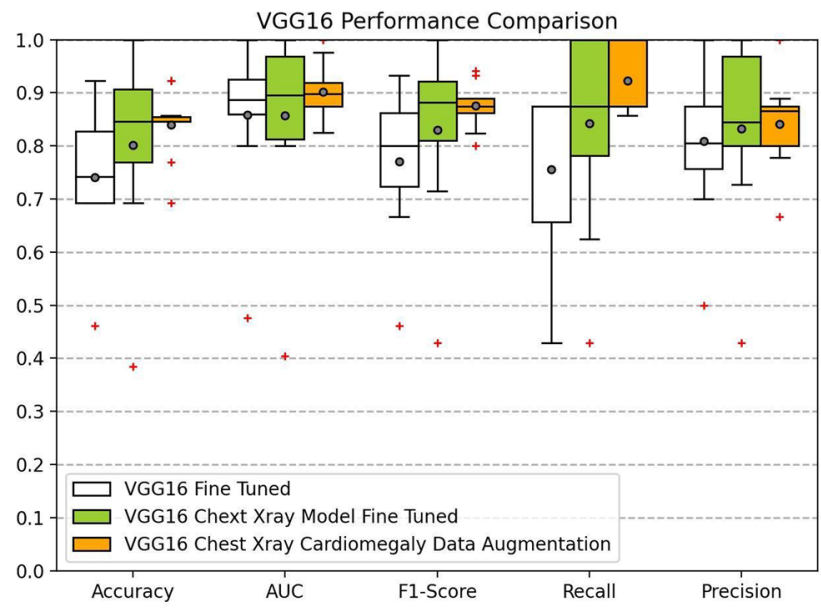

Presenter's first name and last name

Meinrad Beer

Presenter's city and country

Ulm Germany

Presenter's email address

meinrad.beer@uniklinik-ulm.de

\section{6}

\section{Evaluation of automated coronary calcium distribution in the Robinsca trial}

\section{Marleen Vonder, Sunyi Zheng, Carlijn van der Aalst, Harry de Koning, Dirkjan Kuijpers, Matthijs Oudkerk}

Keywords deep learning, coronary artery calcium, vessel distribution

\section{Topic Imaging Technique}

Purpose/Objectives Recent studies have shown that the coronary artery calcium (CAC) score can be improved by addition of CAC distribution, which is especially relevant in Agatston score 1-300.(1-3) Scoring of CAC is a highly repetitive and labour intensive task for radiologists. Workload is expected to increase drastically in case of population based screening. However, determining the number of affected vessels can be automated with the help of a deep learning based algorithm. Nevertheless, so far CAC distribution has not been taken into account in population based cardiovascular screening. The aim of this study was to compare automatic CAC distribution with manual assessed distribution and risk categorization in the Dutch populationbased cardiovascular screening trial (ROBINSCA).

Methods and Materials In total, 997 Robinsca participants (asymptomatic men (45-74 years), women (55-74 years) with an expected high risk for developing CHD) were included who were prospectively screened with ECGtriggered non-contrast dual-source CT (Somatom Flash, Siemens Healthineers, Germany) between December 2015 and June 2016, and informed consent was obtained from all participants.(4-6) The Agatston score and CAC distribution by means of the number of affected coronary arteries were determined with semi-automatic software manually (Syngo.via CaSc version VB10A, Siemens, Germany) and fully automatic by deep learning based AI software (AVIEW CAC, v1.0, Coreline Soft, Seoul, Korea). Agatston score based risk categorization $(0,1-100,101-399, \geq 400)$ was performed, and AI CAC distribution was analyzed per risk category. Spearman's correlation coefficients were calculated to determine relation between number of affected arteries (based on AI) and age, (manual) calcium score, (manual) risk categories. Distribution was compared between AI and manual based assessment using Wilcoxon signed rank test. Results In total 59.6\% (594) of the 997 participants (mean age 61.0 (SD 7.4), 54.4\% male) had a positive Agatston score. A slightly higher number of vessels were affected based on deep learning assessment (median: 1, mean: 1.57) compared to manual assessment (median:1 mean:1.30) $(\mathrm{p}<0.001)$. The number of affected arteries (based on AI) increased significantly for increasing age $(\mathrm{rho}=0.255)$, increasing calcium score $(\rho=0.878)$ and higher risk categories $(r h o=0.853)(\mathrm{p}<0.001)$. The probability of having a calcium score of $\geq 100$ for participants with $0,1,2,3$ or 4 vessels affected (based on AI) was 0\%, 4\%, 17.8\%, 49.7\% and $83.0 \%$ respectively. Whereas the probability of having a 0 -score for participants with $0,1,23$ or 4 , vessels affected (based on AI) was 94.9\%, 37.8\%, 6.5\% 0\% and 0\% respectively. Most heterogeneity in CAC risk group and CAC distribution, was seen for CAC score of 1-400.

Conclusion The number of affected vessels based on AI shows a high correlation with manually assessed calcium score and risk categorization. A considerable percentage of participants with 1 or 2 affected vessels are at high CVD risk category, but also a considerable percentage had a 0 -score. Therefore, CAC distribution may be used in addition to CAC calcium score in a cardiovascular screening setting. This CAC distribution will be most relevant in participants with CAC score between 1-400 to further improve risk stratification. 


\section{References}

Blaha MJ et al. Improving the CAC Score by Addition of Regional Measures of Calcium Distribution. JACC Cardiovasc. Imaging 2016;9:1407-1416.

Hecht HSet al. CAC-DRS: Coronary Artery Calcium Data and Reporting System. An expert consensus document of the SCCT. J.Cardiovasc.Comput.Tomogr. 2018;12:185-191. Dzaye $\mathrm{O}$ et al. Validation of the Coronary Artery Calcium Data and Reporting System (CAC-DRS): Dual importance of CAC score and CAC distribution from the Coronary Artery Calcium (CAC) consortium. J.Cardiovasc. Comput. Tomogr.2020;14:12-17.

Aalst CM Van Der et al. Risk or Benefit in Screening for Cardiovascular Disease (ROBINSCA): The Rationale and Study Design of a Population-Based Randomized-Controlled Screening Trial for Cardiovascular Disease. J.Clin. Trials2019;9:1-8.

van der Aalst CM et al. Screening for cardiovascular disease risk using traditional risk factor assessment or coronary artery calcium scoring: the ROBINSCA trial. Eur. Hear. J. -Cardiovasc.Imaging2020;21:1216-1224.

Vonder $\mathrm{M}$ et al. Coronary Artery Calcium Imaging in the ROBINSCA Trial. Acad.Radiol.2018;25:118-128.

Presenter's first name and last name

Marleen Vonder

Presenter's city and country

Groningen, Netherlands

Presenter's email address

m.vonder@umcg.nl

\section{7}

\section{Exploring Patient Experience and Preference of Investigations for Coronary Artery Disease}

\section{Elspeth McLaughlin, Danielle Richardson, Edwin J van Beek, David E Newby, Michelle Williams}

Keywords patient experience, computed tomography, coronary artery disease

\section{Topic Imaging Technique}

Purpose/Objectives Diagnostic tests for patients with suspected or known coronary artery disease include both invasive and non-invasive methods. These have been investigated extensively for diagnostic accuracy and clinical utility, but little is known about patient experience or preference. Understanding patient experience is crucial for improved patient counselling, patient satisfaction, investigation uptake and widespread acceptance of imaging techniques in clinical practice. A small number of previous studies have assessed patient preference in cardiac imagining. In anatomical imaging, computed tomography (CT) coronary angiography (CCTA) was preferred to magnetic resonance (MRI) angiography [1]. In a small study of perfusion imaging, CT perfusion (CTP) was preferred to MRI perfusion, single photon emission tomography (SPECT), and invasive coronary angiography (ICA) [2]. No studies have assessed patient experience of cardiac positron emission tomography (PET). Therefore, this study aims to assess patient experience of stress perfusion CT, MRI, oxygen-15 water PET, and ICA with fractional flow reserve (FFR).

Methods and Materials Patients with suspected or known coronary artery disease who were awaiting ICA were recruited. All patients underwent a comprehensive cardiac CT protocol including calcium score, CCTA, adenosine stress CTP and viability. A subset of patients underwent adenosine stress cardiac MRI perfusion and adenosine stress oxygen-15 water PET. ICA was performed with FFR assessment for major epicardial vessels with stenosis $>50 \%$, where technically possible [3]. After each imaging test, patients were asked to complete a self-administered questionnaire. The questionnaire asked patients to rate their concern, comfort, helplessness, and overall test satisfaction on a 5-point Likert scale. Patients reported pain during and after investigations was on a visual analogue scale. Overall preference was documented as well as free text information on their experience. Statistical analysis was performed on IBM SPSS V26. Two-tailed $\mathrm{P}$ values of $<0.05$ were considered significant and displayed as $* \mathrm{p}<0.05, * * \mathrm{p}<0.01, * * * \mathrm{p}<0.001$ on the Figures.

Results 48 patients (mean age $63 \pm 8,81 \%$ male) underwent $\mathrm{CT}$ and of these 44 had ICA, $30 \mathrm{MRI}$ and 22 PET. Patients reported less concern prior to CT compared to ICA, MRI or PET ( $<<0.01$, Fig. 1) and less helpless during CT and PET compared to ICA and MRI ( $p<0.05$, Fig. 2). Pain experienced during procedure was similar for all investigations ( $>0.05$, Fig. 3). However, post-procedure pain was greater with ICA than CT or MRI ( $p<0.001$, Fig. 3). Patients with coronary artery disease on CT experienced more pain during all imagining modalities compared to patients without ( $p<0.05$ for all). Comfort, overall satisfaction, and willingness to undergo each investigation again in the future were highly rated for all imaging modalities. Of patients indicating a preference, $58 \%$ preferred CT followed by $29 \%$ ICA and $10 \%$ MRI (Fig. 4). Reasons highlighted for this included the non-invasive, non-claustrophobic, fast and comfortable nature of CT. 
Conclusion In this study we found that during investigation for coronary artery disease, patients report a high degree of satisfaction, comfort and willingness to undergo each test again in the future for all imaging modalities. Patients experience less concern and helplessness during non-invasive imaging and more concern and pain postprocedure with ICA. The preferred imaging modality was CT, followed by ICA and then MRI. This information will be helpful for discussions between patients and physicians regarding choice of imaging tests for coronary artery disease and enable improvements in patient satisfaction, autonomy and investigation uptake.

\section{References}

Dewey M, Teige F, Schnapauff D, Laule M, Borges A, Wernecke K et al. Noninvasive Detection of Coronary Artery Stenoses with Multislice Computed Tomography or Magnetic Resonance Imaging. Annals of Internal Medicine. 2006;145(6):407.

Feger S, Rief M, Zimmermann E, Richter F, Roehle R, Dewey $\mathrm{M}$ et al. Patient satisfaction with coronary $\mathrm{CT}$ angiography, myocardial CT perfusion, myocardial perfusion MRI, SPECT myocardial perfusion imaging and conventional coronary angiography. European Radiology. 2015;25(7):2115-2124.

Williams M, Mirsadraee S, Dweck M, Weir N, Fletcher A, Lucatelli $\mathrm{C}$ et al. Computed tomography myocardial perfusion vs $15 \mathrm{O}$-water positron emission tomography and fractional flow reserve. European Radiology. 2016;27(3):1114-1124.3.

\section{Image 1}

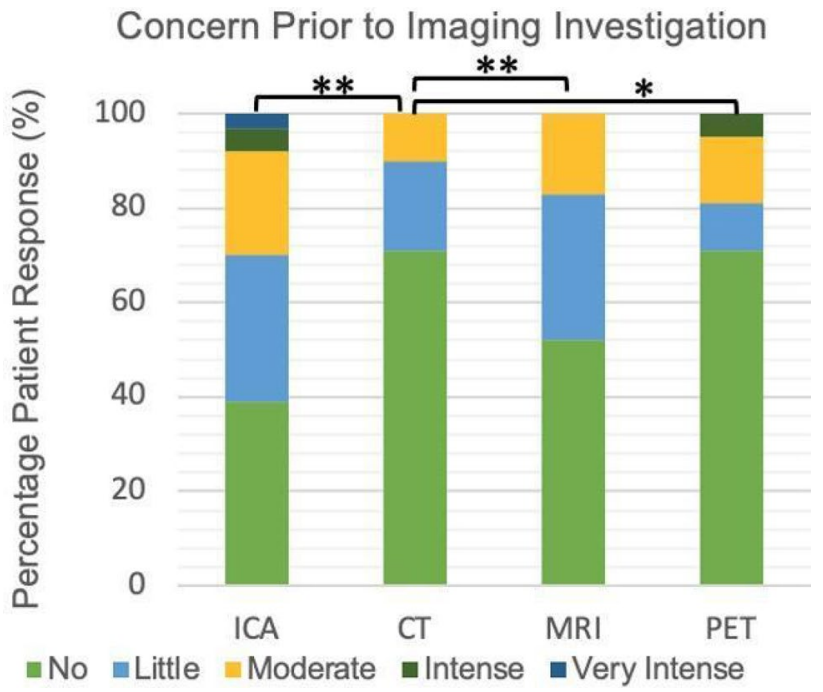

\section{Image 2}

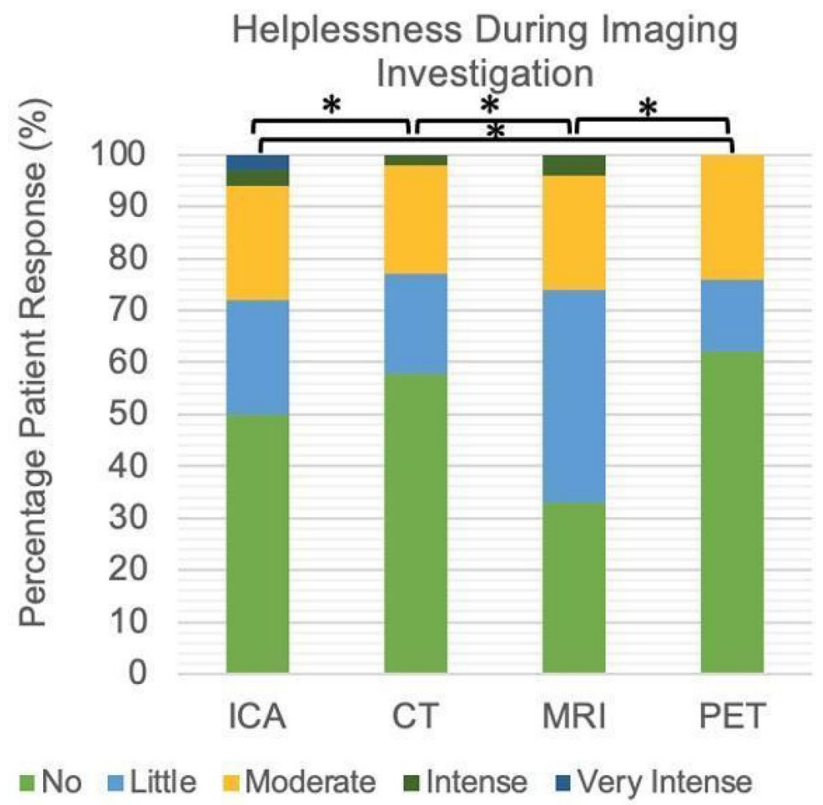

Image 3

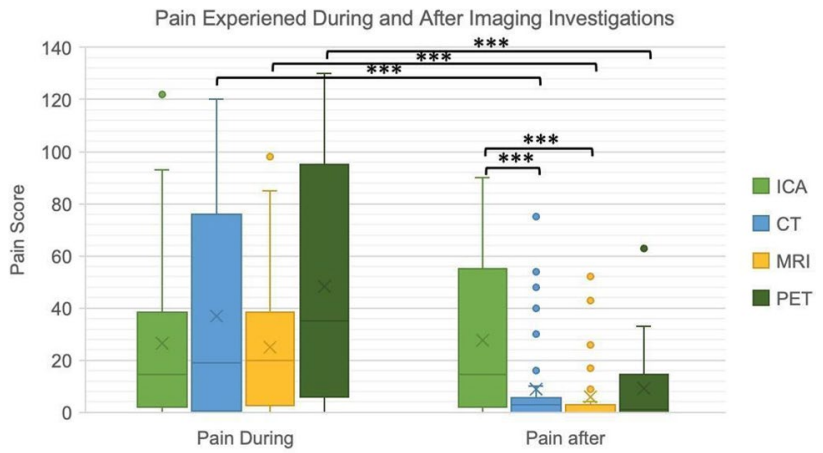




\section{Image 4}

\section{Investigation Preference for Patients Indicating} Specific preference

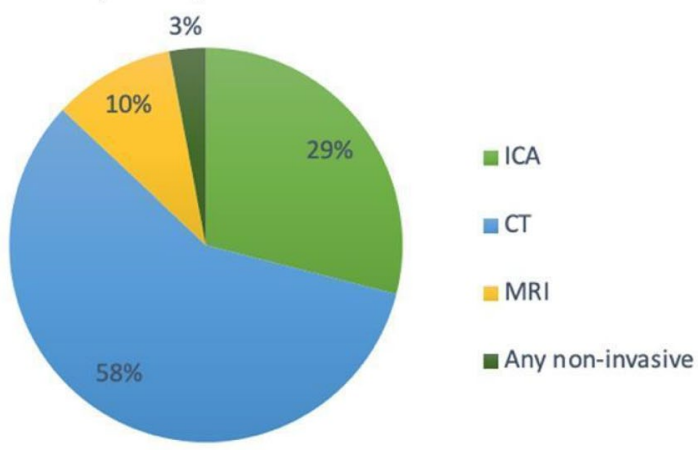

Presenter's first name and last name

Elspeth McLaughlin

Presenter's city and country

Edinburgh, United Kingdom

Presenter's email address

s1604147@ed.ac.uk

\section{8}

\section{Diagnostic Value Of Cardiac Magnetic Resonance Derived Parameters In Patients With Suspected Cardiac Sarcoidosis}

\section{Arpine Kocharyan, Daniel Overhoff, Greta Thater, Uzair Ansari, Julia Michels, Stefan 0. Schönberg, Theano Papavassiliu}

Keywords sarcoidosis, strain analysis, Myocardium, cardiac MRI

\section{Topic Special Focus}

Purpose/Objectives Cardiac sarcoidosis is associated with high mortality. Therefore, early diagnosis and treatment can improve the prognosis of the disease. The goal of this study was to assess the diagnostic value of cardiac magnetic resonance (CMR)-derived parameters in patients with suspected cardiac sarcoidosis, and to compare the results to the standard values obtained from healthy probands.
Methods and Materials CMR examinations of 37 patients with biopsy-proven sarcoidosis were retrospectively evaluated and the results were compared to the normal values obtained from 24 healthy controls. Presence or absence of late gadolinium enhancement (LGE), left ventricular (LV) and right ventricular (RV) function were evaluated. Furthermore, strain analysis was performed by feature tracking.

Results RV ejection fraction (EF) was decreased within the normal range in patients with sarcoidosis $(53 \pm 8.5 \%$ vs $59 \pm 4.6 \%, \mathrm{P}=0.0027)$, while tricuspid annular plane systolic excursion (TAPSE) was normal $(21 \pm 4.5 \mathrm{~mm}$ vs $20 \pm 3.3 \mathrm{~mm}, \mathrm{P}=0.25$ ). Body surface area (BSA)-indexed $\mathrm{RV}$ end-diastolic volume and end-systolic volume (EDV and ESV) were significantly increased $\left(85 \pm 20 \mathrm{ml} / \mathrm{m}^{2}\right.$ vs $68 \pm 13 \mathrm{ml} / \mathrm{m}^{2}, \mathrm{P}=0.0006$ and $40 \pm 14 \mathrm{ml} / \mathrm{m}^{2}$ vs $28 \pm 7 \mathrm{ml} /$ $\left.\mathrm{m}^{2}, \mathrm{P}=0.0002\right)$. Both BSA-indexed LV and RV stoke volume (SV) were increased, too $\left(49 \pm 9 \mathrm{ml} / \mathrm{m}^{2}\right.$ vs $43 \pm 7.6 \mathrm{ml} /$ $\mathrm{m}^{2}, \mathrm{P}=0.008$ and $44.7 \pm 11 \mathrm{ml} / \mathrm{m}^{2}$ vs $39.5 \pm 7.5 \mathrm{ml} / \mathrm{m}^{2}$, $\mathrm{P}=0.049$ ). 2D RV global circumferential strain (GCS) was decreased in patients with sarcoidosis $(-7.96 \pm 3.6 \mathrm{vs}$ $-9.7 \pm 3, \mathrm{P}=0.049)$, while the other RV strain parameters (global radial und longitudinal strain) and all three LV strain parameters were comparable to healthy controls. Only in 7 patients (19\%) LGE was detected.

Conclusion Out of the investigated parameters, RV function and 2D RV GCS were significantly decreased in patients with sarcoidosis. LV function and LV strain parameters were normal. RV function is an important parameter and strain analysis may have additional diagnostic value in detection of cardiac sarcoidosis.

\section{Image 1}

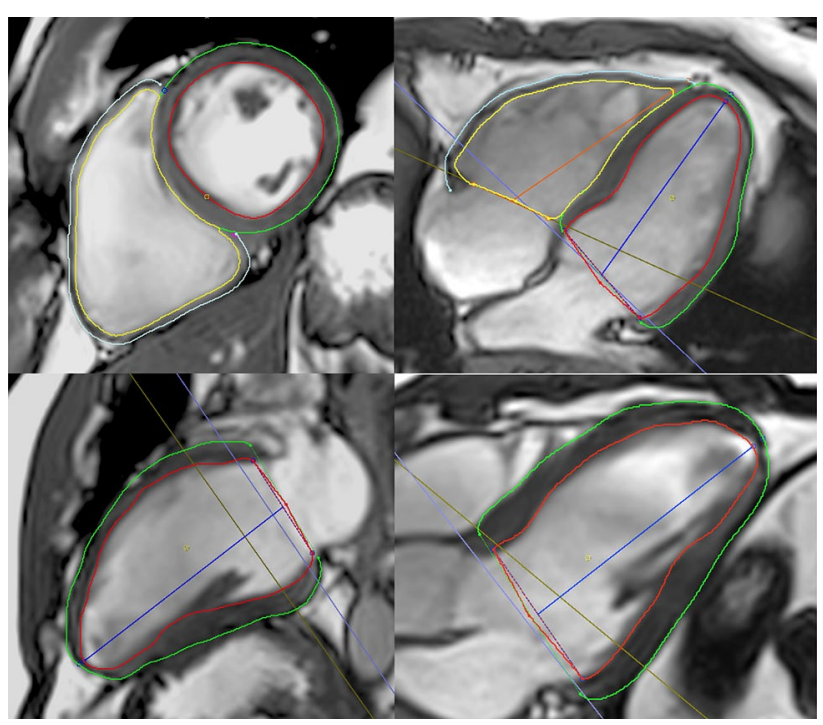




\section{Image 2}

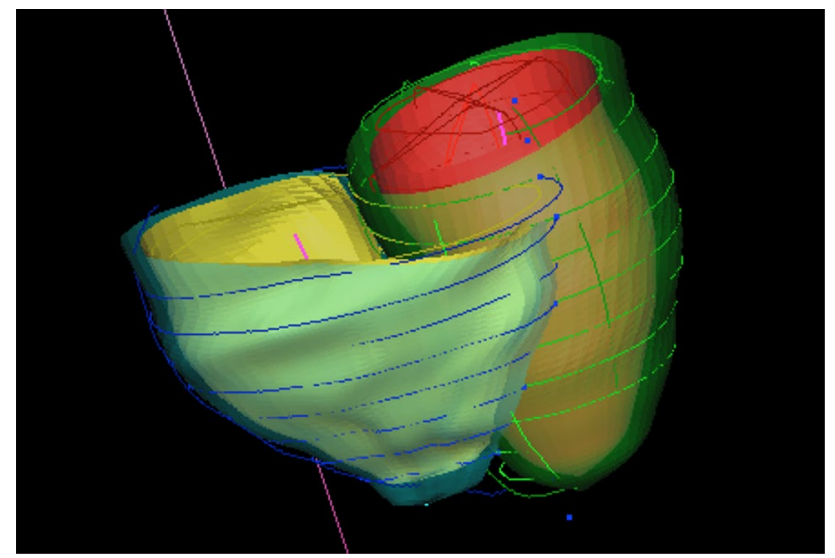

Presenter's first name and last name Arpine Kocharyan

Presenter's city and country

Mannheim, Germany

Presenter's email address

Arpine.Kocharyan@umm.de

\section{9}

\section{CT Density of Epicardial Adipose Tissue in Breast Cancer Patients Treated with Anthracyclines}

\section{Caterina Beatrice Monti, Davide Capra, Moreno Zanardo, Maria Del Mar Galimberti Ortiz, Simone Schiaffino, Francesco Sardanelli, Francesco Secchi}

Keywords Heart, Epicardial Adipose Tissue, Cardiotoxicity, Anthracyclines, Breast Cancer

\section{Topic Area of Interest}

Purpose/Objectives To assess the radiodensity of epicardial (EAT), subcutaneous (SAT) and visceral adipose tissue (VAT) before and after treatment with anthracyclines in a population of breast cancer (BC) patients, and in controls not treated with anthracyclines, to detect a potential role of EAT density as a biomarker of changes related to chemotherapy cardiotoxicity.

Methods and Materials We reviewed BC patients treated with anthracyclines who underwent CT before (CT-0) and after (CT-1) chemotherapy, and age- and sex-matched controls without $\mathrm{BC}$ who did not undergo any cardiotoxic treatment and underwent two CT examinations at comparable intervals. On non-contrast scans, EAT was segmented contouring the pericardium and thresholding between -190 and -30 Hounsfield Units (HU), and SAT and VAT with two regions of interest with a $15-\mathrm{mm}$ diameter thresholded between -195 and $-45 \mathrm{HU}$.

Results 32 female patients along with 32 matched controls were included. There were no differences in age (55 years, interquartile range [IQR] $48-68$ years vs 55 years, IQR $48-67$ years, $p=0.439$ ) and follow-up duration (31 months, IQR 20-53 months vs 27 months, IQR $13-43$ months, $\mathrm{p}=0.162$ ) between patients and controls. Between CT- 0 and CT-1, EAT density decreased in BC patients (from -66 HU, IQR $-71--63 \mathrm{HU}$ to $-71 \mathrm{HU}, \mathrm{IQR}-75--66 \mathrm{HU}, \mathrm{p}=0.003$ ), while it did not vary in controls (from -64 HU, IQR -74--60 $\mathrm{HU}$ to $-67 \mathrm{HU}$, IQR $-71--62 \mathrm{HU}, \mathrm{p}=0.955)$. SAT density increased from CT-0 to CT- 1 in BC patients (from -107 HU, IQR $-111--105$ HU to -105 HU, IQR $-110--100 \mathrm{HU}$, $\mathrm{p}=0.014$ ), whereas it did not change in controls (from -104 HU, IQR -109--101 HU to -106 HU, IQR -109--102 HU, $\mathrm{p}=0.477)$. VAT density did not vary in either BC patients or controls.

Conclusion EAT density appears to be influenced by anthracycline treatment for BC, well-known for its cardiotoxicity, shifting towards lower values indicative of a less active metabolism.

\section{Image 1}

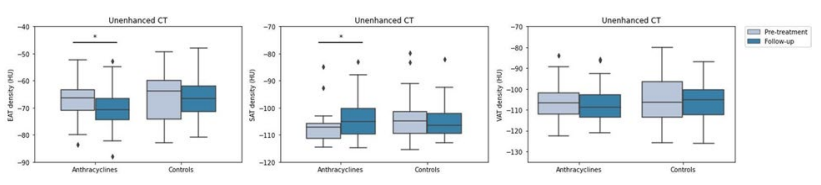

Presenter's first name and last name

Caterina Beatrice Monti

Presenter's city and country

Milano, Italy

Presenter's email address caterinab.monti@gmail.com 


\section{0}

\section{Cardiac MRI of adults with multisystem inflammatory syndrome (MIS-A) after COVID-19}

\author{
Miroslav Muršić, Tihana Balaško Josipović, Maja Hrabak \\ Paar
}

Keywords COVID-19, MRI, Myocarditis

\section{Topic Area of Interest}

Purpose/Objectives Multisystem inflammatory syndrome in adults (MIS-A) is a very rare but severe complication in adults infected with SARS-CoV-2. It's pathophysiology is similar to a parallel multisystem inflammatory syndrome in children-MIS-C which is widely recognized because of dramatic illness in a previously healthy child that can affect the heart, gastrointestinal tract, skin, or brain-very similar to Kawasaki disease $(1,2)$. Although it is difficult to differentiate a severe SARS-CoV-2 case from MIS-A, studies report of a dozen patients who were not on a continuum of severe COVID-19 but developed severe non-pulmonary manifestations without typical viral pneumonia, such as myocarditis (3). Since MIS-A is a rare but potentially lifethreatening complication of previous SARS-CoV-2 infection with scarce available clinical reports, we present our experience with cardiac MRI in two adult patients.

Methods and Materials Out of a few dozen COVID-19 patients who underwent cardiac MRI at our institution, two male patients (Patient 1 26-year-old, Patient 2 40-yearsold) showed matching signs and symptoms and were identified as potential MIS-A patients. The examination was performed on a 1.5 T MRI scanner using our institution's standard protocol for myocarditis that includes steady-state free precession (SSFP) cine imaging, T2-weighted short tau inversion recovery (STIR) imaging, pre-contrast T1 and T2 mapping, post-contrast late gadolinium enhancement (LGE) images after intravenous administration of gadobutrol $(0,15 \mathrm{mmol} / \mathrm{kg})$ and post-contrast $\mathrm{T} 1$ mapping in short- and long-axis views. We compared our results with limited results from available published case reports of this rare and new syndrome. To the best of our knowledge, this is the first report of cardiac MRI in MIS-A patients.

Results Both patients were diagnosed with mild COVID19 few weeks before admission to the hospital with symptoms of fever, chest pain, fatigue, dyspnea and diarrhea. Laboratory findings revealed elevated inflammatory biomarkers, high-sensitivity troponin I and NT-proBNP. Echocardiography showed dilated left ventricle (LV) with severe reduction of LV ejection fraction (EF). Cardiac
MRI in the Patient 1 was performed a week after pulse steroid therapy, intravenous immunoglobulin and heart failure therapy had been started, and showed complete recovery of the LVEF, with slightly increased T1 and T2 relaxation times, and no LGE (Image 1). In the Patient 2 cardiac MRI was performed 2 days after admission to the intensive care unit, and showed dilated LV with LVEF of $41 \%$, severely prolonged $\mathrm{T} 1$ and $\mathrm{T} 2$ relaxation times and slight subepicardial LGE. Follow-up MRI performed after 2 weeks of therapy showed normal LV size, function and T1/T2 relaxation times without any LGE (Image 2).

Conclusion One of life threatening MIS-A manifestations can be post-COVID-19 myocarditis, with rapid improvement of cardiac function after prompt therapy. MRI characteristic findings include prolonged myocardial T1 and T2 relaxation times, myocardial edema, while LGE can be, but is not neccessarily present. Clinicians and radiologists should be familiar with this rare syndrome and MRI is an efficient imaging choice to obtain important information for clinical decision-making.

\section{References}

(1) Hékimian G, Kerneis M, Zeitouni M, et al. Coronavirus disease-19 acute myocarditis and multsystem inflammatory syndrome in adult intensive and cardiac care units. Chest. 2021;159(2):657-662.

(2) Einat Blumfield et al. Imaging Findings in Multisystem Inflammatory Syndrome in Children (MIS-C) Associated With Coronavirus Disease (COVID-19). American Journal of Roentgenology. 2021;216: 507517.

(3) Elie Cogan et al. Multisystem Inflammatory Syndrome With Complete Kawasaki Disease Features Associated With SARS-CoV-2 Infection in a Young Adult. A Case Report Front. Med., 14 July 2020.

Image 1

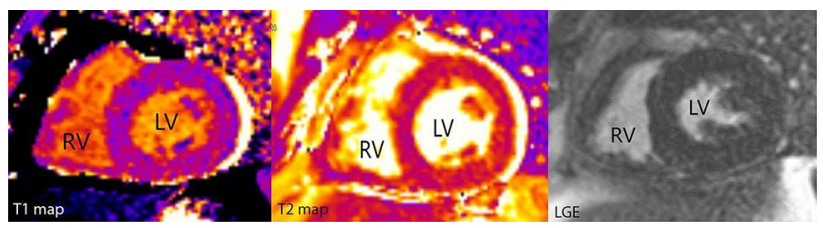




\section{Image 2}

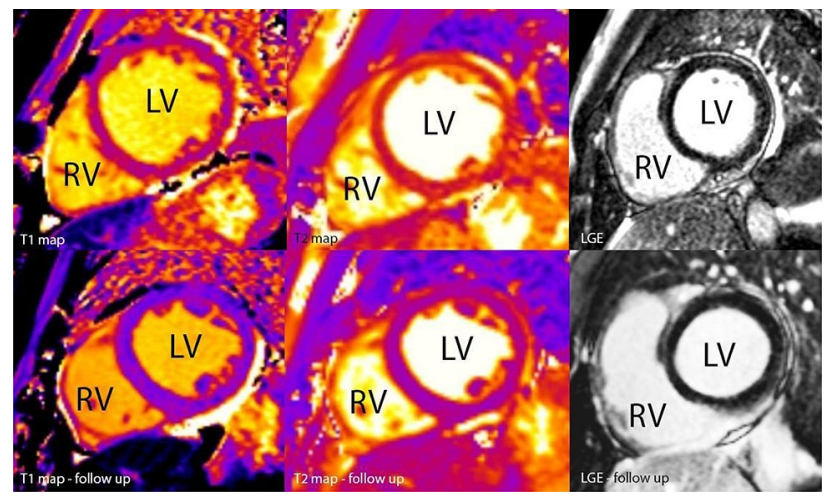

Presenter's first name and last name

Miroslav Muršić

Presenter's city and country

Zagreb, Croatia

Presenter's email address

mursic.miroslav@gmail.com

\section{1}

\section{Myocardial function and characterization in pediatric patients with COVID-19 related Multisystem Inflammatory Syndrome (MISC)}

\section{Davide Capra, Caterina Beatrice Monti, Lucia Mauri, Savina Mannarino, Massimo Garbin, Irene Raso, Francesco Sardanelli, Francesco Secchi}

Keywords Myocardium, Cardiac Magnetic Resonance, Pediatric Patients, COVID-19, COVID-19 related Multisystem Inflammatory Syndrome (MISC)

\section{Topic Area of Interest}

Purpose/Objectives To assess the myocardial status of pediatric patients who had COVID-19-related Multisystem Inflammatory Syndrome (MISC) at cardiac magnetic resonance (CMR).

Methods and Materials We retrospectively reviewed pediatric patients with MISC who underwent echocardiography at hospital admission and CMR at follow-up. A left ventricular (LV) ejection fraction (EF) $<45 \%$ at echocardiography was considered reduced. LV indexed end-diastolic
(EDVi), end-systolic (ESVi) volumes, stroke volume (SV) and EF were calculated from CMR. Moreover, we measured LV native and post-contrast T1 in a short axis (SA) view and in a 4-chamber $(4 \mathrm{CH})$ view, and we assessed $\mathrm{LV}$ global circumferential strain (GCS), global radial strain (GRS), and global longitudinal strain (GLS).

Results 25 patients were included, 6 (24\%) of whom females, with a median age of 11 years $(10-13$ years). Nine $(36 \%)$ patients had reduced EF at echocardiography. The median interval between echocardiography and CMR was 38 days (26-52 days). At CMR LV EDVi was $69 \mathrm{ml} / \mathrm{m} 2(65-74 \mathrm{ml} / \mathrm{m} 2)$; LV ESVi $22 \mathrm{ml} / \mathrm{m} 2(21-25 \mathrm{ml} /$ $\mathrm{m} 2)$; LV SV $62 \mathrm{ml} / \mathrm{m} 2(54-74 \mathrm{ml} / \mathrm{m} 2)$; LV EF $65 \mathrm{ml} /$ $\mathrm{m} 2(62-69 \mathrm{ml} / \mathrm{m} 2)$. No patients had reduced LV EF at CMR. Median GCS was -19.21 (-20.53- -17.05); median GRS was 32.78 (30.84-35.99); median GLS was -11.83 $(-12.69--10.97)$. Native T1 times were $997.38 \mathrm{~ms}$ $(973.78-1040.17 \mathrm{~ms})$ for the SA and $1016.35 \mathrm{~ms}$ (974.11-1068.04 ms) for $4 \mathrm{CH}$, whereas post-contrast $\mathrm{T} 1$ times were $514.28 \mathrm{~ms}(495.42-544.44 \mathrm{~ms})$ and $509.26 \mathrm{~ms}(483.96-535.49 \mathrm{~ms})$ in SA and $4 \mathrm{CH}$ measurements respectively. Patients with reduced EF had increased LV EDVi $(73 \mathrm{ml} / \mathrm{m} 2[71-78 \mathrm{ml} / \mathrm{m} 2]$ vs $68.5 \mathrm{ml} /$ $\mathrm{m} 2[61-73.25 \mathrm{ml} / \mathrm{m} 2], \mathrm{p}=0.015)$ and post-contrast T1 times $(539.82 \mathrm{~ms}$ [499.01-564.48 ms] vs 499.705 [480.11-533.94 ms] for SA, $\mathrm{p}=0.025$; and $535.49 \mathrm{~ms}$ [496.28 - $560.68 \mathrm{~ms}$ ] vs $495.75 \mathrm{~ms}$ [482.99-526.7 ms] for $4 \mathrm{CH}$ measurements, $\mathrm{p}=0.042$ ).

Conclusion Over 35\% of patients with MISC showed a transient reduced EF, that reverted to normal at follow-up. Increased EDVi and post-contrast T1 times at follow-up CMR in such patients may be early signs of myocardial remodeling.

\section{Image 1}
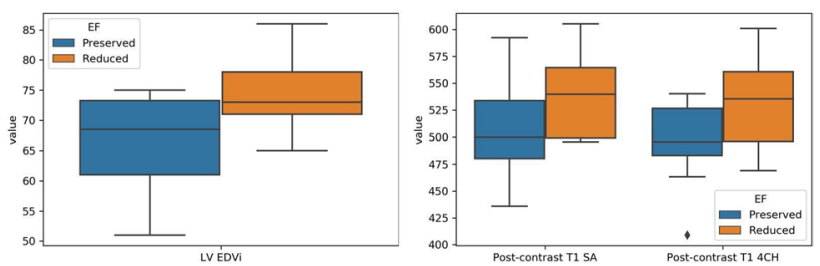

Presenter's first name and last name Davide Capra

Presenter's city and country Milano, Italy

Presenter's email address 
davide.capra@unimi.it

\section{2}

\section{CT Radiomics Of Coronary Artery Calcification Distinguish Vulnerable Plaques In Acute Coronary Syndrome- A Propensity-matched Study With Asymptomatic Subjects With Same Calcified Plaque Burden}

\section{Ming-Ting Wu, Eric Huang}

Keywords Coronary arteries, radiomics, calcification

\section{Topic Area of Interest}

Purpose/Objectives Measurement of coronary artery calcium (CAC) using Agatston score (AS) is widely used to predict the risk of acute coronary syndrome (ACS). However, many ACS victims have AS $<100$, while many asymptomatic subjects have AS $>400$. We hypothesize that $\mathrm{CT}$ radiomics can distinguish the vulnerability of CAC in subjects with similar AS.

Methods and Materials We retrospectively identified 85 patients with ACS who had a CAC scan prior to revascularization. We used 1:2 propensity score matching with traditional risk factors (age, sex, BMI, hypertension, diabetes, dyslipidemia, smoking, family history) and AS ranks to enroll 170 asymptomatic subjects. Radiomics texture features were extracted using the LIFEx package. Generalized linear model and forward conditional logistic regression was used for analysis.

Results For per plaque analysis, lower coefficient of variance, lower median of HU, ?? skewness, and higher kurtosis of Hounsfield (HU) were all favored of ACS ( $p<0.05$ for all). Multivariable analysis showed that higher HU kurtosis $(\mathrm{OR}=18.7, \mathrm{p}<0.001)$ and lower median HU per plaque $(\mathrm{OR}=0.97, \mathrm{p}<0.001)$ are associated with ACS. In subgroup analysis, increased HU kurtosis $(\mathrm{OR}=12.6, \mathrm{p}<0.001)$ and lower median $\mathrm{HU}(\mathrm{OR}=0.97, \mathrm{p}=0.004)$ remain associated with ACS among patients with AS $>100$. Alternatively, among patients with $\mathrm{AS}<=100$, higher HU kurtosis $(\mathrm{OR}=271, \mathrm{p}=0.001)$ and lower $\mathrm{AS}$ per plaque $(\mathrm{OR}=0.86$, $\mathrm{p}=0.023$ ) are associated with increased risk of ACS.

Conclusion Radiomics of CAC plaque suggest that higher HU kurtosis and lower AS or median HU may distinguish the vulnerability of CAC plaques in subjects with the equivalent risk of CAC burden and TRF.

\section{References}

https://doi.org/10.1371/journal.pone.0131254. https://doi.org/10.1001/jama.2013.282535.
Presenter's first name and last name

Ming-Ting Wu

Presenter's city and country

Kaohsiung, Taiwan

Presenter's email address

wu.mingting@gmail.com

\section{3}

\section{Radiological features of caseous calcification of mitral annulus}

\section{Mykhailo Ishchenko, Fedkiv Svitlana, Mariia Tregubova, Yurii Vitkovskyi, Lina Lutsenko, Varvara Parfentyeva}

Keywords Cardiac valves, Caseous calcification, Cardiac $\mathrm{CT}$, Cardiovascular system

Topic Area of Interest

Purpose/Objectives Caseous calcification of mitral annulus (CCMA) is a rare variant of calcification of the mitral valve annulus. Most patients are referred for cardiac CT/ MRI after echocardiography with a preliminary diagnosis of a cardiac tumor. In our clinic, we use MDCT to confirm the diagnosis of caseous calcification of the mitral annulus. The aim of the study was to describe the typical radiological features in patients with caseous calcification of mitral annulus (CCMA).

Methods and Materials Calcification of the mitral valve annulus is a chronic degenerative process that occurs in the elderly, especially in women. Caseous calcification is a less well-known and rarely described pathology that is a relatively uncommon variant of mitral annulus calcification. The prevalence of CCMA in the general population is about $0.068 \%$ and up to $1 \%$ in patients with calcification of the mitral annulus. As a rule, CCMA looks like an ovoid mass consisting of a mixture of calcium, fatty acids, cholesterol located mainly in the posterior parts of the mitral annulus. The pathogenetic mechanism of CCMA has not yet been determined. CCMA is basically a benign process and is diagnosed as a incidental finding. Radiologists who are not familiar with this pathology can interpret it as a cardiac tumor, vegetation, or calcified thrombus - therefore the radiologist needs to be familiar with features of CCMA and careful differential diagnosis is required.

Results CCMA echocardiographically characterized as a large, rounded, mass-like calcification involving the mitral valve annulus, with well-defined margins, an echo-dense periphery, with a central echo-lucent region. 
In most patients, caseous calcification is localized in the posterior parts of the mitral valve annulus, in about $25 \%$ of patients-in other segments of the mitral annulus-in the lateral part or all parts. On MDCT caseous calcification appears as a rounded mass with clear margins with a hypo- or hyperdense center and a calcified peripheral rim. Characteristic features are clear margins, denser calcification at the periphery, and lack of enhancement after contrast administration. Cardiac MRI can be used for differential diagnosis with other pathologies. On MRI, CCMA looks typical and has a hypointense signal on T1, T2, GE sequences. After intravenous administration of gadolinium, most often, no contrast enhancement is observed. Occasionally, peripheral enhancement may appear during delayed post-contrast series.

Conclusion Caseous calcification of the mitral annulus is quite rare in clinical practice, therefore, knowledge of the typical radiological features is very important for the radiologist to be able to differentiate CCMA from a potentially dangerous pathology (for example tumor, vegetation, abscess, thrombus etc.). Echocardiography is the primary imaging technique for CCMA. Multimodal imaging (transesophageal echo, cardiac CT, cardiac MRI) is very useful to confirm the diagnosis of caseous calcification of the mitral annulus and can help avoiding unnecessary manipulation.

\section{References}

Deluca G, Correale M, Ieva R, et al. The incidence and clinical course of caseous calcification of the mitral annulus: a prospective echocardiographic study. J Am Soc Echocardiogr. 2008;21:828-833.

Harpaz D., Auerbach I., Vered Z., Motro M., Tobar A., Rosenblatt S. Caseous calcification of the mitral annulus: a neglected, unrecognized diagnosis. J Am Soc Echocardiogr. 2001;14:825-831.

Blankstein R, Durst R, Picard MH, et al. Progression of mitral annulus calcification to caseous necrosis of the mitral valve: complementary role of multi-modality imaging. Eur Heart J 2009; 30: 304-304.

Fujiwara T, Fujita T, Toda K, et al. Multimodality imaging of caseous calcification of mitral annulus. Eur J Cardiothorac Surg 2012; 41: 451-451.

Egendy IY, Conti CR. Caseous calcification of the mitral annulus: a review. Clin Cardiol. 2013;36:E27-31.

\section{Image 1}
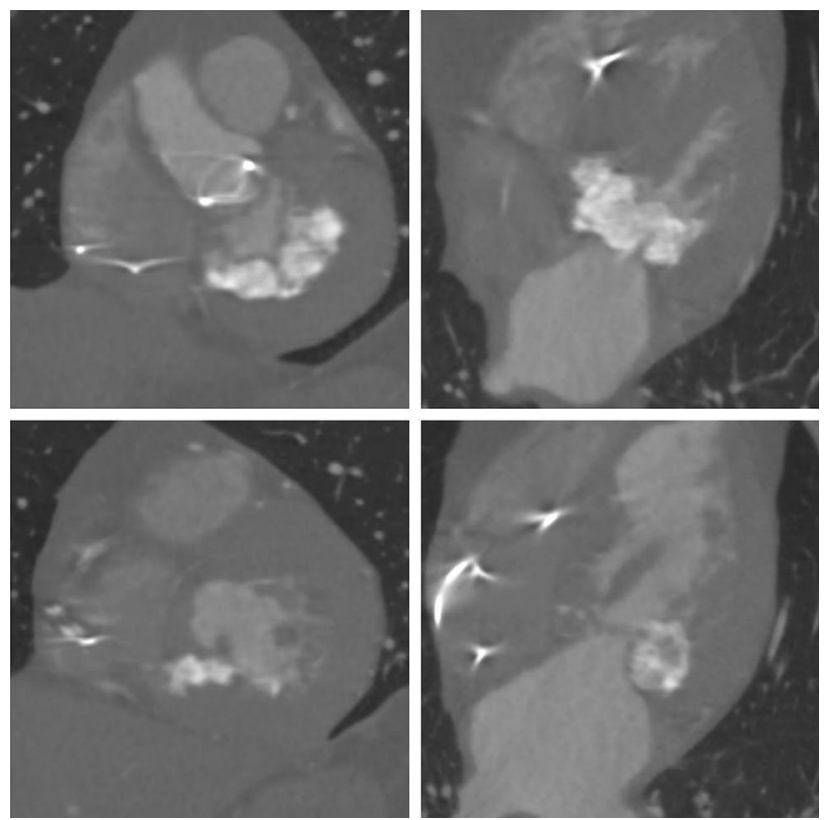

Image 2
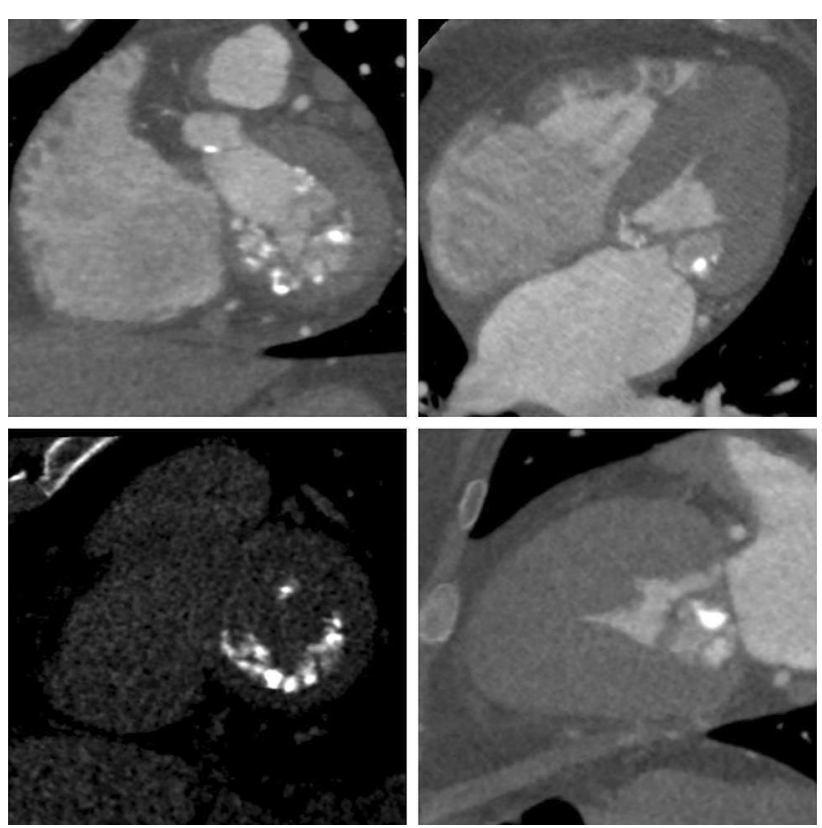


\section{Image 3}
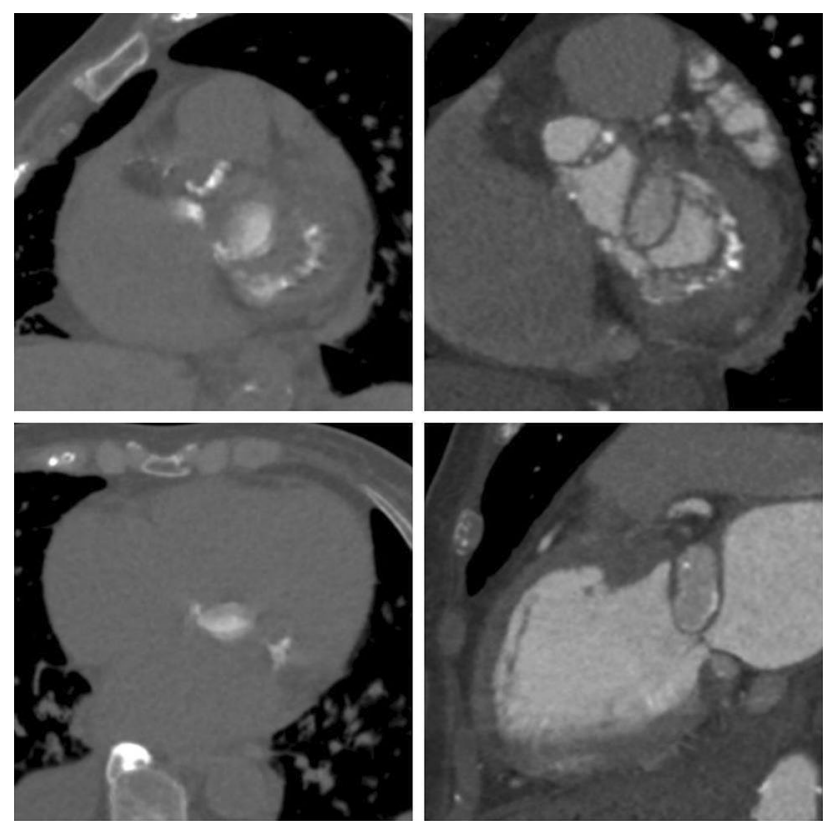

Image 4
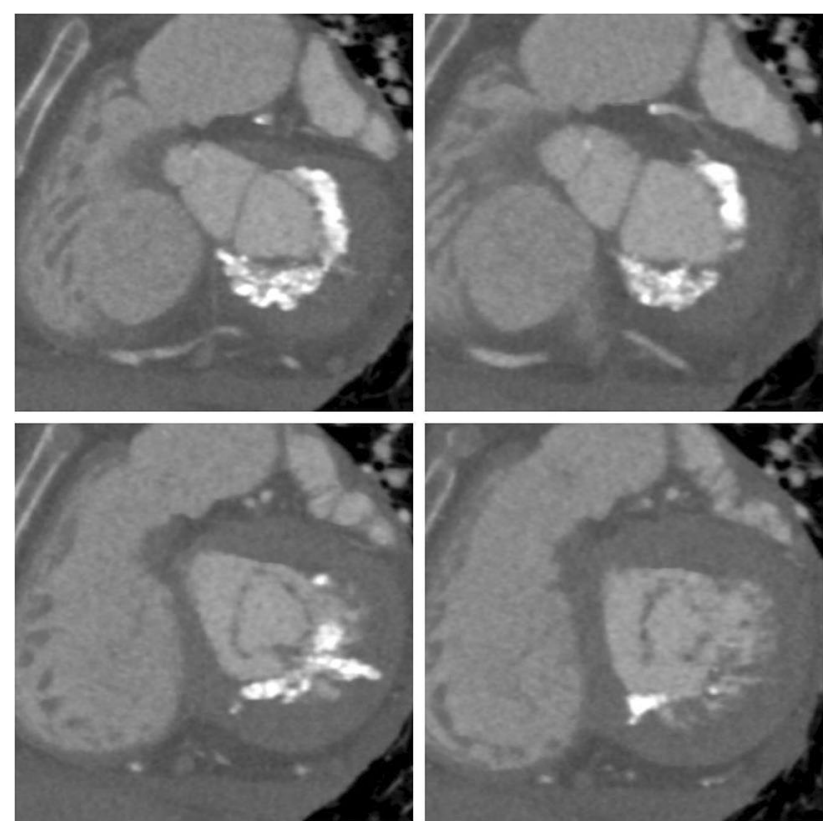

Presenter's first name and last name

Mykhailo Ishchenko

Presenter's city and country

Kyiv, Ukraine

Presenter's email address

holym13@gmail.com

\section{5}

\section{Cardio-MRI of patients with active implanted cardio-systems}

\section{M.A. Shlyappo, Alexandrova Svetlana, Makarenko V.N}

Keywords MRI, artifacts, cardiac MRI, paceakers, implantable cardioverter defibrillator, active implanted heart systems,

\section{Topic Procedure}

Purpose/Objectives The purpose of this study was to evaluate MRI studies conditionally safe for magnetic resonance imaging (MRI) active implanted pacemakers and cardio-version defibrillator (ICD).

Methods and Materials Patients with implanted cardiac devices were examined on the basis of the X-ray diagnostic department,. Three patients with pacemakers and two patients with an implanted cardioverter-defibrillator (electrostimulation systems were of different manufacturers). Four patients had MR-compatible electrodes and one without an ICD electrode. At the time of MRI, all patients were at the stage of dynamic observation and the decision on the state of the myocardium. Before conducting MRI examinations of the heart, all implanted devices were switched by arrhythmologists to MR-compatible mode. MRI of the heart was performed on a $1.5 \mathrm{~T}$ magnetic resonance imager in the supine position of the patient, using a multichannel surface coil for the body and ECG synchronization.

Results The patients were placed under ECG control. In one patient, when placed in a magnetic field, a decrease in heart rate was revealed without signs of artificial "imposition" of a rhythm during the first two minutes-until the period of adaptation of the implanted pacemaker. The study was completed in two patients with implanted 
pacemakers. During the studies, artifacts in the cavities of the right atrium(RA) and right ventricle(RV) from the implanted electrodes were determined, which did not complicate the assessment of images and made it possible to assess the structure of the myocardium and the functional state of the heart. In the study of two other patients with CDI, metal artifacts from the first series (scan plans) were identified, making it difficult to evaluate the images. Artifact reducing sequences were used to reduce artifacts from implanted devices, however, this did not improve image quality and studies were stopped.

Conclusion Today it has become possible to perform MRI examinations of the heart in patients with implanted stimulation systems. However, the device itself can affect the quality of the study, making it impossible to assess the structure of the myocardium. Our examples clearly show the possibility of performing studies subject to the prescribed working conditions, but sometimes, the active implanted device itself, which allows working in a magnetic field, does not allow visualizing the area of interest.

\section{References}

The devices for electrotherapy of the heart, compatible with magnetic resonance tomography. Glushko L.A., Bockeria O.L. Annals of arrhytmology. 2015. Vol. 12. №4. Pp. 225-233.

MRI-compatible pacemaker (first experience of the examination). Shariya M.A., Ternovoy S.K. Russian electronic journal of radiology. 2013. V. 3. № 2. Pp. 92-96.

Revishvili A.Sh., Neminuschiy N.M., Batalov R.E., Gilyarov M.Yu., Golitsyn S.P., Davtyan K.V., Dumpis Ya.Yu., Didenko M.V., Zenin S.A., Ivanitskiy E.A., Komolyatova V.N., Kravtsova L.A., Krivolapov S.N., Kuzovlev A.N., Kuptsov V.V., Lebedev D.S., Lebedeva V K., Linchak R.M., Lomidze N.N., Makarov L.M., Mironov N.Yu., Medvedev M.M., Mikhailov E.N., Nedbaikin A.M., Nesterenko L.Yu.., Romanov A.B., Rzayev F.G., Solokhin Yu.A., Tatarsky R.B., Kharlap M.S., Chapurnykh A.V., Shlevkov N.B., Shubik Yu.V., Cm. Yashin K.E. et co. ALL-RUSSIAN CLINICAL RECOMMENDATIONS FOR CONTROLLING THE RISK OF SUDDEN HEART STOP AND SUDDEN CARDIAC DEATH, PREVENTION AND FIRST AID. Journal of arrhythmology. 2017; (89): 2-104.

\section{Image 1}

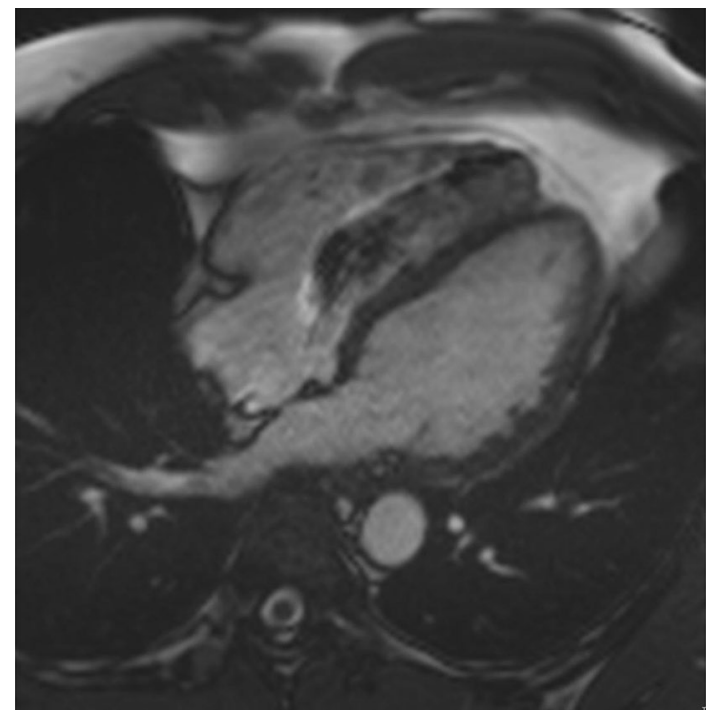

\section{Image 2}

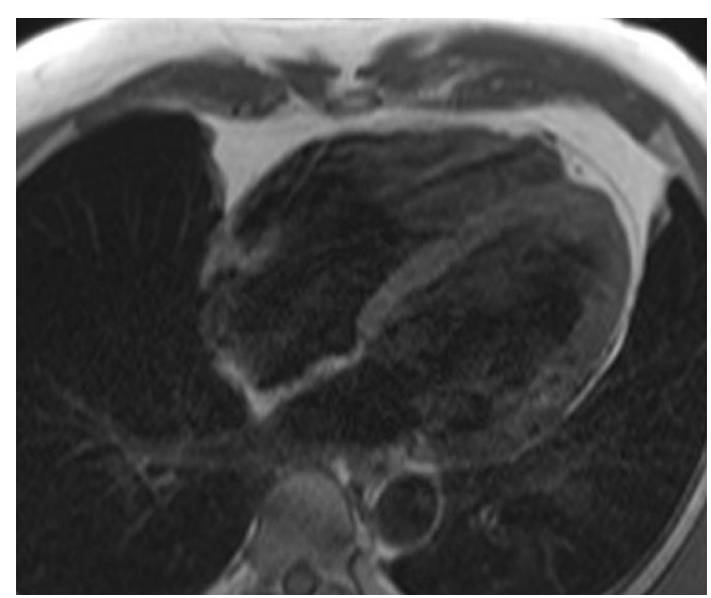




\section{Image 3}

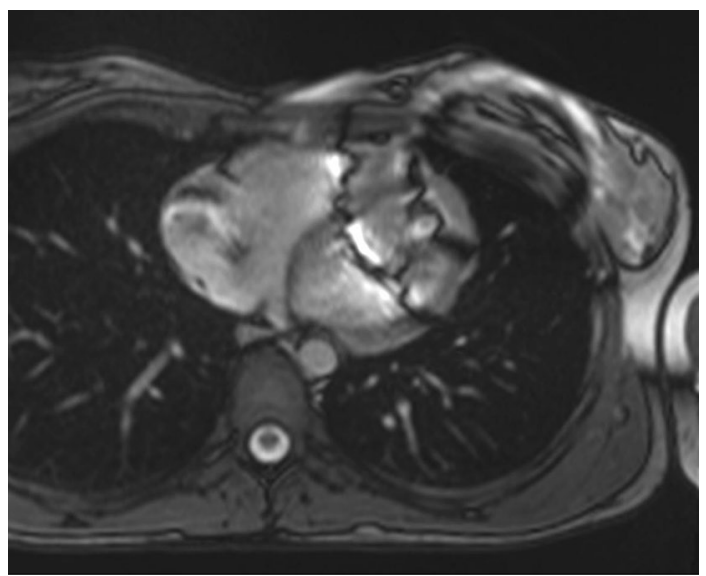

Presenter's first name and last name Shlyappo M.A

Presenter's city and country

Russia, Moscow

Presenter's email address

mariyashlyappo@mail.ru

\section{6}

\section{Cardiac magnetic resonance imaging features as early predictors of left ventricular remodeling in reperfused STEMI patients and impact of high early ASA loading dose as protective for adverse remodeling}

\section{Francesco Cilia, Nicola Galea, Camilla Calvieri, Giacomo Pambianchi, giuseppe mancuso, lacopo Carbone, Marco Francone, Carlo Catalano}

Keywords Myocardium, MR/MR-Angiography, Risk stratification, Ischaemia / Infarction

\section{Topic Special Focus}

Purpose/Objectives Left ventricular (LV) remodelling after ST-elevation myocardial infarction is a complex process, beginning within the first week after STEMI. LV remodelling has been classified as adverse or reverse on the basis of changes in end-diastolic and end-systolic volume at follow-up. Different clinical and Cariac Magnetic Resonance (CMR) parameters had been advocated to predict LV adverse remodeling. Area-at-risk (AAR) measured on T2-weighted images, infarct size quantified as late gadolinium enhanced area, microvascular obstruction and strain analysis are known CMR parameters with prognostic significance, in terms of LV remodeling and major cardiac events. On the other hands, cardioprotective therapies have proved to prevent LV adverse remodeling. Thus, we aimed to evaluate the effect of ASA loading dose on LV remodeling and strain parameters and to identify predictors of adverse remodeling at short term follow up. Methods and Materials Seventy-eight STEMI patients, treated by primary percutaneous coronary intervention (PCI) within $12 \mathrm{~h}$ after symptoms onset between 2011 and 2015, undergoing CMR in the early post-infarction phase (within 5-8 days from symptoms onset) and after 4-6 months, were enrolled retrospectively reviewing our institution's registry. Only patients with paired CMR data on $\mathrm{LV}$ volumes at baseline and at 6-month followup were included in this study. For LV remodeling, on the basis of Bulluck's definition, we divided population into three groups: "adverse" remodeling defined as an increase of end-diastolic LV volume or end-systolic LV volume greater than $12 \%$, "reverse" as a decrease of $12 \%$ of LVESV, and "null" remodeling as other volume percentage increase or decrease less than $12 \%$, between baseline and 4-6 months CMR scans. AAR, infarct size, MVO and strain parameters at baseline have been considered and compared by using ANOVA test.

Results Among 78 STEMI acutely reperfused patients, 38 $(48,7 \%)$ showed adverse remodeling, $22(28,2 \%)$ reverse remodeling and 18 (23\%) no remodeling. At baseline CMR exam, patients with adverse remodeling showed larger AAR, LGE area and MVO extent if compared to other two groups. Regarding CMR strain analysis, only GCS values were worse in adverse remodeling group compared to other two ones. At ROC curve analysis, troponin I, LGE extent baseline, GCS and GLS showed significant sensibility and specificity to detect adverse remodeling at 4-6 months. Then, among 45 STEMI patients, 22 underwent to ASA loading dose (higher than $250 \mathrm{mg}$ orally or i.v.) and 23 to $250 \mathrm{mg}$. Patients receiving higher ASA dose before PCI developed less frequently adverse remodeling, had lower MVO, LGE baseline and LGE at 6 months and better strain parameters if compared to those treated with $250 \mathrm{mg}$ ASA.

Conclusion STEMI patients presenting adverse remodeling reported greater infarct size and worse strain parameters at first CMR exam in the early post-infarction phase if compared to those with reverse or null remodeling. STEMI patients treated with ASA loading dose before PCI showed less adverse remodeling at short term follow up and seems to be protective for $\mathrm{LV}$ adverse remodeling independently 
from nitrates administration, infarct size extent and myocardial damage.

\section{References}

Bulluck H, Carberry J, Carrick D, McEntegart M, Petrie MC, Eteiba H, Hood S, Watkins S, Lindsay M, Mahrous A, Ford I, Oldroyd KG, Berry C. Redefining Adverse and Reverse Left Ventricular Remodeling by Cardiovascular Magnetic Resonance Following ST-SegmentElevation Myocardial Infarction and Their Implications on Long-Term Prognosis. Circ Cardiovasc Imaging. 2020 Jul;13(7):e009937. https://doi.org/10.1161/CIRCIMAGING.119.009937. Epub 2020 Jul 21. PMID: 32,689,822.

\section{Image 1}

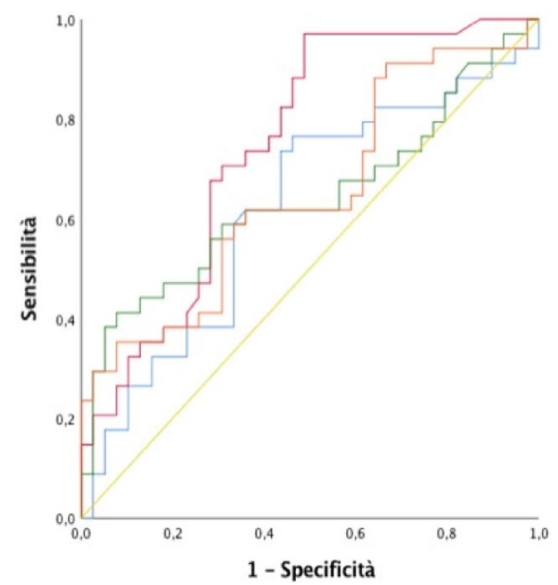

CTnl_peak

- LGE_extent

- GCS

- Riga di riferimento

Presenter's first name and last name

Francesco Cilia

Presenter's city and country

Rome, Italy

Presenter's email address

fcilia91@gmail.com

\section{7}

\section{Role of myocardial T1 and T2 mapping to differentiate acute coronary syndromes}

\section{Giulia Cundari, Valerio Roncaccia, Letizia Ruoli, Giacomo Pambianchi, Laura Vero, lacopo Carbone, Marco Francone, Carlo Catalano, Nicola Galea}

Keywords Cardiac Magnetic Resonance;, Mapping;, Acute coronary syndrome

Topic Imaging Technique

Purpose/Objectives To investigate whether myocardial native T1 (nT1) and T2 values may help in the differential diagnosis of the three main different etiologies of acute coronary syndrome: myocarditis (AM), myocardial infarction (AMI) and Tako-Tsubo syndrome (TTS).

Methods and Materials We retrospectively selected 134 patients admitted for acute coronary syndrome who underwent cardiac magnetic resonance (CMR) between 2015 and 2021. All those patients performed a complete CMR protocol: 63/134(47\%) were AM, 54/134(40\%) AMI and $17 / 134(13 \%)$ TTS, according to the conventional diagnostic patterns using T2-weighted and late enhanced images. 30 healthy subject were also recruited as control group. CMR protocol included non-contrast MOLLI 5(3)3 and T2-3pt-GRE, for nT1 and T2 mapping respectively. Left ventricular myocardial $\mathrm{nT} 1$ and $\mathrm{T} 2$ values have been measured on a 16-segments-based approach. Thresholds of normal nT1 and T2 myocardial values have been established using the 95 upper percentile of control group. In each patient, the highest segmental values (Max-) and the average values among all those segments with increased nT1/ T2 (Ave-) have been assessed in both $\mathrm{nT} 1$ and T2 maps. Statistical analysis was performed using ANOVA, student $\mathrm{t}$-test and ROC curve analysis.

Results Max-T1, Ave-T1, Max-T2 and Ave-T2 have been assessed in all AM (1144.6 $\pm 76.7 \mathrm{~ms}$, $1096.3 \pm 42.1 \mathrm{~ms}, 58.1 \pm 6.18 \mathrm{~ms}$ and $53.7 \pm 2.75 \mathrm{~ms})$, AMI $(1248.3 \pm 94.7 \mathrm{~ms}, 1129.9 \pm 44.9 \mathrm{~ms}, 63 \pm 7.5 \mathrm{~ms}$ and $55.9 \pm 3.2 \mathrm{~ms})$ and TTS $(1242.3 \pm 48.4 \mathrm{~ms}$, $1127.9 \pm 21.6 \mathrm{~ms}, 63.9 \pm 5.1 \mathrm{~ms}$ and $56.8 \pm 2.94 \mathrm{~ms}$ ) patients. All values were significantly higher in patients with AMI/TTS as compared to AM and controls [p $<0.001$ for all comparisons]. No significant differences have been found in the comparison between AMI and TTS values. ROC analysis showed good performance in distinguishing 
AMI/TTS Vs AM/controls (AUC:0.77-0.87), whereas in the comparison between AM and controls was excellent for T1 values (AUC:0.966-0.981), good for Max-T2 (AUC:0.832) and sufficient for Ave-T2 (AUC:0.669).

Conclusion Max- and Ave- T1/T2 values vary between different forms of acute myocardial injury and improving CMR diagnostic performance in the acute scenarios, and appear promising to obtain robust and accurate diagnosis also without the use of contrast agent.

Presenter's first name and last name Giulia Cundari

Presenter's city and country

Rome, Italy

Presenter's email address giulia.cundari@uniroma1.it

\section{1}

\section{Coronary Computed Tomography Angiography in acute chest pain: a sustainable model with remote support}

\section{Livia Marchitelli, Federica Catapano, Andrea Fiorelli, Pietro Sedati, Marco Francone, Carlo Catalano, Nicola Galea}

Keywords CT/CT-Angiography, Coronary artery disease, emergency department

Topic Imaging Technique

Purpose/Objectives To propose a sustainable model of coronary computed tomography angiography (CCTA) use in acute coronary syndrome (ACS) in an emergency department (ED) facility using a partially based teleradiology reporting model. We also analyzed impact of the protocol on patient's outcome over a 5-year follow-up by focusing on the occurrence of major adverse cardiovascular events (MACEs).

Methods and Materials From an initial cohort of 637 subjects admitted to the ED with chest pain during a 12-month period, 104 consecutive patients with low to intermediate risk of ACS according to the HEART score system were selected. All patients underwent CCTA within $6.2 \pm 3.2 \mathrm{~h}$ from admission. Medical reporting was based on a model combining on-site physician and a remote radiologist supported by a web client-based teleradiology system, covering a 24/7 service with rotation of 5 dedicated cardiac-CT specialists. CCTA findings were correlated with the incidence of MACEs over a 5-year follow-up.
Results CCTA ruled-out significant CAD (CAD-RADS: $0-2)$ in 76 patients $(73.1 \%), 65$ showing absent or minimal luminal stenosis and 11 with mild degree lesions. While 28 subjects (CAD-RADS: 3-5) showed respectively moderate $(7.7 \%)$ to severe $(19.2 \%)$ coronary artery disease and were directly referred to functional tests or invasive angiographic examination. The mean discharge time in patients who performed CCTA was $10.8 \pm 5.9 \mathrm{~h}$, while patients addressed to standard evaluation at ED had a mean discharge time of $24.8 \pm 12.6 \mathrm{~h}(\mathrm{p}<0.05)$. Regarding the reporting model, $76 / 104$ cases $(73 \%)$ were reported using a teleradiology platform, with slightly shorter reporting times, without significant impact on patient's discharge $(10.4 \pm 5.3$ vs $12.2 \pm 6.7 \mathrm{~h}$, p: 0.34$)$. MACEs have been higher at 5-year follow-up for the group with CAD-RADS $>$ or $=3$ compared to the group with CADRADS $<3$ (n: 6/66 Vs. 8/28, respectively, p <0.05).

Conclusion CCTA assessment of patients with ACP enables to quickly rule-out ACS, avoiding inappropriate hospitalization and waste of resources, and to identify among symptomatic patients those with higher risk of MACEs at 5-year follow-up. A partial teleradiology based 24/7 CCTA service allowed a rapid discharge of patients with suspected ACS from ED.

Presenter's first name and last name

Livia Marchitelli

Presenter's city and country

Rome, Italy

Presenter's email address

livia.marchitelli@uniroma1.it

\section{2}

\section{Automatic Aorta Segmentation in PET/CT Using a 3D U-Net}

Gijs van Praagh, Pieter Nienhuis, Melanie Reijrink, Bruce Spottiswoode, Fei Gao, Niek Prakken, Kim Mouridsen, Ronald Borra, Riemer Slart

Keywords PET/CT, Aorta, Artificial intelligence, Segmentation

Topic Procedure

Purpose/Objectives Non-invasive imaging techniques play a central role in identification, stratification, and follow-up of infectious and inflammatory vascular disease and could 
be of major importance for preventing and delaying vascular damage and its complications in high-risk patients. Manual or semi-automatic quantification of these diseases is timeconsuming. Besides that, most fully automatic analysis software fails to integrate both the anatomical and the functional information obtained from hybrid nuclear medicine images like positron emission tomography (PET) with computed tomography (CT). The aim of this study is to develop an accurate, fast, and fully automatic algorithm to segment the aorta on low-dose CT (LDCT) images as a navigator for PET-assessed vascular disease.

Methods and Materials Eighteen non-contrast LDCT scans from [18fluor]fluordeoxyglucose ([18F]FDG) PET/CT scans were used. For all LDCT scans, the aorta from aortic valve to iliac bifurcation was manually delineated and used as 'ground truth'. Split, rotation, and noise addition augmentation techniques were used on fifteen scans to create 100 3D patches, which were split into a training $(n=80)$ and validation $(n=20)$ set. Three scans without augmentation were used as test set. Cropping, normalization, and zerocentering were used for image preprocessing to speed up and improve accuracy of the training. No down sampling was used. A U-Net architecture was modified to process 3D inputs and outputs. The Dice Similarity Metric (DSM) was used to assess algorithm performance.

Results The test set yielded a mean DSM of $0.846 \pm 0.020$ for the entire aorta. The run time for both test sets with the trained 3D U-Net was on average $2 \mathrm{~s}$ per patient, whereas the manual delineation was in the order of 30 min per patient.

Conclusion A modified 3D U-Net was trained for segmentation of the aorta on non-contrast LDCT obtained from PET/ CT scans. Comparisons of ground truth and segmented aorta from automatic segmentations using the trained 3D U-Net demonstrated excellent concordance. This method can be used for faster and more in-depth analyses of PET tracer uptake in the vascular wall and potentially applied for fast and accurate quantification of vascular inflammatory and infectious diseases.

\section{Image 1}

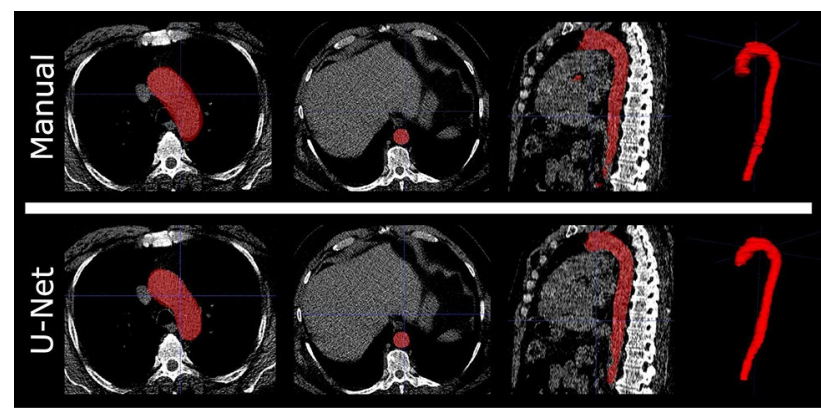

Presenter's first name and last name

Gijs van Praagh

Presenter's city and country

Groningen, the Netherlands

Presenter's email address

g.d.van.praagh@umcg.nl

\section{3}

\section{Assessment of ascending and descending aorta in pediatric patients after surgical treatment of Tetralogy of Fallot using 4D Flow MRI imaging}

\section{Agata Wisz, Zbigniew Olczak, Magdalena Machnikowska- Sokołowska, Katarzyna Gruszczyńska}

Keywords 4D Flow, Cardiac MRI, Pediatric Radiology, Tetralogy of Fallot

\section{Topic Imaging Technique}

Purpose/Objectives Tetralogy of Fallot is the most common cyanotic congenital heart disease. Surgical repair at early age significantly improves long term- survival of these patients. 4D Flow sequence is a new tool for visualization and quantification of blood flow in heart and large vessels. It can also provide information about physiological parameters such as wall shear stress (WSS) and loss of kinetic energy and how are they altered in patients after surgical correction of their congenial heart disease. Since use of this imaging technique and studies including evaluation of aorta parameters in pediatric patients are limited to date, the aim of this study is to analyze maximum wall shear stress (WSS) in ascending and descending aorta in a group of patients who underwent surgical correction of Tetralogy of Fallot.

Methods and Materials We retrospectively analyzed 14 patients ( 7 months- 16 years old) with history of surgical treatment of Tetralogy of Fallot, who underwent regular 4D flow imaging. All imaging was performed on 1,5 $\mathrm{T}$ Artist GE MRI scanner (GE Healthcare, Milwaukee, WI). Post-processing was executed with CVI 42 (Circle Cardiovascular Imaging). We used segmentation tool of software to track centerline of the vessel (aorta), after which we manually determined the points of maximum wall shear stress (WSS) measurement on both ascending and descending aorta in each patient. 6 patients (8-17 years old) with no congenital heart disease and normal heart 
function with 4D flow examination from our database served as control group.

Results There was no significant difference in maximum WSS $[\mathrm{Pa}]$ in ascending aorta between study and control group. In study group mean maximum WSS in ascending aorta was $0,378 \mathrm{~Pa}[0,183-0,574 \mathrm{~Pa}]$, whereas in control group $0,341 \mathrm{~Pa}[0,212-0,47 \mathrm{~Pa}]$. We observed slightly elevated maximum WSS $[\mathrm{Pa}]$ in descending aorta in our study group, compared to control. In study group mean maximum WSS in descending aorta was $1,257 \mathrm{~Pa}[0,651-2,058 \mathrm{~Pa}]$ and in control group $0,670 \mathrm{~Pa}[0,381-0,941 \mathrm{~Pa}]$.

Conclusion Patients after surgical treatment of Tetralogy of Fallot may be prone to elevated wall shear stress in descending aorta, which could potentially lead to weakening of vessel wall, therefore close monitoring towards complications such as aortic aneurysm in years following surgery should be applied.

Presenter's first name and last name Agata Wisz

Presenter's city and country

Katowice, Poland

Presenter's email address agwisz@gmail.com

\section{6}

\section{Positioning of the Image Plane in Phase Contrast CMR Impacts Aortic Stenosis Assessment - Where to Lay the Right Layer}

\section{Felix Troger, Martin Reindl, Christina Tiller, Magdalena Holzknecht, Mathias Pamminger, Ivan Lechner, Christian Kremser, Sebastian Reinstadler, Johann Willeit, Axel Bauer, Bernhard Metzler, Gert Klug, Agnes Mayr}

Keywords aortic valve, phase-contrast $\mathrm{cmr}$, aortic stenosis

\section{Topic Area of Interest}

Purpose/Objectives Phase-contrast cardiovascular magnetic resonance imaging (PC-CMR) has become an emerging tool in characterizing aortic stenosis (AS). While it is increasingly used in quantifying stenosis degree, it still remains unclear at what distance from valve level (VL) this method yields the most reliable results. The aim of this study was to determine the PC-CMR imaging level above the stenotic aortic valve, which generates the most valid measures of flow velocity and volume compared to cardiac catheterization.
Methods and Materials 51 patients with moderate to severe AS underwent cardiac catheterization and CMR including cine-imaging and PC-CMR. A total of 243 imaging planes parallel to the aortic valve were measured via PC-CMR, ranging from $22 \mathrm{~mm}$ below to $24 \mathrm{~mm}$ above VL at enddiastole. Aortic valve area (AVA) via PC-CMR was calculated as mean flow volume divided by peak velocity over the whole systole. In all layers, stroke volume (SV) and AVA were determined and compared to volumetric SV and invasive AVA via the Gorlin-formula, respectively.

Results Above VL, SV by PC-CMR compared to cinevolumetry showed no significant differences depending on image plane position and correlated strongly with volumetric measurements ( $\mathrm{r}: 0.647, \mathrm{p}<0.001$, bias: $1 \mathrm{ml}$, $\mathrm{p}=0.540$ ). AVA assessment in layers from $0-10 \mathrm{~mm}$ above VL differed significantly between PC-CMR and invasive measurement (bias: $0.13 \mathrm{~cm}^{2}, \mathrm{p}<0.001$ ). In contrast, AVA values by PC-CMR measured 10-20 mm above VL showed moderate agreement with invasive determination $(\mathrm{r}$ : $0.444, \mathrm{p}=0.005)$ without any significant bias $\left(0.001 \mathrm{~cm}^{2}\right.$, $\mathrm{p}=0.974)$. Within these measurements, the layer at $15 \mathrm{~mm}$ above VL displayed the lowest bias $\left(-0.05 \mathrm{~cm}^{2}, \mathrm{p}=0.516\right)$. Conclusion PC-CMR measurements at $0-10 \mathrm{~mm}$ above the stenotic aortic valve should be avoided due to significant AVA overestimation compared to invasive determination. AVA assessment by PC-CMR between 10 and $20 \mathrm{~mm}$ above VL does not differ from invasive measurements, with the lowest intermethodical bias measured $15 \mathrm{~mm}$ above the valve.

Image 1

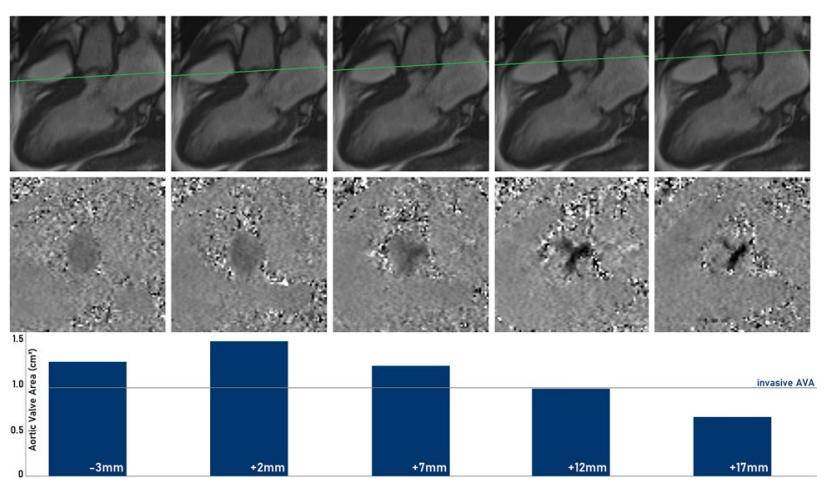

Image 2 
Felix Troger
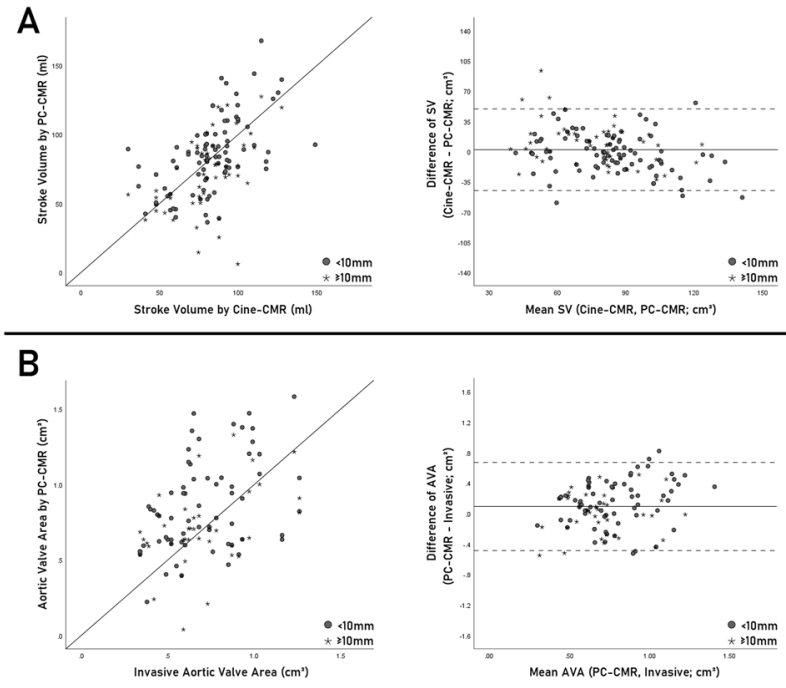

Image 3

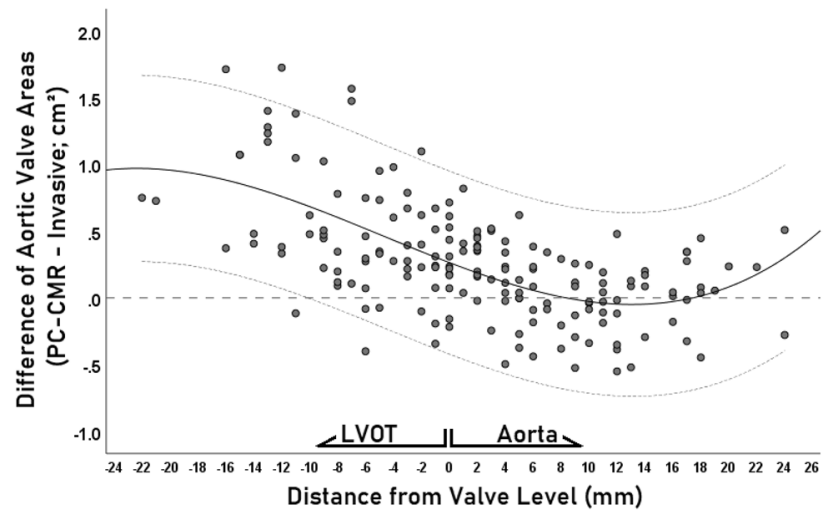

Image 4

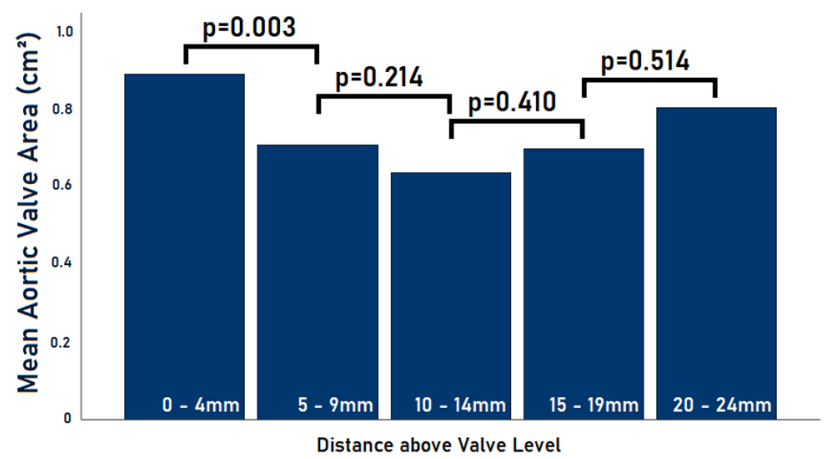

Presenter's first name and last name
Presenter's city and country

Innsbruck, Tyrol, Austria

Presenter's email address

felix.troger@i-med.ac.at

\section{7}

\section{Compressed sensing acceleration of cardiac cine imaging allows reliable and reproducible assessment of strain values of the left atrium}

\section{Sebastian Altmann, Moritz Halfmann, Joe Schoepf, christoph.dueber@unimedizin-mainz.de, Karl-Friedrich Kreitner, Akos Varga-Szemes, Tilman Emrich}

Keywords MR, Cardiac-Imaging, Compressed Sensing, Atrial deformation analysis, Strain, atrial imaging

Topic Imaging Technique

Purpose/Objectives To compare atrial strain parameter derived from highly accelerated compressed sensing (CS)based cine sequences in comparison to conventional (Conv) cine imaging.

Methods and Materials CS and Conv cine sequences were acquired in 46 subjects [ 30 healthy volunteers (HV) and 16 patients with heart failure with reduced ejection fraction (HFrEF)] using a 3 T MR scanner in this single-center study. Deformation analysis of the left (LA) atrium was performed in both sequences to evaluate long axis and AV junction strain. Inter-sequence agreement was analyzed using correlation, intraclass-correlation (ICC) and Bland-Altman analysis.

Results CS-based cine imaging lead to a $69 \%$ reduction of acquisition time. There was significant difference in atrial strain parameters between CS and Conv cine, e.g. LA Long axis strain [Conv $30.3 \% \pm 15.5$, CS $27.4 \% \pm 13.1, \mathrm{P}=0.003$ ] or AV junction strain [Conv $26.6 \% \pm 13.4$, CS $24.6 \% \pm 11.9$, $\mathrm{P}=0.016]$. However, there was high correlation between the techniques, yielding excellent ICC $(>0.93)$ and correlation $(r>0.95)$ (Fig. $1 \&$ 2). CS-based parameters [Long axis strain $\mathrm{AUC}=0.956$, AV junction strain $\mathrm{AUC}=0.948$ ] showed equivalent diagnostic ability compared to Conv cine imaging [Long axis strain AUC $=0.969$, AV junction strain AUC $=0.0 .948]$ to differentiate between HV and HFrEF (Fig. 3).

Conclusion Atrial deformation analysis using CS cine imaging is feasible with relevant reduction of acquisition time, 
therefore strengthening the role of CS in clinical CMR for atrial imaging.

\section{Image 1}

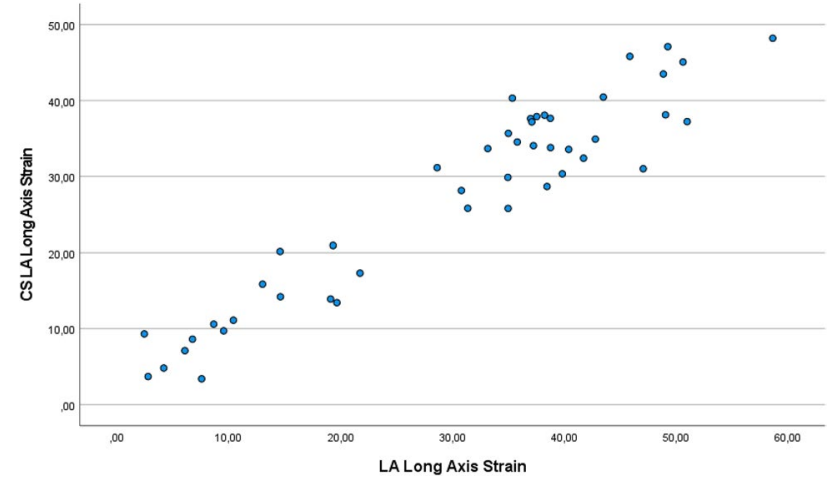

Image 2

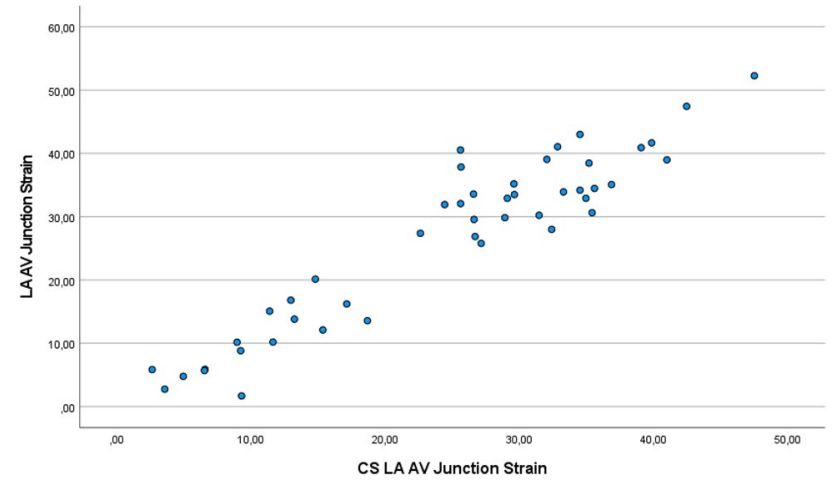

Image 3

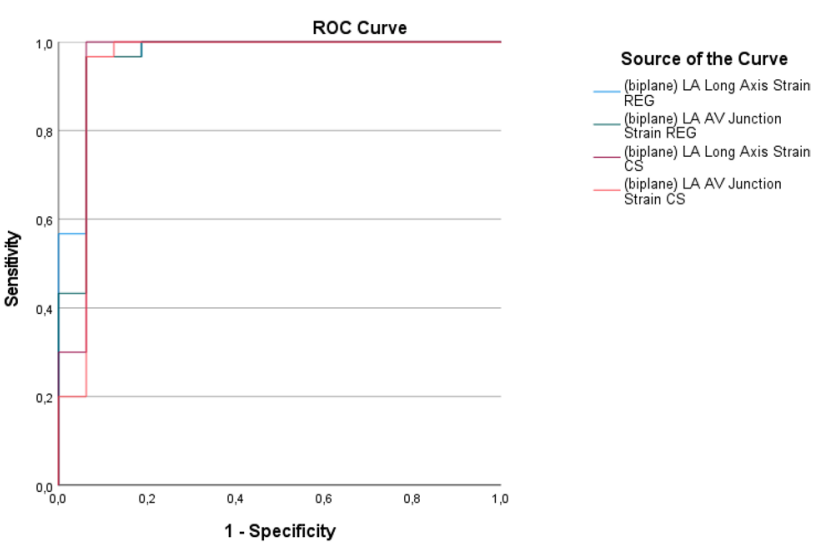

Presenter's first name and last name

Sebastian Altmann

Presenter's city and country

Mainz, Germany

Presenter's email address

sebastian.altmann@unimedizin-mainz.de

\section{8}

Arterial thrombosis associated with COVID-19 infection evaluated by computed tomography angiography

Mariia Tregubova, Fedkiv Svitlana, Kostyantyn Rudenko, Yurii Vitkovskyi, Mykhailo Ishchenko, Varvara Parfentyeva, Lidiya Nevmerzhitskaya, Polina Danchenko

Keywords Aorta, Peripheral arteries, CT/CT-Angiography, Ischaemia / Infarction, Embolism / Thrombosis

Topic Special Focus

Purpose/Objectives During the peak of the coronavirus disease 2019 (COVID-19) pandemic, venous thromboembolism became well-recognised as a common complication of severe COVID-19 disease. Arterial thrombosis has been less well recognised, although it is increasingly reported, mainly in the context of lower limb ishchemia and cerebrovascular stroke.Even though arterial clots are a rare entity, the procoagulant nature of COVID-19 is associated with thrombosis at atypical sites and should be considered in patients with severe coagulation abnormalities. The goal of this study was to demonstrate and evaluate the main 
features of arterial thrombosis of COVID-19-positive patients on computed tomography angiography (CTA).

Methods and Materials We studied 7 cases of severe arterial thrombotic events in patients with confirmed COVID19 infection, admitted at Amosov National Institute of Cardiovascular Surgery from October 2020 to February 2021. COVID-19 diagnosis was made by polymerase chain reaction assay and chest computed tomography (CT) associated to respiratory symptoms in all cases. Arterial thrombosis was diagnosed clinically and confirmed by a color Doppler duplex ultrasonography and CTA. All studies were performed on 640-slices CT scanner (Toshiba Aquilion One, Japan).

Results In most cases, the degree of pulmonary involvement reflects the severity of the course of COVID-19 infection. However, vascular thrombosis can have a significant impact on the outcome of the disease. All the examined patients in addition to severe COVID-19 infection had a history of cardiovascular disease. The first case of a patient with an obstructive thrombosis of the abdominal aorta (Fig. 1A) and bilateral common iliac artery thrombosis. Also the CT study showed filling defects in the superior vena cava and right atrium (Fig. 1B). It the second and third cases there was thrombosis of the right femoral artery ( Fig. 2). In the fourth case patient had left popliteal artery thrombosis. The fifth patient had occlusion of a distal segment of the superior mesenteric artery (Fig. 3). The sixth case is of a patient who had renal and spleen infarcts. In the seventh case patient had left middle cerebral artery stroke (Fig. 4).

Conclusion COVID-19 could predispose to both venous and arterial thromboembolism, in an exaggerated immune response to the virus, especially in severe patients. It has also been proposed that coagulopathy is characterized by more dire prognosis in COVID-19 patients. Knowledge of the possibility of thromboembolic complications can increase their early detection in this group of patients. CT is crucial for assessment not only of COVID-19 pneumonia, CTA has a key role concerning diagnosis and management in patients affected by COVID-19 - associated arterial thrombosis, such as aortic thrombosis, myocardial infarction, mesenteric ischemia, cerebrovascular stroke and limb ischemia.

\section{References}

Cui S, Chen S, Li X, Liu S, Wang F. Prevalence of venous thromboembolism in patients with severe novel coronavirus pneumonia. J Thromb Haemost. 2020.

Madjid M, Safavi-Naeini P, Solomon SD, Vardeny O. Potential effects of coronaviruses on the cardiovascular system: a review. JAMA Cardiol. 2020.
Xiong TY, Redwood S, Prendergast B, Chen M. Coronaviruses and the cardiovascular system: acute and long-term implications. Eur Heart J. 2020;41(19):1798-1800.

Klok FA, Kruip MJHA, van der Meer NJM, et al. Incidence of thrombotic complications in critically ill ICU patients with COVID-19. Thromb Res. 2020;

Zhang Y, Cao W, Xiao M et al. Clinical and coagulation characteristics of 7 patients with critical COVID-2019 pneumonia and acro-ischemia. Chinese Journal of Hematology 2020; 41.

Varga Z, Flammer A, Steiger P, et al. Endothelial cell infection and endotheliitis in covid-19. Lancet 2020;395:1417-8.

\section{Image 1}

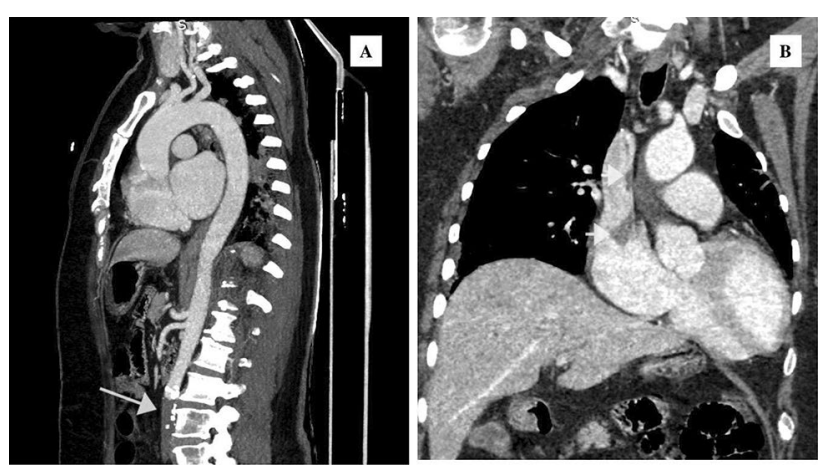

Image 2
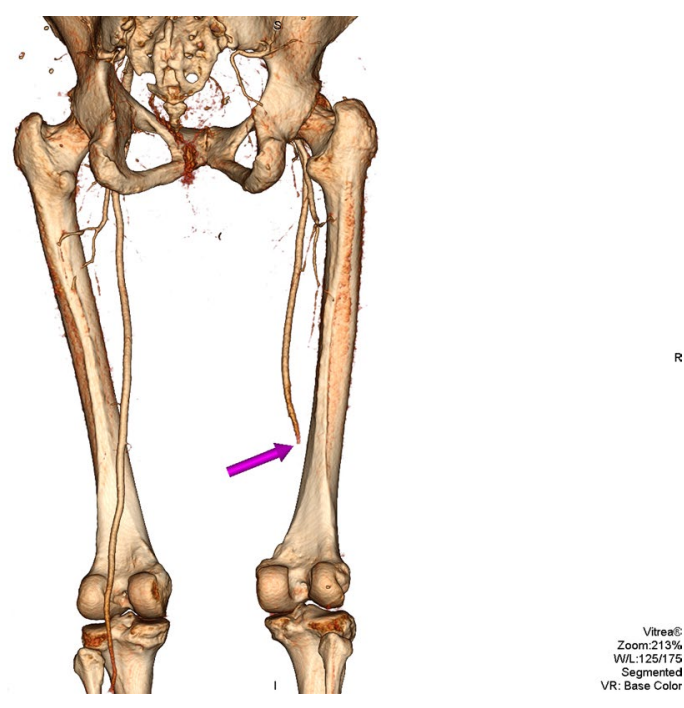


\section{Image 3}

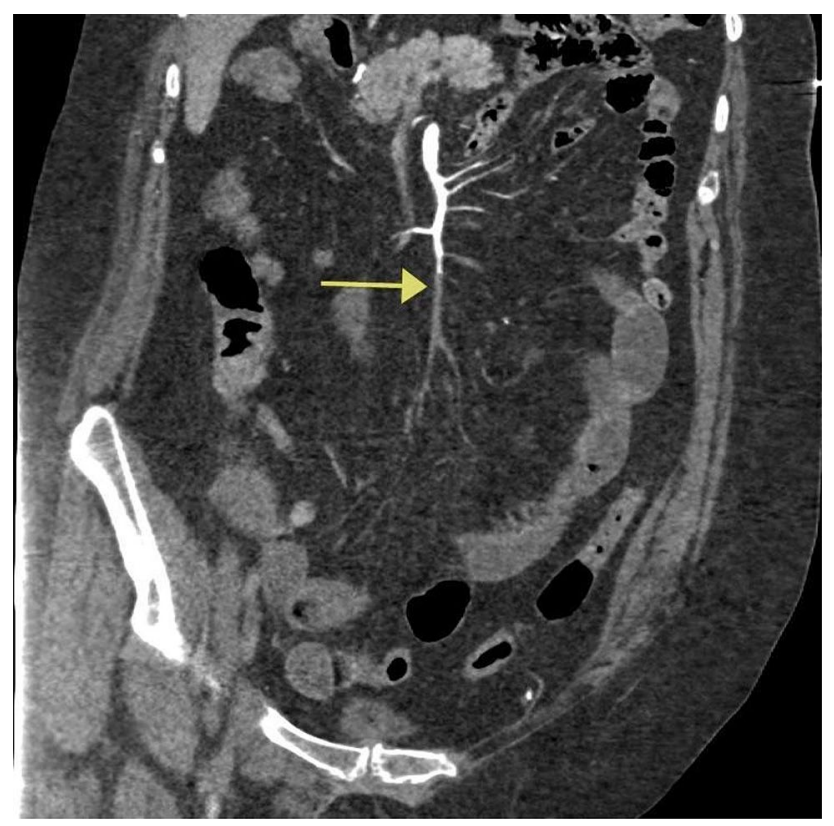

Image 4

Presenter's first name and last name

Mariia Tregubova

Presenter's city and country

Kyiv, Ukraine

Presenter's email address

mariia.tregubova@gmail.com 


\section{9}

\section{Congenital coronary artery fistulas in adults detected by coronary computed tomography angiography}

Anna Michałowska, Pawel Tyczynski, llona Michalowska, Jaroslaw Skowronski, Rafal Wolny, Ilona Kowalik, Cezary Kepka, Mariusz Kruk, Adam Witkowski, Jerzy Pregowski

Keywords Coronary arteries, Coronary artery fistulas, Computed tomography

\section{Topic Area of Interest}

Purpose/Objectives To assess the prevalence and anatomic characteristics of congenital coronary artery fistulas (CAFs) diagnosed with coronary computed tomography angiography (CTA) in adult population.

Methods and Materials Between February 2008 and November 2021 there were 45,817 coronary CTA examinations performed in 39,066 subjects in a single high volume hospital in Poland. The electronic database of all coronary CTA reports was manually screened with use of specific key words to identify consecutive patients with CAF. Following anatomical features were evaluated for all CAFs: size, site of origin and termination, CAF tortuosity and complexity, and presence of atherosclerosis within coronary tree.

Results Forty-two patients with $56 \mathrm{CAFs}$ were identified, $47.6 \%$ were men. The prevalence of CAFs was $0.1 \%$ $(42 / 39,066)$. There were 10 large CAFs (defined as maximal lumen diameter $>10 \mathrm{~mm}$ ). The majority of CAFs arose from the right coronary artery (27/56). The most common site of CAF termination was pulmonary artery (PA) $(33 / 56)$. The site of origin did not have any impact on the size and morphology of CAFs. CAFs terminating in PA and pulmonary arteries were often multiple, bilateral, complex and tortuous ( $\mathrm{p}=0.001, \mathrm{p}<0.001, \mathrm{p}<0.001, \mathrm{p}=0.01$, respectively). CAFs draining into the right structures of the heart were significantly larger at the site of origin and termination compared with CAFs draining into PA and pulmonary arteries as well as into the left structures of the heart $(p=0.024$, $\mathrm{p}=0.035$, respectively). In 22 patients $(52,4 \%)$ atherosclerosis in coronary arteries was found. Patients with stenosis $\geq 50 \%$ within coronary tree $(12 / 22)$ had significantly smaller CAFs $(\mathrm{p}=0.021)$.

Conclusion The most common site of origin and termination of CAFs are RCA and PA, respectively. CAFs draining into PA are more often multiple, complex and tortuous. Significant atherosclerotic changes are often seen in patients with smaller CAFs.
Presenter's first name and last name

Anna Michałowska

Presenter's city and country

Warsaw, Poland

Presenter's email address

an.maria.michalowska@gmail.com

\section{3}

\section{Cardiac CT for validation of clinical and biochemical predictors of coronary artery disease in familial hypercholesterolemia}

\author{
Federica Catapano, Nicola Galea, Livia Marchitelli, Lorenzo \\ Dominici, Francesco Cilia, Camilla Roberti,
}

Marco Francone,

Carlo Catalano

Keywords Coronary arteries, CT/CT-Angiography, Atherosclerosis

\section{Topic Special Focus}

Purpose/Objectives Familial hypercholesterolemia (FH) is an autosomal dominant genetic disorder characterized by a high risk of premature coronary artery disease (CAD). Current cardiovascular disease risk assessment tools are unable to successfully predict long-term cardiovascular risk in FH patients. Cardiac computed tomography angiography (CCTA) may be a key tool to assess subclinical coronary atherosclerosis in this group of patients. The aim of our study is to use CCTA to investigate the presence and extent of CAD in a cohort of patients with FH to retrospectively evaluate clinical and laboratory parameters that can successfully predict disease severity in this population.

Methods and Materials From October 2013 to May 2020 we evaluated 142 consecutive subjects $\geq 18$ years $(49 \pm 12.9$ years, 83 males [58.5\%]) with clinical and genetic diagnosis of FH. Calculation of lipid panel and Dutch Lipid Clinic Network (DLCN) score was performed for each patient. Coronary artery calcium scoring and stenosis degree analysis was performed for all 17 coronary segments. Several atherosclerotic burden CT scores (Agatston score, plaque composition sum (PCS), segment stenosis score (SSS), segment involvement score (SIS)) and CAD-RADS score were calculated. Linear and logistic regressions were used to implement the predictive models.

Results Mean DLCN score was 13 after genotyping. Prevalence of significant CAD (CAD-RADS $\geq 3$ ) was $22.4 \%$ 
(31/138).Independent predictors of SSS were age $(p<0.01)$, gender $(p=0.019)$, monogenic disease $(p=0.043)$, type 2 diabetes mellitus $(p<0.01)$ and the worst recorded triglycerides (TG) level $(p<0.01)$. Independent predictors of SIS were age $(p<0.01)$, type 2 diabetes mellitus $(p=0.013)$, HDL-C $(p=0.011)$ and the duration of LLT $(p<0.01)$. Independent predictors of PCS were age $(p<0.01)$, gender $(p=0.013)$, type 2 diabetes mellitus $(p=0.013)$, HDL-C $(p=0.014)$ and the worst recorded TG level $(p=0.038)$. Independent predictors of Agatston score were age $(p=0.01)$, type 2 diabetes mellitus $(p=0.023)$ and duration of LLT (B: $0.83 ; p=0.033)$. Only age $(p<0.01)$ significantly contributed to prediction of CAD-RADS.

Conclusion Neither lipid panel nor DLCN score can predict presence of significant CAD at CCTA in FH patients. We identified different clinical predictors of CAD severity for different atherosclerotic burden scores, emphasizing the need for a specific risk score for patients with $\mathrm{FH}$, to implement the selection strategy of those patients who should undergo early CCTA, with obvious implications in patienttailored preventive and therapeutic strategies.

Presenter's first name and last name

Federica Catapano

Presenter's city and country

Rome, Italy

Presenter's email address

federica.catapano@uniroma1.it

\section{6}

\section{Effects of the angle on the artifacts related to ICD electrode in dual-energy CT - phantom study}

\section{Piotr Tarkowski, Elżbieta Siek, Grzegorz Staśkiewicz, Andrzej Wójtowicz, Elżbieta Czekajska-Chehab}

Keywords dual-energy, cardiac ct, artifacts

Topic Imaging Technique

Purpose/Objectives The aim of the study was to evaluate changes in size of artifacts related with implemented cardiac devices electrode in phantom study with different angle of the wire in relation to plane of imaging.

Methods and Materials We scanned ICD electrode placed in 3D-prined frame using 64-row single source rapid-kVpswitching dual-energy CT scanner - Revolution GSI (GE Healthcare, Milwaukee, WI). Electrode was scanned in three planes in 5 degrease interval. As reference we used an image acquired with standard helical cardiac gated protocol. The length of two biggest artifacts were measured by two authors independently on monoenergetic reconstruction in $10 \mathrm{keV}$ interval using GSI Volume Viewer (GE Healthcare, Milwaukee, WI) and on the helical image.

Results Reducing the angle of the electrode with respect to the scanning plane resulted in a reduction in the size of the artifacts associated with the electrode tip. The use of monoenergetic reconstructions significantly reduced the size of artifacts in each plane. The largest artifacts were observed when the electrode was in the scanning plane.

Conclusion The greatest reduction of artefacts cause by metal in ICD electrode was possible by decreasing the angle between electrode and plane of scanning. High-energy monoenergetic reconstruction also can reduce these artifacts.

Presenter's first name and last name

Piotr Tarkowski

Presenter's city and country

Lublin, Poland

Presenter's email address

piotrtarkowski1@gmail.com

\section{7}

Diagnostic accuracy of coronary computed tomography angiography (CCTA) for the evaluation of obstructive coronary artery disease in patients referred for transcatheter aortic valve implantation (TAVI): a systematic review and meta-analysis

\section{Marco Gatti, Guglielmo Gallone, Francesco Bruno, Vittoria Poggi, Alessandro Serafini, Ovidio De Filippo, Alessandro Depaoli, Riccardo Faletti, Gaetano Maria De Ferrari, Paolo Fonio, Fabrizio D'Ascenzo}

Keywords Aortic Valve Stenosis, CT/CT-Angiography, Transcatheter Aortic Valve Replacement, Coronary Artery Disease

Topic Imaging Technique

Purpose/Objectives TAVI is now the standard procedure for treating patients with severe aortic stenosis who cannot undergo surgical aortic valve replacement [1]. CTA is a standard procedure in the pre-TAVI diagnostic workup $[1,3]$. However, patients undergoing TAVI have a high pretest risk of severe coronary calcifications (about 50\%) [3], the presence of abnormal rhythm that cannot be controlled with beta-blockers is quite common and they cannot be 
given nitrates, thus complicating the evaluation of CAD with CCTA [1]. Therefore, in accordance with current guidelines, ICA is recommended prior to TAVI to evaluate CAD [1]. On the other hand, CCTA might be a tool to evaluate the presence of coronary tree significant stenosis avoiding unnecessary ICA (reducing risk of complication, costs and dose of iodinated contrast medium) [4, 5]. Based on this consideration, the aim of our metanalysis is to evaluate the diagnostic accuracy of CCTA for the evaluation of coronary tree in patients referred for TAVI.

Methods and Materials PubMed/MEDLINE, EMBASE and CENTRAL were searched up to May 1st, 2021. We included studies reporting data on diagnostic accuracy of CCTA in patients with severe aortic stenosis referred for TAVI who underwent during the diagnostic work-up ICA, who served as a reference. QUADAS-2 tool was used to assess the quality of included studies. The primary endpoint was the patient-level accuracy of CCTA to identify obstructive CAD. For the purpose of this analysis, non-evaluable segments were considered positive based on an intention to diagnose approach. Secondary analysis included the evaluation of the accuracy of CCTA for obstructive CAD at the patient-level excluding patients with non-evaluable segments, at the vessel- and at the segment-level. Subgroup analyses according to CT scanner characteristics (i.e. single heartbeat scanner CTs vs. others) were further performed. Any outcome of interest reported by each study will be included and graphically displayed by forest plots and summary ROC curves.

Results A total of 2228 patients were included in the analysis performed at the patient-level considering nonevaluable segments as positive. The pooled sensitivity and specificity for CCTA were 97\%(94-98\%) and 68\%(56-68\%), respectively and the Positive and Negative Likelihood Radio were 3.0(2.1-4.3) and 0.05[0.03-0.09], respectively; with a diagnostic Odds Ratio of 60(30-121). The HSROC has an AUC $=0.96(0.94-0.98)$. To put our results into clinical practice, estimating a disease prevalence of $40 \%$ in a population of 1000 patients, the study of coronary arteries with CCTA prior to TAVI would correctly avoid 409 (95\%CI 335-470) ICAs. A subgroup analysis comparing single heartbeat scanner CTs to others found no difference in sensitivity [0.96\%(91-98\%) vs. $97 \%(94-99 \%) ; p=0.37]$, whereas the specificity of single heartbeat scanner CTs is higher than others [81\%(67-90\%) vs. $58 \%(43-71 \%) ; p<0.0001]$.

Conclusion In conclusion, CCTA has excellent diagnostic accuracy for assessing obstructive CAD in patients referred for TAVI. Routine CCTA of coronary arteries as part of the pre-TAVI work-up would save more than $40 \%$ of ICAs. The use of single-heart beat scanners, which provide greater specificity, can improve such data even further.

\section{References}

Baumgartner H, Falk V, Bax JJ, et al. (2017) 2017 ESC/ EACTS Guidelines for the management of valvular heart disease. Eur Heart J 38:2739-2791. https://doi.org/10. 1093/eurheartj/ehx391

Knuuti J, Wijns W, Saraste A, et al. (2020) 2019 ESC Guidelines for the diagnosis and management of chronic coronary syndromes. Eur Heart J 41:407-477. https://doi. org/10.1093/eurheartj/ehz425

D’Ascenzo F, Conrotto F, Giordana F, et al. (2013) Midterm prognostic value of coronary artery disease in patients undergoing transcatheter aortic valve implantation: a meta-analysis of adjusted observational results. Int J Cardiol 168:2528-2532. https://doi.org/10.1016/j.ijcard. 2013.03.062

Usman MS, Rawasia WF, Siddiqi TJ, et al. (2019) Metaanalysis Evaluating the Safety and Efficacy of Transcarotid Transcatheter Aortic Valve Implantation. Am J Cardiol 124:1940-1946. https://doi.org/10.1016/j.amjcard.2019. 09.015

Li Y-M, Mei F-Y, Yao Y-J, et al. (2021) Causes and predictors of readmission after transcatheter aortic valve implantation: A meta-analysis and systematic review. Herz 46:1-8. https://doi.org/10.1007/s00059-019-04870-6

\section{Image 1}

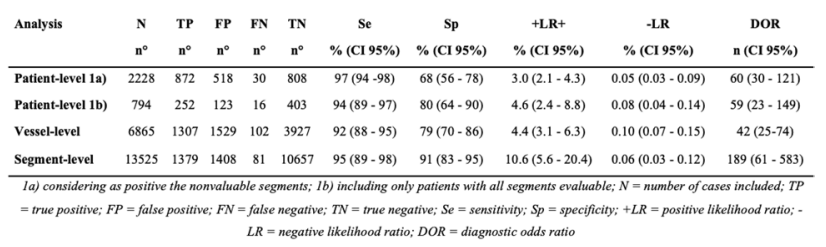

Image 2

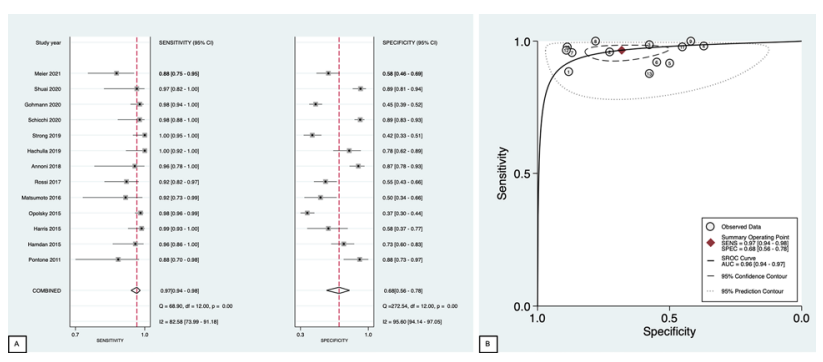




\section{Image 3}

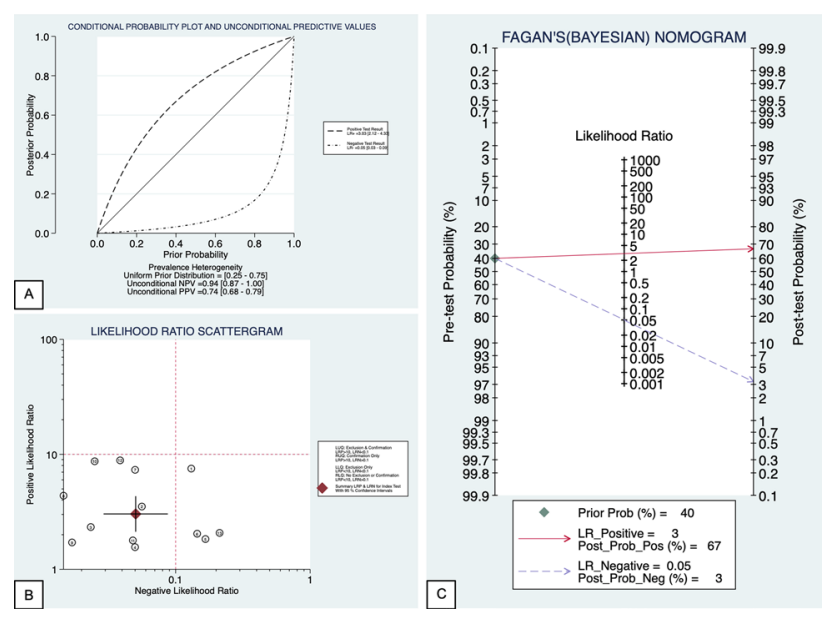

Image 4

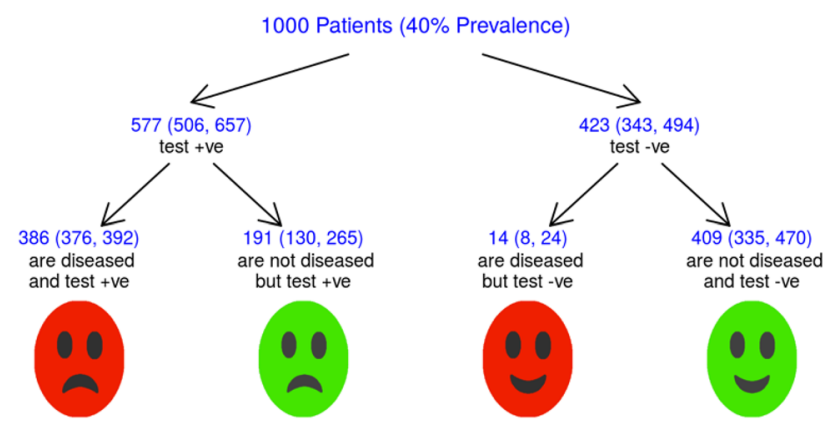

Presenter's first name and last name

Marco Gatti

Presenter's city and country

Turin, Italy

Presenter's email address

marcogatti17@gmail.com

\section{8}

\section{The association of cardiovascular co-morbidities with the hospital mortality in COVID-19 patients \\ Dominik Juskanic, Marek Uhnak, Jana Poláková Mištinová, Lukas Patrovic, Samuel Holly, Miroslav Vytiska}

Keywords Cardiovascular, COVID-19, Hospital mortality

Topic Special Focus

Purpose/Objectives The coronavirus pandemic has changed the structure of in-hospital mortality. The most widely used hospital mortality model (2) considers only the number of co-morbidities. Our objective was to evaluate the specific role of cardiovascular co-morbidities on mortality in COVID-19 patients admitted to hospital and how it performed among others known risk factors in the multivariable mortality prediction model.

Methods and Materials We have retrospectively collected data of 465 patients admitted to the hospital with confirmed COVID-19 by antigen or PCR test. We collected variables of interest from medical records - age, BMI, sex, the presence of diabetes mellitus (DM), cardiovascular and pulmonary co-morbidities. The extent of lung involvement was evaluated on high-resolution computed tomography. For statistical analysis, we used the BESHstat plugin in MS Excel. For categorical variables, we performed the chi-square test and for continuous variables paired t-test. We used a multivariable logistic regression tool for multivariate analysis with mortality as the primary outcome.

Results Multivariable logistic regression analysis revealed, that the most significant influence on mortality was the presence of cardiovascular co-morbidities (OR 3,1, p 0,02, 95\% CI 1,2-8,2) and pulmonary co-morbidities (OR 3,3, $\mathrm{p}<0,0001,95 \%$ CI 1,7-6,5). These were followed by age (OR 1,93, p <0,0001, 95\% CI 1,46-2,55) and extent of pulmonary injury (OR 1,35, p <0,0001, 95\% CI 1,2-1,5). The overall model fit was very good-ROC Wilcoxon AUC 0,85, $\mathrm{p}<0,0001,95 \%$ CI 0,81-0,89).

Conclusion Our model showed that cardiovascular and pulmonary co-morbidities are among the most critical mortality predictors of COVID-19 patients with moderate-to-severe symptoms that require admission to the hospital.

\section{References}

Stewart K, Choudry MI, Buckingham R. Learning from hospital mortality. Clin Med (Lond). 2016;16(6):530-534. https://doi.org/10.7861/clinmedicine.16-6-530

Knight S., Ho A., Pius R. Risk stratification of patients admitted to hospital with covid-19 using the ISARIC WHO 
Clinical Characterisation Protocol: development and validation of the 4C Mortality Score. BMJ 2020; 370: bmj.m3339

Presenter's first name and last name

Dominik Juskanic

Presenter's city and country

Nitra, Slovakia

Presenter's email address

juskanic@gmail.com

\section{0}

\section{Four-dimensional phase contrast MRI with accelerated dual velocity encoding in patients with complex congenital heart disease}

\section{Laurette Kalifa, Charles Roux, Daniel Sidi, Young-wouk Kim, Fraj Bouhajja, Pasquet caroline, Elodie Gouverneur, Véronique Marteau, Marc ZINS, Arshid Azarine}

Keywords dual velocity encoding 4D flow MRI, fourdimensional phase contrast MRI, congenital heart disease

Topic Imaging Technique

Purpose/Objectives To test the feasibility of a dual-velocity encoding (Dual-Venc) 4D Flow MR sequence prototype accelerated by Kt-ARC to assess various vascular flows useful for the follow-up of patients with complex congenital heart disease.

Methods and Materials Routine cardiac MRI was performed in 15 young adults followed for complex congenital heart disease on a $3 \mathrm{~T}$ Magnet (Discovery MR 750, GEMS). The usual 4D flow sequence has been replaced by a Kt-ARC accelerated 4D flow prototype sequence, using two different velocity encoding (Venc) with HighVenc / Low-Venc set to $300 / 100 \mathrm{~cm} / \mathrm{s}$; temporal / spatial resolution $=40-45 \mathrm{~ms} / 2 \times 2 \times 2.2 \mathrm{~mm} 3$, after injection of $0.15 \mathrm{mmol} / \mathrm{kg}$ of a gadolinium-based contrast agent (Gadovist, Bayer, Germany). MRI data was anonymized and sent to a cloud-based software (Arterys). After automatic phase offsets and background correction, a simultaneous analysis of the two separate 4D flow volumes, at high and low Venc, was performed. Velocity-to-noise ratio (VNR), peak velocity and Forward flow were compared between high and low Venc measurements for different vessels: superior and inferior vena cava, pulmonary veins, aorta, portal and hepatic veins. All patients were informed and signed a consent to test the prototype sequence.
Results All dual-venc 4D flow MRIs were successfully acquired in a mean scan time of $18 \pm 3$ min, reducing total scantime by $25 \%$ compared to two separate acquisitions. Cloud-based data post-processing enabled simultaneous real-time analysis of the two 4D-flow datasets at low and high Venc. VNR at low Venc was always higher compared to high Venc (at least twofold). Comparing flow measurements at High and Low Venc, Pearson Correlation coefficients ranged between 0.85-0.95 demonstrating excellent correlation for great vessels (inferior and superior vena cava), but correlation was lower $(0.65-0.75)$ for hepatic and portal veins with lower peak Velocities. An aliasing artifact occurred in most of aortic arterial measurements performed at low Venc.

Conclusion Kt-Arc accelerated Dual-Venc 4D Flow MR sequence is particularly well suited for the follow-up of patients with complex congenital heart disease with challenging flows to measure, as it enables optimal assessment of a wide dynamic velocity range of flows, avoiding the risk of aliasing for high-velocity flows while ensuring a good velocity-to-noise ratio (VNR) for low velocity flows.

Presenter's first name and last name

Laurette KALIFA

Presenter's city and country

PARIS, FRANCE

Presenter's email address

kalifalaurette@hotmail.fr

\section{1}

\section{Evaluation of Left Ventricular Myocardial Global Longitudinal Strain with Cardiac Magnetic Resonance in Hypertrophic CardioMyopathy and correlation with Speckle Tracking Echocardiography}

\section{Vitaliano Buffa, Matteo Macchia, Manuela Madau, Elisabetta Zachara, Federica Re}

Keywords MR/MR-Angiography, Echocardiography/ Echocardiography (transoesophageal), Ultrasound/Ultrasound-Colour Doppler/Ultrasound-Power Doppler

Topic Imaging Technique

Purpose/Objectives Left ventricular (LV) global longitudinal strain 2D Speckle Tracking (2D-GLS Echo-ST) has well accepted for diagnosis and prognosis in Hypertrophic CardioMyopathy (HCM). Cardiac Magnetic Resonance 
Feature Tracking (CMR-FT) has been recognized as an emerging method for the assessment of myocardial deformation in various disorders. Aim of this study was analyze values of GLS, analyze correlation with LV-myocardial mass and compare LV-GLS values from CMR-FT and Echo-ST in patients with HCM.

Methods and Materials CMR of 48 HCM subjects (male, $\mathrm{n}=34$ ) and 30 healthy (male, $\mathrm{n}=13$ ) retrospectively analyzed with a commercial software to assess LV-GLS from short and long axis images 2, 3, 4 chamber views. The LV-GLS of the HCM-group was evaluated also by EchoST. Analysis of continuous variables, ROC curve, linear regression and Bland-Altman plot were performed.

Results LV-GLS CMR-FT was significantly impaired in HCM group vs control $(-14.1 \pm 3.3 \%$ vs $20.9 \pm 1.9 \%$, $\mathrm{p}<0.001$ ), with not significant sex-related difference (males $-14.1 \pm 3.6 \%$, females $14.2 \pm 3.6, \mathrm{p}=0.92$ ) unlike controls (males $-19.8 \pm 1.7 \%$, females $-21.7 \pm 1.7 \%$, $\mathrm{p}=0,006$ ). AUC of ROC curve in diagnosis of HCM was 0.98 with value of $-18.06 \%$ as optimal cut-off. Good correlation was found with myocardial mass $(\mathrm{r}=0.81)$ and fibrosis $(r=0.77)$. Correlation with Echo-ST was good $(r=0.64)$ but with different values (2D-GLS $-15.7 \pm 2.9 \%$, $\mathrm{p}<0,001)$.

Conclusion Myocardial LV-GLS from CMR-FT is significantly impaired (higher) and not sex-related in HCM patients with a diagnostic cut-off of $-18.06 \%$, and correlates with myocardial mass and presence of fibrosis. Interobserver agreement between CMR-FT and Echo_ST was good, even if with a little bias.

Presenter's first name and last name

Vitaliano Buffa

Presenter's city and country

Roma Italy

Presenter's email address

vitalianobuffa@libero.it

\section{2}

\section{Role of Gemstone Spectral Imaging in Evaluation of Coronary Artery Disease: A Primer for Cardiac Radiologists}

\author{
Smily Sharma, VENKATA SUBBAIH ARUNACHALAM, Rakesh \\ Chauhan
}

Keywords CT/CT-Angiography, Dual Energy CT, Plaque Imaging, Myocardial Iodine Quantification
Topic Imaging Technique

Purpose/Objectives To study role of Gemstone Spectral Imaging (GSI) in evaluation of Coronary Artery Disease. To study principles of evaluation of characteristics of Coronary artery plaque using GSI. To study role of GSI in myocardial perfusion analysis.

Methods and Materials Using GE Revolution Frontier 256 Slice Single Source Dual Energy CT available in one of the author's institution, detailed evaluation of coronary artery disease was done by prospective Cardiac Gated CT Coronary Angiography. $70 \mathrm{keV}$ Monochromatic images were used for interpretation. In addition to detailed analysis of coronary arteries, Coronary Artery Plaques were evaluated by their charcteristic Spectral HU curves and Effective Z (atomic) numbers. Myocardial Iodine Quantification was done to look for myocardial perfusion defects.

Results Monochromatic images obtained with Gemstone Spectral Imaging helped in improving the detection of plaques in coronary arteries with better Contrast to Noise Ratio. Plaque analysis with spectral HU curves helped to differentiate fatty, calcified and fibrous plaques by their specific attenuation characteristics at multiple monoenergetic levels. The calculation of effective atomic numbers of the plaque and their comparison with Effective $\mathrm{Z}$ of materials like fat, fibrous tissue and calcium further added confirmation to the characteristic of the plaque. By estimation of iodine concentration in the myocardium, perfusion defects were identified which corresponded to areas of low iodine uptake.

Conclusion Gemstone Spectral Imaging has revolutionalized the evaluation of coronary artery disease. Benefits like evaluation of characteristics of plaques and quantification of functional myocardial tissue are instrumental in deciding the treatment strategy and guiding the prognosis of the patient.

\section{References}

Quantitative myocardial perfusion with stress dual-energy CT: Iodine concentration differences between normal and ischemic or necrotic myocardium. Initial experience Carlos Delgado, Eur Radiol, 2015. https://doi.org/10.1007/ s00330-015-4128-y.

Evaluation of image quality of coronary artery plaque with rapid kvp-switching dual energy CT. Y Ohta et al. Clinical Imaging 43 (2017) 42-49.

\section{Image 1}


Jodhpur, India

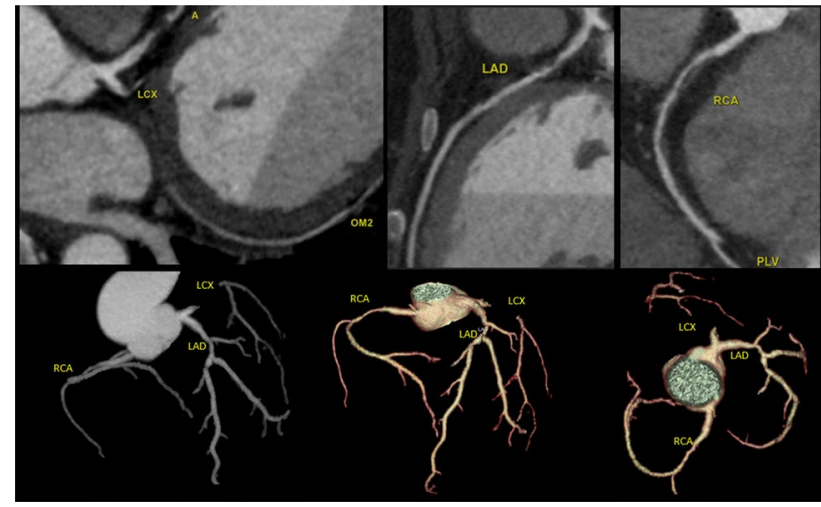

Presenter's email address

drsmilysharma@gmail.com,smilysharma1592@gmail.com

\section{3}

\section{Diagnostic value of CT-based calculation of fractional flow reserve (CTFFR) in CAD patients compared to interventional fractional flow reserve: feasibility and clinical results on a 128-slice computed tomography}

\section{Image 2}

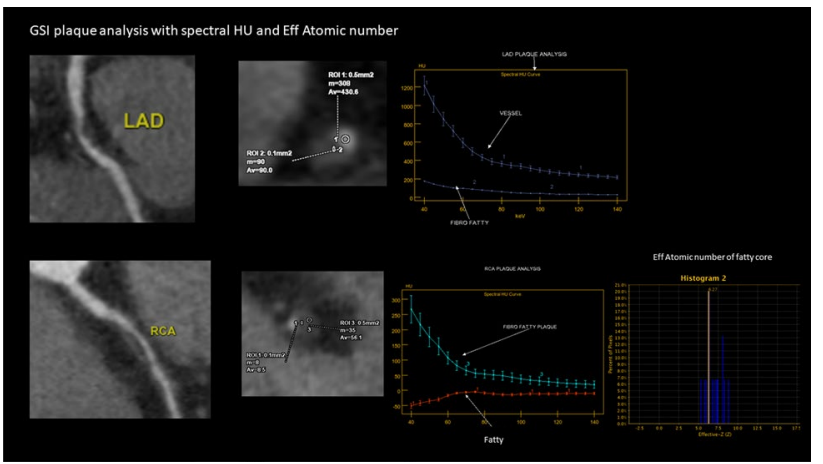

Christopher Kloth, Theresia Baumeister, Steffen Klömpken, Dominik Buckert, Peter Bernhardt, Christoph Panknin, Meinrad Beer, Horst Brunner

Keywords CT/CT-Angiography, CAD, FFR

Topic Imaging Technique

Purpose/Objectives Feasibility approach and comparison of diagnostic accuracy of fractional flow reserve from coronary computed tomographic angiography [CTFFR] acquired on a 128 slice scanner against diagnostic accuracy of coronary computed tomographic angiography (CCTA), invasive catheter angiography (CA) and invasive fractional flow reserve [IFFR].

Methods and Materials This single centre retrospective analysis included 270 patients ( 80 female; mean age $63.80 \pm 10.39$ years) with CCTA within 21 months. CTFFR

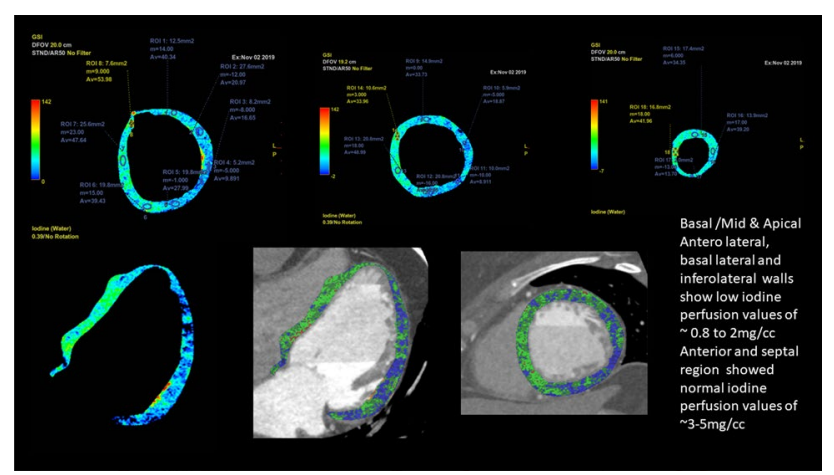
values were obtained using a deep learning [DL] based non-commercial research prototype (cFFR Version 3.2.0; Siemens Healthineers), which allows on site CTFFR computation with minimal interaction. The measurements were compared with invasive coronary angiography in 87/270 patients and with invasive fractional flow reserve measurement in $5 / 87$ patients of these.

Results A total of 539 stenoses were detected (129 onevessel, 73 two-vessel and 68 with three-vessel CAD) in CCTA; of these, $81 / 539$ (13.9\%) were classified as mild, $327 / 539(61.8 \%)$ as moderate and $131 / 539(24.3 \%)$ as highgrade. Non-invasive CTFFR has so far been evaluated in $75 / 270$ patients $(27.7 \%)$. The mean non-invasive CTFFR was 0.93 (range $0.33-0.99$ ). In a total of 9 patients (3.3\%), a relevant reduction in CTFFR below 0.75 was found. Per vessel sensitivity, specificity, PPV, NPV and accuracy of CTFFR were 0.63, 0.99, 0.94, 94.4 and 0.94, compared to

Presenter's first name and last name Smily Sharma

Presenter's city and country invasive coronary angiography. Per vessel sensitivity, specificity, PPV, NPV and accuracy of CCTA were 0.92, 0.96, $0.91,0.96$ and 0.95 . The mean CTFFR processing time was $10.13 \mathrm{~min} \pm 2.83 \mathrm{~min}$. 
Conclusion Non-invasive onsite quantification of CTFFR is feasible with minimal observer interaction in a routine real-world setting (consecutive patients with intermediate CAD risks scanned on a 128slice scanner without exclusion criteria for CTFFR). Functional information obtained from CTFFR might be promising especially under new CAD guidelines. Innovative/novel DL based algorithms allow a robust and semi-automatic determination of CTFFR on data from standard CT scanners.

\section{Image 1}

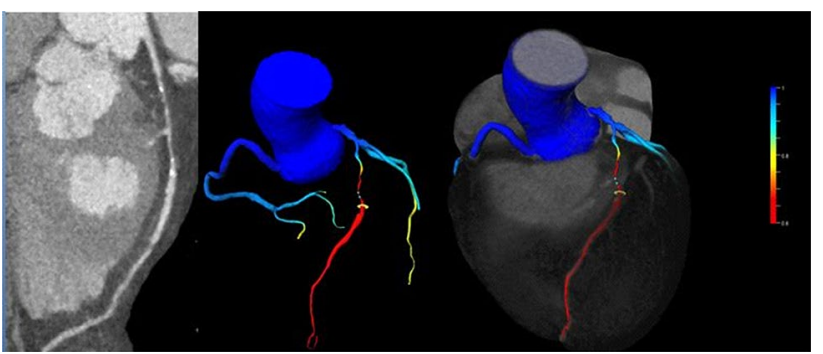

Presenter's first name and last name

Christopher Kloth

Presenter's city and country

Ulm-Germany

Presenter's email address

christopher.kloth@uniklinik-ulm.de

\section{5}

\section{Prevalence and Prognostic value of Subclinical Coronary Artery Disease detected with CT for left atrium and pulmonary veins In Patients undergoing Atrial Fibrillation ablation (PROCADAF Study): preliminary results}

\section{Andrea Daniele Annoni, Eleonora Carlicchi, Daniele Andreini, Alberto Formenti, Giuseppe Muscogiuri, Maria Elisabetta Mancini, Maria Antonietta Dessanai, Saima Mushtaq, Andrea Baggiano, Gianluca Pontone}

Keywords CT/CT-Angiography, atrial fibrillation, coronary artery disease, ablation procedure

Topic Imaging Technique
Purpose/Objectives Atrial fibrillation (AF) is the most common sustained cardiac arrhythmia. Clinical predictors such as persistent $\mathrm{AF}$, increased left atrial volume and metabolic syndrome have been demonstrated to be associated with $\mathrm{AF}$ recurrence after ablation procedure (ATC). However, the impact of coronary heart disease (especially subclinical) and revascularization procedures on the outcome of rhythm control after ATC has not been extensively investigated. Therefore, the purpose of the study is to retrospectively evaluate the incidence of subclinical CAD in a population of patients with Atrial Fibrillation, that underwent $\mathrm{CT}$ examination for left atrium and pulmonary veins assessment for ablation procedure planning.

Methods and Materials 173 patients that underwent CT for left atrium and pulmonary veins for ablation procedure planning with post procedure clinical follow up were enrolled in the study. Patients were divided into 2 groups according to the history of coronary artery disease (CAD) (Group 1: known CAD / previous revascularization procedure and Group 2: no history of CAD). CT exams of Group 2 patients were retrospectively evaluated to assess the presence of subclinical CAD. The presence of atherosclerotic plaques was recorded for each coronary segment (according to the AHA 16 segments classification). No quantification of coronary stenosis was performed. The correlation between clinical / procedural outcome and AF recurrence in the two groups during a mean follow up time of about 3 years was compared.

Results 51 patients had known history of CAD (Group 1). 122 pts out of 173 had no history of CAD (Group 2). In group $2 \mathrm{CT}$ revision showed the presence of subclinical CAD in 71 pts (58\%). Overall AF recurrency after ATC was recorded in 17 out of 51 patients of group 1 ( $33 \%$ ) while in group 2 AF recurrency was observed in 58 out of 122 patients (47\%). 13 patients with CT evidence of CAD had no AF recurrency. Kaplan-Meier curves showed an higher AF recurrency rate in patients with subclinical CAD during a mean follow up period of 730 days (2 years) while not significant recurrency rate was observed in longer follow up with slighty higher recurrency rate in patients with known history of CAD.

Conclusion In conclusion, in patients without known history of CAD, prevalence of subclinical CAD was higher in those with AF recurrency and associated with less efficacy of ablation procedure than those with sinus rhythm during follow up. To identify subclinical CAD before ATC has potential clinical indications for cardiac arrhythmia management.

Presenter's first name and last name Eleonora Carlicchi

Presenter's city and country

Milan, Italy 
Presenter's email address

eleonora.carlicchi@gmail.com

\section{6}

\section{Optimization of preprocedural ECG-gated full-cycle-CT in patients referred for transcatheter mitral or tricuspid valve repair}

\section{Felix Escher, Adrian Curta}

Keywords Bolus Tracking, Test Bolus, ECG-gated, FullCycle-CT, CT/CT-Angiography, Transcatheter Mitral Valve Repair, Transcatheter Tricuspid Valve Repair, Preinterventional Planning, Optimization, Heart Failure

\section{Topic Imaging Technique}

Purpose/Objectives Advancements in transcatheter mitral and tricuspid valve repair have resulted in growing demands for pre-procedural imaging. Multidetector ECG-gated full cycle CT (MDCT) plays an increasing role for interventional planning in these patients as it is useful in annular evaluation and landing zone characterization. As both mitral and tricuspid valves represent complex anatomical structures good image quality is key. With the introduction of MDCT and shorter acquisition times, optimal synchronization between the passage of contrast agent and data acquisition is mandatory. Additionally, heart failure and arrhythmias are common in these patients posing further challenges for CT imaging. Thus, precise acquisition timing is crucial for optimal contrast enhancement. There is no consensus on which acquisition technique should be used. We aimed to optimize our pre-procedural CT protocol comparing bolus tracking (BT) and test bolus (TB) techniques. To our knowledge, there is no publication that has examined these techniques in this patient population.

Methods and Materials We performed a retrospective analysis on $n=157$ pre-procedural ECG-gated full-cycle CT scans (BT group $n=70$; TB group $n=87$ ) after a protocol change was made from BT to TB technique. Contrast-tonoise (CNR) ratios were obtained by dividing density adjacent to the mitral or tricuspid valve by the density of air next to the patient. Demographic data, laboratory, ECG and TTE/TEE parameters were collected from electronic health records. Also, volume of contrast agent and saline chaser as well as radiation dose length product (DLP) and maS were collected. Between-group comparisons were performed using a standard T-test, Mann-Whitney-U-Test or Fisher's Exact test, as appropriate.

Results BT and TB resulted in comparable CNR (BT: 0.47 (0.16-0.98); TB: $0.54(0.11-1.40) ; \mathrm{p}=0.1)$. BT was associated with a shorter scan duration (BT: 8.1 (4.1-24.4); TB: $13.8(6.2-41.4) ; \mathrm{p}<0.001)$, less radiation in terms of DLP (BT: $1175 \pm 588$; TB: $1386 \pm 675, \mathrm{p}=0.04$ ) and lower total volume administration (BT: $101 \mathrm{ml}$ (61-161); TB: $114 \mathrm{ml}$ (70-150);p <0.001). In a subset of patients with severely impaired ejection fraction (LVEF $\leq 35 \% ; n=44(\mathrm{~TB} n=26$; BT $n=18)$ using the TB technique yielded significantly better image quality in terms of CNR (TB: $0.55(0.29-1.40)$; BT: $0.43(0.16-0.69)$; $\mathrm{p}=0.02)$.

Conclusion BT showed advantages in terms of shorter duration, less radiation and lower contrast agent volume. In patients with impaired LVEF (LVEF $\leq 35 \%$ ), the TB technique yielded superior image quality and may be the preferred approach in this specific patient population.

\section{References}

Faggioni L, Gabelloni M, Accogli S, Angelillis M, Costa G, Spontoni P, et al. Preprocedural planning of transcatheter mitral valve interventions by multidetector CT: What the radiologist needs to know. Eur J Radiol Open. 2018;5:131-40.

Blanke P, Naoum C, Webb J, Dvir D, Hahn RT, Grayburn P, et al. Multimodality Imaging in the Context of Transcatheter Mitral Valve Replacement: Establishing Consensus Among Modalities and Disciplines. Vol. 8. United States; 2015.

Scholtz J-E, Ghoshhajra B. Advances in cardiac CT contrast injection and acquisition protocols. Cardiovasc Diagn Ther. 2017 Oct;7(5):439-51.

Prokop M. Multislice CT angiography. Eur J Radiol. 2000 Nov;36(2):86-96.

Cademartiri F, Nieman K, van der Lugt A, Raaijmakers RH, Mollet N, Pattynama PMT, et al. Intravenous contrast material administration at 16-detector row helical CT coronary angiography: test bolus versus bolus-tracking technique. Radiology. 2004 Dec;233(3):817-23. 


\section{Image 1}

\begin{tabular}{|c|c|c|c|}
\hline Characteristics & $\begin{array}{c}\text { Bolus Tracking } \\
(\mathrm{n}=70)\end{array}$ & $\begin{array}{c}\text { Test Bolus } \\
(\mathrm{n}=87)\end{array}$ & P Value \\
\hline \multicolumn{4}{|l|}{ Demographic Characteristics } \\
\hline Sex (male), $n(\%)$ & $35(50)$ & $42(48.3)$ & 0.87 \\
\hline Age (median) (range), y & $78.4(32-91)$ & $75.7(42-92)$ & 0.94 \\
\hline BMI (median) (range), $\mathrm{kg} / \mathrm{m}^{2}$ & $24.6(17-35)$ & $24.8(17-40)$ & 0.24 \\
\hline \multicolumn{4}{|l|}{ Renal Characteristics } \\
\hline eGFR $($ mean $\pm \mathrm{SD}) \mathrm{ml} / \mathrm{min}$ & $59.9 \pm 22.6$ & $53.3 \pm 19.4$ & 0.07 \\
\hline \multicolumn{4}{|l|}{ Cardiac Characteristics } \\
\hline Heart Rate (median) (range), bpm & $76(45-103)$ & $73(46-117)$ & 0.26 \\
\hline LV-EF (median) (range), \% & $55.8(20-72)$ & $55.6(19-74)$ & 0.75 \\
\hline NT-pro BNP (median) (range), ng/l & $2247(626-48463)$ & $2070(68-3347)$ & 0.57 \\
\hline Atrial Fibrillation, $n(\%)$ & $42(60)$ & $40(46.0)$ & 0.11 \\
\hline \multirow{2}{*}{\multicolumn{4}{|c|}{$\begin{array}{l}\text { Data from } 157 \text { patients are reported as mean }( \pm \text { standard deviation), median (range) or number } \\
\text { (percentage). BMI = Body Mass Index; NT-proBNP }=N \text {-terminal pro-B-type natriuretic peptide; } L V \text {-EF }\end{array}$}} \\
\hline & & & \\
\hline \multicolumn{4}{|c|}{$=$, Left ventricular ejection fraction } \\
\hline \multicolumn{4}{|c|}{ Table 3 Baseline Characteristics (LVEF $\leq 35 \%$ ) } \\
\hline Characteristics & $\begin{array}{l}\text { Bolus Tracking } \\
\quad(n=18)\end{array}$ & $\begin{array}{c}\text { Test Bolus } \\
(n=26)\end{array}$ & PValue \\
\hline \multicolumn{4}{|l|}{ Demographic Characteristics } \\
\hline Sex (male), $n(\%)$ & $14(77.8)$ & $13(50.0)$ & 0.11 \\
\hline Age (median) (range), y & $72.7(32-84)$ & $74.1(53-83)$ & 0.45 \\
\hline BMI (median) (range), $\mathrm{kg} / \mathrm{m}^{2}$ & $23.4(17-32)$ & $26.0(19-40)$ & 0.13 \\
\hline \multicolumn{4}{|l|}{ Renal Characteristics } \\
\hline eGFR (mean $\pm \mathrm{SD}$ ) $\mathrm{ml} / \mathrm{min}$ & $67.2 \pm 26.3$ & $43.3 \pm 18.1$ & $<0.01$ \\
\hline Creatinine (median) (range), $\mathrm{mg} / \mathrm{dl}$ & $1.2(0.9-4.6)$ & $1.5(0.8-5.1)$ & 0.24 \\
\hline \multicolumn{4}{|l|}{ Cardiac Characteristics } \\
\hline Heart Rate (median) (range), bpm & $78(45-103)$ & $72(49-117)$ & 0.74 \\
\hline LV-EF (median) (range), \% & $33.0(20-35)$ & $34.0(19-35)$ & 0.50 \\
\hline NT-pro BNP (median) (range), ng/l & $4681(626-16968)$ & $4981(737-33472)$ & 0.96 \\
\hline Atrial Fibrillation, $\mathrm{n}(\%)$ & $8(44.4)$ & $8(31.0)$ & 0.53 \\
\hline
\end{tabular}

\section{Image 2}

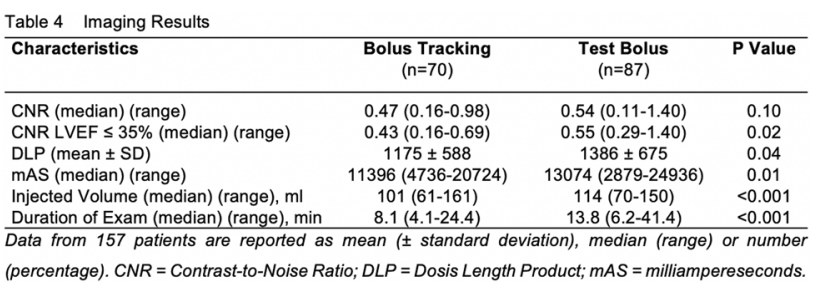

Presenter's first name and last name

Felix Escher

Presenter's city and country

Munich, Germany

Presenter's email address

felix.escher@med.uni-muenchen.de
0077

\section{Criss-cross heart with L-TGA, DORV, Pulmonary Valve Stenosis, ASD, VSD and MAPCAs. Long term CMR assessment with after Fontan surgical repair}

\section{Placido Romeo, Celona Antonio, Farruggio Silvia, Inserra Maria Cristina, Baccano Giovanna, Giambattista Privitera, Agati Salvatore, Calvaruso Davide, Caruso Elio}

Keywords Congenital heart disease, Arteriovenous malformations, Physiology

\section{Topic Special Focus}

Purpose/Objectives Illustrate an extremely rare complex Congenital Heart Disease(CHD) surgically corrected with Fontan intervention assessed with Cardiac Magnetic Resonance(CMR) in a grown-up patient $(\mathrm{GUCH})$, CrissCross Heart $(\mathrm{CCH})$ accounts for about $0,1 \%$ of congenital heart defects and in the context of dextrocardia is even rarer. Its often associated with other congenital malformations like hypoplasia of right ventricle(RV) and Tricuspid Valve(TV), Ventricular Septal Defect(VSD), Transposition of Great Arteries(TGA) or Double Outlet Right Ventricle(DORV) and Pulmonary Stenosis. Alterations of atrio-ventricular (AV) alignment and disharmonies between segmental situs and AV alignment are also possible. Less frequent anomalies are straddling of TV or Mitral Valve(MV), MV stenosis, sub-valvular aortic stenosis or aortic arch obstruction. We report a case of dextrocardia in situs solitus, with AV concordance, DORV, VSD and Atrial Septal Defect (ASD) in a L-Looped TGA, with subpulmunary valvular stenosis. Major Aorto-Pulmonary Collateral Arteries(MAPCAs) were also present in a 32 years old patient after early surgical repair.

Methods and Materials The patient was studied in our Radiologic Department to comprehensively assess his clinical situation with cardiac MRI after an incomplete echocardiographic examination constrained by unsatisfactory acoustic window. CMR was performed with cine balanced steady state free precession (b-SSFP) acquisition in 2,3 and 4-chamber view, Right and Left Ventricle Outlets and Short Axis and 2-chamber stack on Right Ventricle to investigate volumes and ventricular kinesis. Phase contrast (PC) were performed to obtain flows. Contrast Enhanced Magnetic Resonance Angiography (CEMRA) demonstrated vascular patency and 3D Whole Heart acquisitions showed such complex cardiovascular anatomy after Fontan 
surgical repair. Late enhancement was assessed with Phase Sensitive Inversion Recovery (PSIR) sequences. The study was completed with 3D Volume Rendering (VR) and Multiplanar Reconstructions (MPR).

Results The examination demonstrates a quite good ejection fraction $(\mathrm{EF}=54 \%)$, a mild hypertrophy of ventricular wall $(105 \mathrm{~g} / \mathrm{m} 2)$. Occlusion of native pulmonary artery was recognized due to surgical ligation. A good patency of Fontan conduit and pulmonary arteries resulted at CEMRA acquisition. MAPCAs were present, justifying flow discrepancy between Fontan circulation and aorta (QP/QS of 0,8). Late gadolinium enhancement stripe was present on right ventricle anterior wall near aortic outlet. Conclusion Cardiac MRI with image post processing offers a comprehensive tool to explore clinical situation in $\mathrm{CHD}$, avoiding invasive diagnostic angiography to assess flows and QP/QS. 3D image post processing eventually completed with 3D printing helps Heart Team in decision making especially when facing surgical unsatisfactory result or complications.

\section{References}

Manuel D, Ghosh G, Joseph G, Lahiri A, George PV. Criss-cross heart: Transthoracic echocardiographic features. Indian Heart Journal. 2018;70(1):71-4.

Kasar T. Criss-cross heart with dextrocardia and transposition of the great arteries: a rare pathology. Turk Kardiyoloji Dernegi Arsivi-Archives of the Turkish Society of Cardiology. 2015;

Somasundaram A, Phillips M, Provenzano, S. C. J., Ward, C. A pictorial review of the role of 3.0 T MRI in the preand post- operative management of simple and complex paediatric cases of transposition of the great arteries. 2013;

Oliveira ÍM de, Aiello VD, Mindêllo MMA, Martins Y de O, Pinto Jr VC. Criss-cross heart: report of two cases, anatomic and surgical description and literature review. Revista Brasileira de Cirurgia Cardiovascular. 2013;28(1):93-102. Ming Z, Yumin Z. Magnetic Resonance Evaluation of CrissCross Heart. Pediatric Cardiology. 2007;29(2):359_

\section{Image 1}

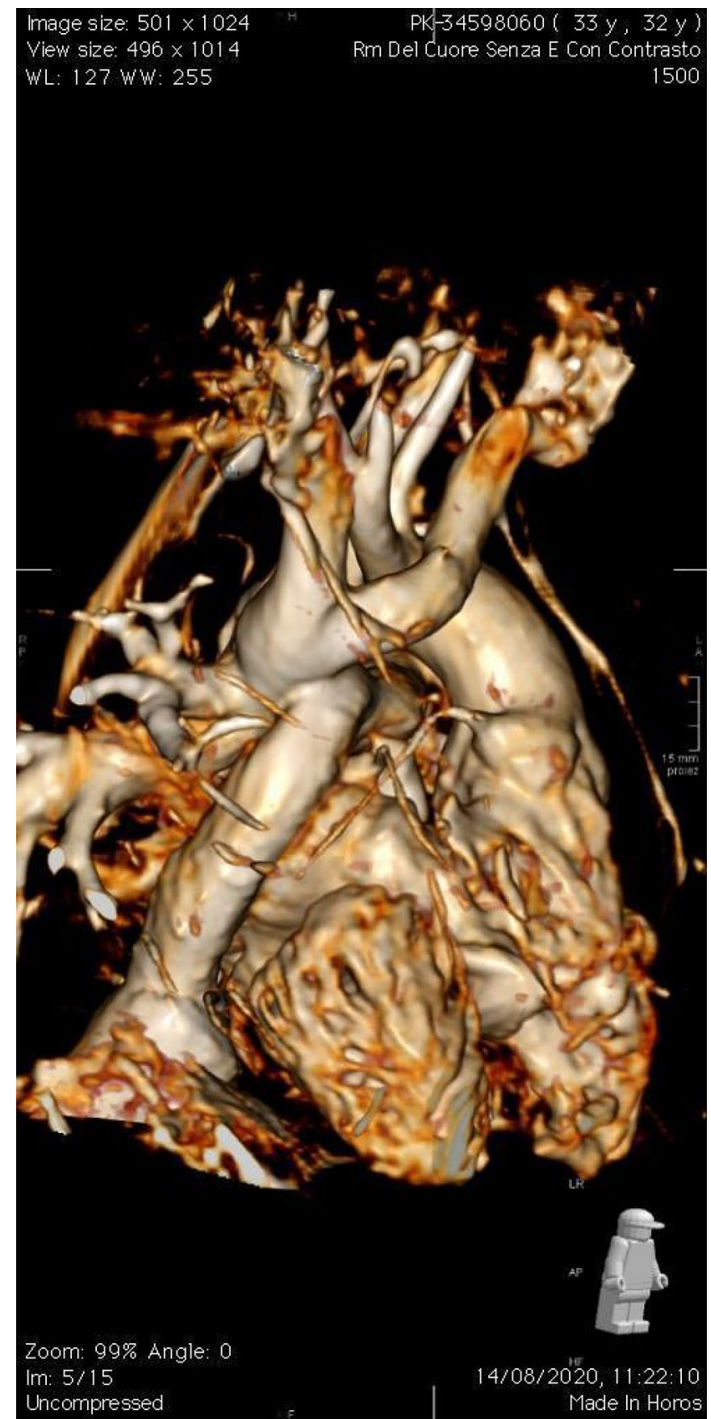




\section{Image 2}

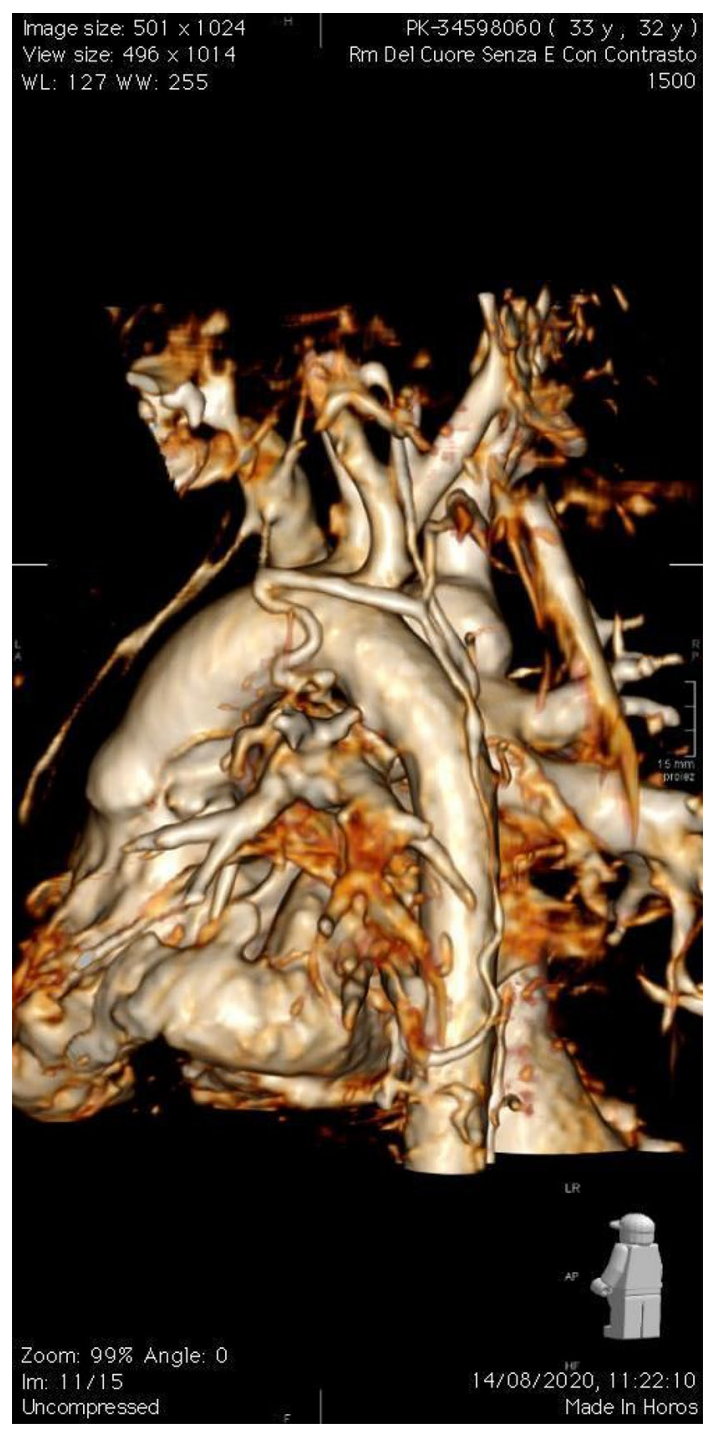

Image 3

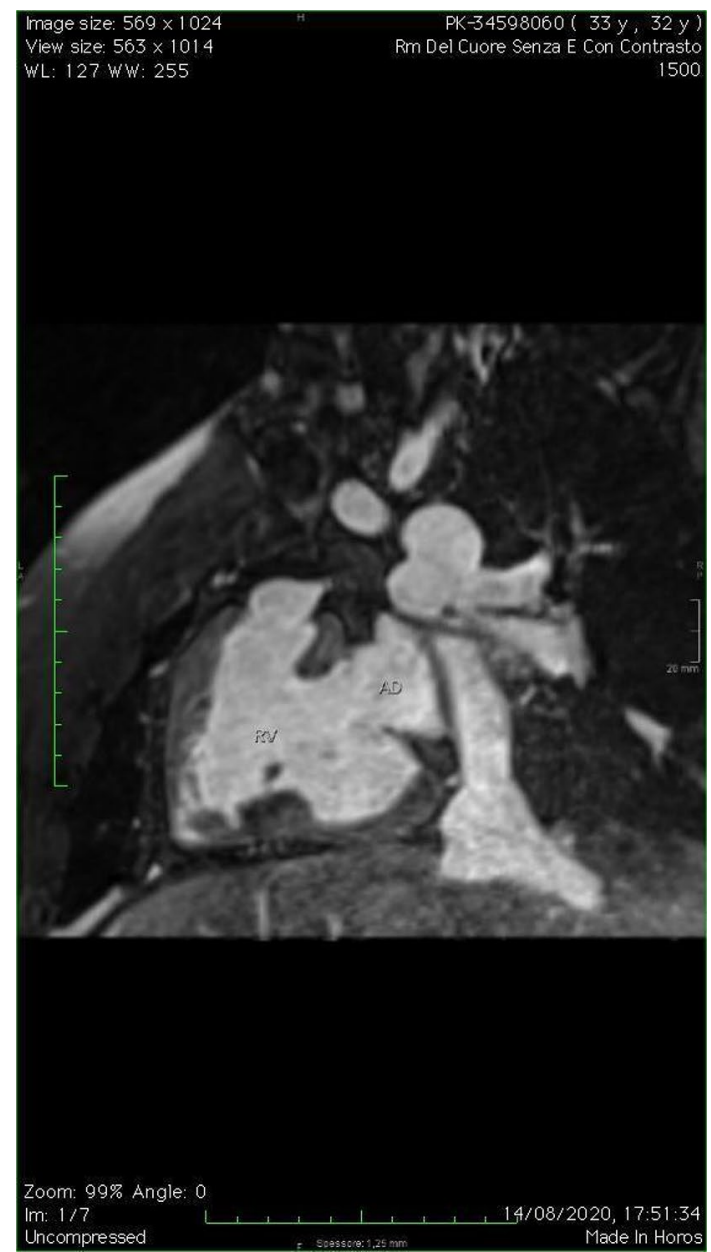




\section{Image 4}

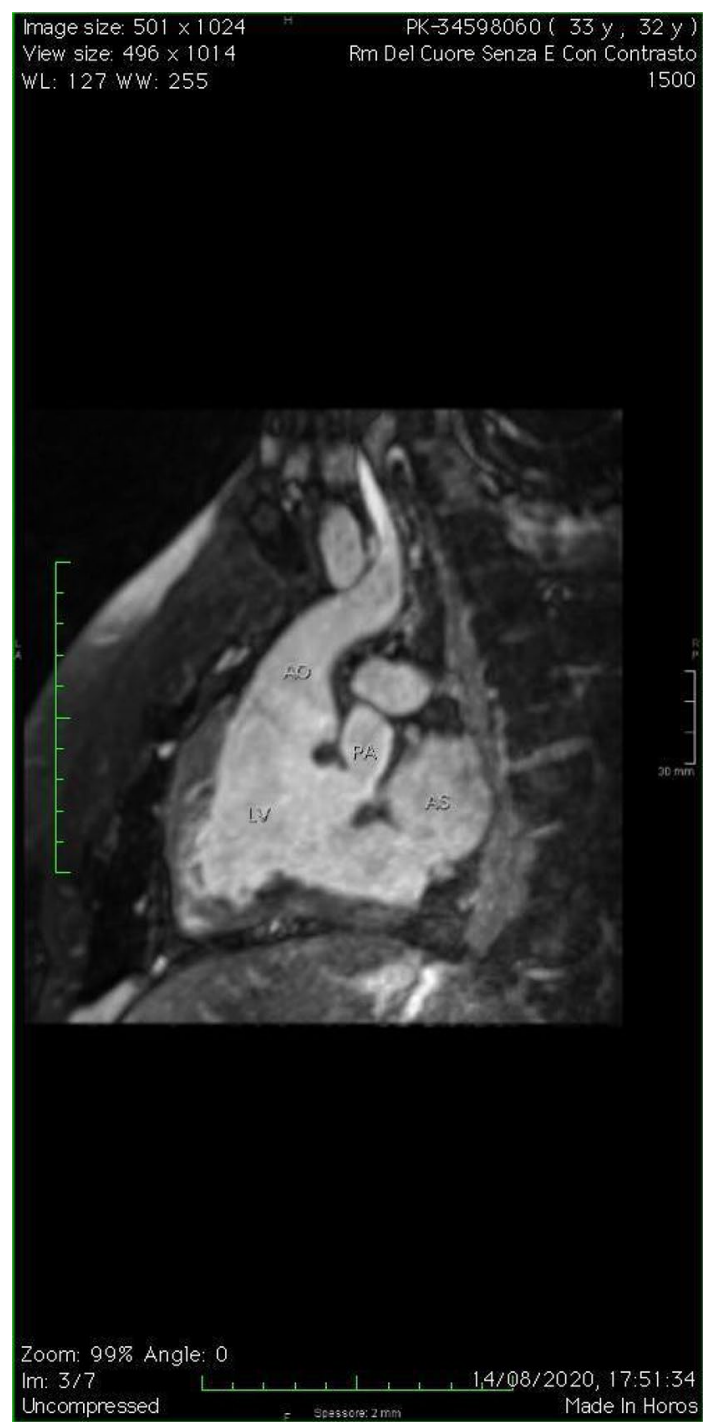

Presenter's first name and last name

Placido Romeo

Presenter's city and country

Taormina, Italy

Presenter's email address

promeo@sirm.org
0080

\section{Role of multiparametric cardiac MR in patients with Antisynthetase syndrome}

\section{Gemma Burcet,, Gemma Burcet, Jose L Reyes-Juarez, Filipa Valente, Albert Selva-O'Callaghan, Albert Gil, Albert Roque, Hug Cuellar-Calabria}

Keywords Cardiac valves, Carotid arteries, Coronary arteries

\section{Topic Area of Interest}

Purpose/Objectives Antisynthetase syndrome (ASS) is an autoimmune disease characterized by inflammatory myopathy, interstitial lung disease, arthritis, mechanical hands and Raynaud phenomenon, among other features. Recent studies have shown that idiopathic inflammatory myopathies (IIM) may develop cardiac involvement, either ischemic (coronary artery disease) or inflammatory (myocarditis). There is an interest in knowing whether patients with the ASS may have silent myocardial interstitial involvement. New cardiac MR (CMR) mapping techniques may detect subclinical myocardial involvement in the absence of visible late gadolinium enhancement (LGE), mainly as edema (increased extracellular volume in the interstitium and extracellular matrix). Therefore, the purpose of this study was to identify alterations in multiparametric cardiac MR in patients with ASS in the acute and chronic stage.

Methods and Materials In an ongoing study starting from 2020, all patients diagnosed with ASS at the myositis unit of the Internal Medicine department undergo a CMR. The patients also received the standard clinical workup, investigation of specific and associated myositis antibodies and high-resolution chest CT (HRCT) for detecting lung and systemic involvement. The CMR protocol includes standard morphologic, functional and LGE sequences in cardiac planes, as well as T1 and T2 mapping sequences with extracellular volume (ECV) calculation. Nineteen participants have been included to this date $(42 \%$ men, mean age $56.6 \pm 11.4$ years). The overall population had a mean disease evolution time from diagnosis of $6.10 \mathrm{y} \pm 7.13$ years (range 2 months to 26 years). Two patients were in an acute stage at the time of inclusion (11\%) and their mean disease evolution time was 4.5 months.

Results Six patients (32\%) had an elevated myocardial T2 value $(>50 \mathrm{~ms})$, associated to elevation of the $\mathrm{T} 1$ value (>1050 ms), increased ECV (>30\%) or both, in 1, 1 and 3 cases, respectively. Patients in the acute stage showed a trend towards elevated T2 values $(p=0.09)$, and had the highest $\mathrm{T} 2$ values (58 and $60 \mathrm{~ms}$, respectively). A mild isolated 
elevation of the $\mathrm{T} 1$ value $(1057 \mathrm{~ms})$ was detected in a single patient in the chronic stage. No patients had segmental contraction alterations or ischemic LGE patterns, although two patients in a chronic stage (11\%) had a mild reduction of left ventricle ejection fraction (both 55\%).

Conclusion In this ongoing study, we present a series of 19 patients with ASS who underwent cardiac MR with mapping sequences. We found subclinical myocardial involvement in patients with ASS in the acute as well as in the chronic stage, most frequently as elevation of myocardial $\mathrm{T} 2$ value with or without elevation of T1 value and ECV. Patients in the acute stage showed a tend towards elevated $\mathrm{T} 2$ values.

\section{References}

Dieval C et al. Myocarditis in Patients With Antisynthetase Syndrome: Prevalence, Presentation, and Outcomes. Medicine (Baltimore). $2015 \mathrm{Jul}$;94(26):e798.
Sharma K, Orbai AM, Desai D, Cingolani OH, Halushka MK, Christopher-Stine L, Mammen AL, Wu KC, Zakaria S. Brief report: antisynthetase syndrome-associated myocarditis. J Card Fail. 2014 Dec;20(12):939-45.

Presenter's first name and last name

Gemma Burcet

Presenter's city and country

Barcelona, Spain

Presenter's email address

gemma.burcet.idi@gencat.cat

Publisher's Note Springer Nature remains neutral with regard to jurisdictional claims in published maps and institutional affiliations. 